\title{
Holography for Schrödinger backgrounds
}

\author{
Monica Guica, ${ }^{a}$ Kostas Skenderis, ${ }^{b, c}$ Marika Taylor ${ }^{b}$ and Balt C. van Rees ${ }^{b}$ \\ ${ }^{a}$ Laboratoire de Physique Théorique et Hautes Energies (LPTHE), \\ CNRS, UPMC, Univ. Paris 6, Boite 126, 4 Place Jussieu, \\ 75252 Paris Cedex 05, France \\ ${ }^{b}$ Institute for Theoretical Physics, \\ Science Park 904, Postbus 94485, 1090 GL Amsterdam, The Netherlands \\ ${ }^{c}$ Korteweg-de Vries Institute for Mathematics, \\ Science Park 904, Postbus 94248, 1090 GE Amsterdam, The Netherlands \\ E-mail: mmguica@lpthe.jussieu.fr, k.skenderis@uva.nl, m.taylor@uva.nl, \\ b.c.vanrees@uva.nl
}

ABSTRACT: We discuss holography for Schrödinger solutions of both topologically massive gravity in three dimensions and massive vector theories in $(d+1)$ dimensions. In both cases the dual field theory can be viewed as a $d$-dimensional conformal field theory (two dimensional in the case of TMG) deformed by certain operators that respect the Schrödinger symmetry. These operators are irrelevant from the viewpoint of the relativistic conformal group but they are exactly marginal with respect to the non-relativistic conformal group. The spectrum of linear fluctuations around the background solutions corresponds to operators that are labeled by their scaling dimension and the lightcone momentum $k_{v}$. We set up the holographic dictionary and compute 2-point functions of these operators both holographically and in field theory using conformal perturbation theory and find agreement. The counterterms needed for holographic renormalization are non-local in the $v$ lightcone direction.

KEYwords: Gauge-gravity correspondence, AdS-CFT Correspondence, Holography and condensed matter physics (AdS/CMT)

ARXIV EPRINT: 1008.1991 


\section{Contents}

1 Introduction and summary 2

2 Gravity theories $\quad 8$

2.1 Topologically massive gravity $\quad 9$

$\begin{array}{ll}2.2 \text { Massive vector model } & 11\end{array}$

3 Asymptotics in the gauge/gravity duality 12

$\begin{array}{lll}3.1 & \text { Interpretation as a deformation } & 13\end{array}$

4 Field theory analysis $\quad \mathbf{1 4}$

4.1 Marginal Schrödinger invariant deformations 14

$\begin{array}{ll}4.2 & \text { Proof of marginality } \\ & 16\end{array}$

4.3 Deformations of two-dimensional CFTs: TMG example 18

4.4 Other deformations of 2d CFTs: general $z \quad 22$

$\begin{array}{ll}4.5 & \text { Massive vector model with } z=2\end{array}$

4.6 Higher spacetime dimensions 26

$\begin{array}{lll}4.7 & \text { Stress energy tensor } & 30\end{array}$

$5 \quad$ TMG and the null warped background $\quad 33$

5.1 The variational principle for TMG 33

5.2 The dilatation operator in the null warped background 36

6 Scalar field in the Schrödinger background 39

$\begin{array}{lll}6.1 & \text { Action and equations of motion } & 39\end{array}$

6.2 The dilatation operator and asymptotic expansions 40

$\begin{array}{lll}6.3 & \text { Renormalization of the action } & 42\end{array}$

6.4 Interpretation of the result 43

$\begin{array}{lll}7 & \text { Linearized analysis for TMG } & 44\end{array}$

7.1 Linearized bulk equations and solutions 44

$\begin{array}{lll}7.2 & \text { On-shell action } & 49\end{array}$

8 Linearized analysis for the massive vector model $\quad 52$

$\begin{array}{lll}8.1 & \text { Interpretation } & 57\end{array}$

9 Conclusions $\quad 57$ 


\section{Introduction and summary}

Gauge/gravity dualities have become an important new tool in extracting strong coupling physics. The best understood examples of such dualities involve relativistic quantum field theories. Strongly coupled non-relativistic QFTs are common place in condensed matter physics and as such there would be many interesting applications had one had under control holographic dualities involving non-relativistic QFTs. Motivated by such applications [1, 2] initiated a discussion of holography ${ }^{1}$ for $(d+1)$ dimensional spacetimes with metric,

$$
d s^{2}=-\frac{b^{2} d u^{2}}{r^{4}}+\frac{2 d u d v+d x^{i} d x^{i}+d r^{2}}{r^{2}},
$$

with $i \in\{1, \ldots, d-2\}$. The isometries of this metric form the so-called Schrödinger group, whose generators are given by:

$$
\begin{aligned}
& \mathcal{H}: u \rightarrow u+a, \\
& \mathcal{M}: v \rightarrow v+a \text {, } \\
& \mathcal{D}: r \rightarrow(1-a) r, \quad u \rightarrow(1-a)^{2} u, \quad v \rightarrow v, \quad x^{i} \rightarrow(1-a) x^{i} \\
& \mathcal{C}: r \rightarrow(1-a u) r, \quad u \rightarrow(1-a u) u, \quad v \rightarrow v+\frac{a}{2}\left(x^{i} x^{i}+r^{2}\right), \quad x^{i} \rightarrow(1-a u) x^{i}
\end{aligned}
$$

plus rotations, translations and Galilean boosts in the $x^{i}$ directions. Here $(\mathcal{D}, \mathcal{C})$ are the generators of non-relativistic scale transformations with dynamical exponent $z=2$ and special conformal symmetries, respectively.

It was initially suggested that the metric (1.1) could play the role of a background for the holographic study of critical non-relativistic systems in $(d-1)$ spacetime dimensions, for example fermions at unitarity, which have the same symmetry group. For such theories the specific realization of the Schrödinger group on (1.1) dictates that the $(d-1)$ dimensional theory should live on the spacetime spanned by the coordinates $\left(u, x^{i}\right)$ with $u$ playing the role of time. In this setup one considers operators $\mathcal{O}_{\Delta_{s}, m}\left(u, x^{i}\right)$ of definite scaling dimension $\Delta_{s}$ and of charge $m$ under the symmetry $\mathcal{M}$. This charge $m$, which corresponds to momentum in the $v$ direction, would then have to be identified with a discrete quantum number like particle number. In order to discretize the possible values of $m$ one therefore needs to compactify the $v$ direction. This procedure is however very nontrivial as in general quantum corrections become important and one cannot trust the metric (1.1) with a compact null direction [5]. Recent work aiming at obtaining solutions without such a compact direction can be found in [6].

The metric (1.1) has constant negative scalar curvature but it is not an asymptotically locally AdS (or AlAdS) spacetime. (We review this further below.) This property sets it apart from other gauge/gravity dualities, which are based upon the notion of AlAdS spacetimes. It should be stressed that this implies that a priori one cannot extend any of the standard holographic results to such spacetimes. For example, there are no guarantees that the dual description has the form of an ordinary local, renormalizable quantum field theory.

\footnotetext{
${ }^{1}$ Note that a Kaluza-Klein framework for the geometric realization of Schrödinger symmetries was introduced much earlier, in [3]; the relation of this work to the holographic framework is discussed in [4].
} 
The principal aim of this paper is to understand how holography works for these spacetimes. To avoid the complications of a compact null direction, we consider (1.1) with $v$ non-compact. The effects of such a compactification may be considered afterwards but this issue will be for the most part suppressed in this paper. We will present evidence that the spacetime (1.1) is dual to a $d$-dimensional quantum field theory that is non-local in the $v$ direction. More precisely, our viewpoint is that the dual quantum field theory can be obtained from a $d$-dimensional conformal field theory by deforming with operators that respect the Schrödinger symmetry. These operators are irrelevant from the perspective of the relativistic conformal group but they are exactly marginal from the perspective of the non-relativistic conformal group. As a first consistency check, notice that an irrelevant deformation generally changes the UV properties of the theory and this explains why the dual gravity solution is no longer asymptotically anti-de Sitter. In our context the UV properties are now governed by the Schrödinger group and indeed the solution realizes this symmetry geometrically.

Since the deformed QFT is Schrödinger symmetric, we should organize it in a way that makes this symmetry manifest. To discuss this let us first recall that any relativistic $d$-dimensional CFT is also invariant under the $(d-2)$ dimensional Schrödinger group. This follows from the fact the conformal group in $d$ dimensions has as a subgroup the $(d-2$ )-dimensional Schrödinger group (as first discussed in [7] for $d=4){ }^{2}$ It follows that it should be possible to rewrite the correlation function in a form that manifests the Schrödinger symmetry. Indeed, as discussed recently in [8], the mixed representation of the 2-point function where one Fourier transforms over the lightcone coordinate $v$ brings the relativistic 2-point function into the form dictated by the Schrödinger symmetry [9].

Schrödinger solutions have arisen as solutions of various gravitational theories; in this paper we will focus on topologically massive gravity (TMG) in three dimensions and the massive vector model used by Son [1]. Schrödinger solutions of TMG arise for a specific value of the coupling, namely $\mu=3$, and in earlier literature have been referred to as null warped $A d S_{3}$ backgrounds. The operator $X_{v v}$ which deforms the dual theory has relativistic scaling dimensions $(h, \bar{h})=(3,1)$, breaks the Lorentz invariance and is dual to a null component of the extrinsic curvature, as discussed in [10,11]. Gravity coupled to a cosmological constant and a massive vector with $m^{2}=2 d$ also admits Schrödinger solutions in general dimension $d$; moreover the solution for $d=4$ can be understood as a consistent truncation of a decoupling limit of TsT transformed branes [5]. In this case the deforming operator $X_{v}$ has relativistic scaling dimension $d+1$ and is dual to a null component of the massive vector.

As mentioned above, in theories with Schrödinger invariance operators are labeled by the non-relativistic scaling dimension $\Delta_{s}$ and the eigenvalue of $\mathcal{M}$, which in our case is the right-moving momentum $k_{v}$. We will therefore Fourier transform in the right moving sector

\footnotetext{
${ }^{2}$ The embedding is the following. Choosing lightcone coordinates $u, v$, the relativistic momentum generators $P_{u}$ and $P_{v}$ are identified with $\mathcal{H}$ and $\mathcal{M}$, respectively, the non-relativistic scaling generator $\mathcal{D}$ is a linear combination of the relativistic scaling generator and a boost in the $u v$ direction and $\mathcal{C}$ is related to a relativistic special conformal generator. Translations, rotations and Galilean boosts and related to translations and rotations in the relativistic theory. More details can be found, for example, in [1] or [5].
} 
and consider the operators with different $k_{v}$ as independent operators. If $v$ is compact then $k_{v}$ would be a discrete label, but as mentioned above we do not compactify $v$ and therefore $k_{v}$ is a continuous variable. The deformation of the original CFT for the case of the massive vector is of the form,

$$
S_{\mathrm{CFT}} \rightarrow S_{\mathrm{CFT}}+\int d^{d-2} x d u d v b^{\mu} X_{\mu}\left(v, u, x^{i}\right)=S_{\mathrm{CFT}}+\int d^{d-2} x d u b^{\mu} \tilde{X}_{\mu}\left(k_{v}=0, u, x^{i}\right),
$$

where $S_{\mathrm{CFT}}$ is the original CFT action, $b^{\mu}$ is a constant null vector whose only non-vanishing component is $b^{v}=b$ and $\tilde{X}_{\mu}$ is the Fourier transform of $X_{\mu}$ in the $v$ direction. In the rest of this paper we will often drop the tilde and (with abuse of notation) denote an operator and its Fourier transform with respect to $v$ by the same name. We see from (1.3) that the theory is deformed by an operator of zero lightcone momentum. The operator $X_{\mu}$ has dimension $d+1$ from the perspective of the relativistic CFT and breaks the Lorentz symmetry. Since its (relativistic) dimension is different from $d$, one can use relativistic scaling $^{3}$ to set $b=1$. This operator however is exactly marginal from the perspective of the Schrödinger symmetry, i.e. its non-relativistic scaling dimension is $\Delta_{s}=d$ and this implies that the action (1.3) has Schrödinger invariance. These facts have an exact counterpart in the bulk: one can set $b=1$ in (1.1) by either by a bulk diffeomorphism that scales all coordinates by $b$ (corresponding to the relativistic rescaling) or by rescaling the lightcone coordinates as in footnote 3 and the metric has Schrödinger isometries. The discussion for the case of TMG is similar: one replaces $b^{\mu}$ by the symmetric null tensor $b^{\mu \nu}$ with the only non-zero component being $b^{v v}=-b^{2}$ and $X_{\mu}$ by $X_{\mu \nu}$, where $X_{\mu \nu}$ has relativistic scaling $(3,1)$ and non-relativistic scaling $\Delta_{s}=2$.

To show that the deformation is exactly marginal we have to show the non-relativistic scaling dimension of deforming operator, which was equal to $\Delta_{s}=d$ in the original CFT, remains equal to $\Delta_{s}=d$ in the deformed theory. This amounts to showing that the 2-point function of this operator in the deformed theory is the same as the 2-point function in the original CFT and this can be proven using conformal perturbation theory. In more detail, consider for concreteness the case of the massive vectors, then one needs to establish that for any $n$

$$
\lim _{k_{v} \rightarrow 0}\left\langle X_{v}\left(k_{v}\right)\left(\prod_{i=1}^{n} \int d^{d-2} x_{i} d u_{i} b^{\mu} X_{\mu}\left(k_{v}=0\right)\right) X_{v}\left(-k_{v}\right)\right\rangle_{C F T}=0
$$

where the expectation value is taken in the original CFT and to avoid clutter we only display the $k_{v}$ dependence. One can show that

$$
\left\langle X_{v}\left(k_{v}\right)\left(\prod_{i=1}^{n} b^{\mu} X_{\mu}\left(k_{v}=0\right)\right) X_{v}\left(-k_{v}\right)\right\rangle_{\mathrm{CFT}}=\left\langle X_{v}\left(k_{v}\right) X_{v}\left(-k_{v}\right)\right\rangle_{\mathrm{CFT}}\left(b^{v} k_{v}\right)^{n} f\left(\log k_{v}, \ldots\right)
$$

\footnotetext{
${ }^{3}$ Alternatively, one can set $b=1$ by rescaling of the lightcone coordinates, $u \rightarrow u / b, v \rightarrow v b$. This transformation is a composition of a relativistic scaling with parameter $b$ and a Schrödinger scaling with parameter $1 / b$.
} 
where $f\left(\log k_{v}, \ldots\right)$ is a dimensionless function that carries the dependence on the positions of the operators and is at most logarithmically dependent on $k_{v}$. Taking the limit $k_{v} \rightarrow 0$ we indeed find that the rhs of (1.5) vanishes and thus (1.4) is satisfied. Consequently the operator $X_{v}\left(k_{v}=0\right)$ is exactly marginal.

The same computation shows that operators with $k_{v} \neq 0$ will in general acquire an anomalous dimension,

$$
\Delta_{s}=\Delta_{s}(b=0)+\sum_{n>0} c_{n}\left(b k_{v}\right)^{n}
$$

where $\Delta_{s}(b=0)$ is the non-relativistic scaling dimension in the original CFT and the $c_{n}$ are computable numerical coefficients. This discussion holds not only for $X_{v}\left(k_{v}\right)$ but also for general composite operators $\mathcal{O}\left(k_{v}\right)$, i.e. in general, when $k_{v} \neq 0$, they acquire anomalous dimensions in the deformed theory. We will discuss explicit examples in section 4 .

Note that $b^{\mu}$ appears always in the combination $b^{v} k_{v}$ and this quantity is invariant under the rescalings (discussed above) that can set $b$ to any non-zero value. Later on, when we study in section 4 specific examples using conformal perturbation theory to leading order the small parameter will be $b^{v} k_{v}$.

The fact that $\Delta_{s}$ depends on $k_{v}$ has several important consequences. Correlation functions of composite operators in general require renormalization and the corresponding counterterms contain poles when $\Delta_{s}$ takes integer values because new infinities arise when $\Delta_{s}$ is an integer. The dependence of $\Delta_{s}$ on $k_{v}$ then implies that the counterterms are non-polynomial in $k_{v}$ (when $b k_{v} \ll 1$, they are polynomial but contain an infinite number of $k_{v}$ factors) and thus non-local in the $v$ direction.

The stress energy tensor in the field theory is somewhat more subtle because there are actually two related stress energy operators. One is the operator that couples to the metric; this is a natural operator when considering the theory as a deformation of the CFT. This operator however is not conserved except at $b=0$. The second operator is the one that couples to the vielbein; this tensor is not symmetric but it is conserved and it is the natural operator to consider in the deformed theory. We will illustrate this point with a toy Schrödinger invariant field theory in section 4.

We now move to the gravitational side. In all other examples of holography one sets up a holographic dictionary as follows: one first derives the most general asymptotic solutions of the field equations consistent with the boundary conditions. Substituting these solutions into the on-shell action $S$ allows one to regulate the volume divergences and derive a covariant local boundary counterterm action $S_{\text {ct }}$ which renormalizes the action, via $S_{\text {ren }}=S+S_{\text {ct }}$ [12]. Renormalized one point functions in the presence of sources are then derived by applying the GKPW prescription $[13,14]$ to the renormalized action, namely:

$$
\langle\mathcal{O}\rangle=\frac{1}{\sqrt{g}} \frac{\delta S_{\text {ren }}}{\delta \Phi_{(0)}}
$$

where the boundary value $\Phi_{(0)}$ acts as a source for the operator $\mathcal{O}$. Higher correlation functions are obtained by further functional differentiation; to obtain $n$-point functions one will need exact regular solutions of the bulk field equations to order $(n-1)$ in fluctuations. 
The first question that we address is whether such a holographic dictionary can be set up for probe scalar operators, dual to minimally coupled scalar fields. We find that indeed there is such a dictionary, but with a key conceptual difference to all earlier examples of holography: the boundary counterterms are non-local in the lightcone direction $v$. This is consistent with the the field theory discussion which implies that, once we turn on a source for an operator with non-zero $k_{v}$, there would generically be counterterms that depend on $\left(b k_{v}\right)^{n}$ for all $n$, and thus provides structural evidence for the holographic duality. As previously noted in $[1,2,15]$ the Schrödinger dimension for a scalar operator has the closed form:

$$
\Delta_{s}=\frac{d}{2}+\sqrt{\left(\frac{d}{2}\right)^{2}+m^{2}+b^{2} k_{v}^{2}},
$$

where $m^{2}$ is the mass squared of the bulk scalar field (in units of the curvature radius). This indicates that the series expansion like the one in (1.6) should resum the square root form (1.8).

We then focus on the gravitational sector of TMG and the massive vector theories. As a first step we consider linearized fluctuations about the Schrödinger backgrounds. We consider here the linearized equations in radial axial gauge $\left(h_{r i}=h_{r r}=0\right)$ such that

$$
d s^{2}=-\frac{b^{2} d u^{2}}{r^{4}}+\frac{2 d u d v+d r^{2}}{r^{2}}+\frac{h_{i j}}{r^{2}} d x^{i} d x^{j}
$$

along with vector fluctuations in the vector model. We give the general solutions for these linearized fluctuations in sections 7 and 8. In particular, we note that the number of independent solutions corresponds to the correct number of sources for dual operators and their expectation values (subject to constraints related to dual operator Ward identities).

These solutions have a number of interesting features which we now briefly summarize. Both models admit two distinct sets of solutions to the linearized equations, which we distinguish with superscripts ' $T$ ' and ' $X$ '. The ' $T$ ' solutions are associated with the dual stress energy tensor, whilst the ' $\mathrm{X}$ ' solutions are associated with the dual deforming operator. Note however that all bulk field fluctuations are non-zero in both sets of solutions.

Looking first at the ' $\mathrm{X}$ ' solutions, we see that indeed these exhibit the behavior expected for a bulk field dual to an operator with a $k_{v}$ dependent scaling dimension. In the case of TMG, the leading component of the fluctuation of the extrinsic curvature, $\bar{\kappa}_{u u}$, acts as a source for the dual operator $X_{v v}$. The asymptotic expansion of this linearized fluctuation is

$$
\begin{aligned}
\bar{\kappa}_{u u} & =\bar{\kappa}_{(0) u u} r^{-\Delta_{s}-2}(1+\cdots)+\bar{\kappa}_{\left(2 \Delta_{s}-2\right) u u} r^{\Delta_{s}-4}(1+\cdots), \\
\Delta_{s} & =1+\sqrt{1+b^{2} k_{v}^{2}}
\end{aligned}
$$

where the ellipses denote subleading terms as $r \rightarrow 0$. Then $\bar{\kappa}_{(0) u u}$ acts as a source for the dual operator $X_{v v}$, and $\Delta_{s}$ is its scaling dimension; moreover the two point function of this operator is indeed found to be of the expected Schrödinger form. The scaling dimension depends on the lightcone momentum, as shown in the field theory, and resums into the square root. At $b=0$ the Schrödinger dimension is in agreement with that of a relativistic dimension $(3,1)$ operator, as required by the AdS/CFT dictionary. 
The case of the massive vector is analogous: the ' $\mathrm{X}$ ' solutions are associated with the dual operators $X_{u}$ and $X_{v}$, the latter of which is the deforming operator. The asymptotic expansions indicate that the Schrödinger dimensions of these operators are respectively:

$$
\Delta_{s}\left(X_{v}\right)=1+\sqrt{1+b^{2} k_{v}^{2}} ; \quad \Delta_{s}\left(X_{u}\right)=1+\sqrt{9+b^{2} k_{v}^{2}},
$$

which are consistent with the CFT operator dimensions at $b=0$ and are again expressed as closed forms in $\left(b k_{v}\right)$.

An important feature of both these solutions is that they diverge faster as $r \rightarrow 0$ than the background solution. This is because the operators with non-zero $k_{v}$ renormalize and while these operators were marginal (w.r.t. Schrödinger scaling) at $b=0$ they become irrelevant when $b \neq 0$. Irrelevant operators change the UV behavior of the theory and should hence modify the leading asymptotics of the holographic dual. This is precisely what happens here: the faster rate near $r \rightarrow 0$ is precisely that dictated by the anomalous dimension.

Now let us turn to the ' $\mathrm{T}$ ' modes. In TMG the metric perturbation takes the form:

$$
\begin{aligned}
h_{u u}^{T} & =\frac{1}{r^{2}} h_{(-2) u u}+\tilde{h}_{(0) u u} \log \left(r^{2}\right)+h_{(0) u u}+r^{2} h_{(2) u u} \\
h_{u v}^{T} & =\frac{1}{r^{2}} h_{(-2) u v}+\tilde{h}_{(0) u v} \log \left(r^{2}\right)+h_{(0) u v}+r^{2} h_{(2) u v} \\
h_{v v}^{T} & =h_{(0) v v}+r^{2} h_{(2) v v},
\end{aligned}
$$

with analogous results for the massive vector model. The precise form of the coefficients $h_{(a) i j}$ as functions of $(u, v)$ is given in section 7 ; there are six independent coefficients subject to three constraints. The metric perturbation diverges faster as $r \rightarrow 0$ than the background solution; it does not respect the falloff conditions discussed in [16]. This is associated with the fact that certain components of the dual stress tensor are irrelevant with respect to the Schrödinger dilatation symmetry. It is possible to impose by hand the constraint that the metric perturbations should not blow up faster than the background metric as $r \rightarrow 0$, but this generically imposes constraints on the operator sources in the dual field theory. In previous works such as $[1,17,18]$ only such solutions with constrained asymptotics were discussed, and indeed the fact that the sources were apparently constrained was already noticed in [17].

These ' $\mathrm{T}$ ' modes should correspond to the stress energy tensor in the dual field theory. However, as noted above, there are subtleties in setting up the holographic dictionary in this case. One issue is the fact that one component of the stress energy tensor is an irrelevant operator, as mentioned above. The other is the fact that the conserved stress energy tensor for the field theory should couple to the vielbein, rather than the metric, and therefore, as noted in [18], the appropriate variational problem in the bulk corresponds to specifying boundary conditions for the vielbein. This involves reformulating the bulk theories in a vielbein formulation and will be discussed elsewhere.

The fact that the Schrödinger spacetime is obtained from anti-de Sitter by irrelevant deformations was already briefly noted in [1]. In [5] the relation of Schrödinger to anti-de 
Sitter via TsT transformations was used to argue that the dual field theory should be a null dipole theory; see also $[17,19]$ for additional discussion of the TsT transformations required. This observation is consistent with the view espoused here: the irrelevant deformations should resum into a null dipole theory which respects Schrödinger invariance. Note that all terms in the dipole theory are exactly marginal w.r.t. the Schrödinger symmetry. We postpone to subsequent work full exploration of the null dipole structure, since null dipole theories have not been developed in earlier literature and their properties differ qualitatively from the spacelike dipole theories developed in [20-22].

Whilst our viewpoint is consistent with the earlier proposals of [1] and [5] in the massive vector case, a somewhat different proposal has been made for the holographic dual to null warped backgrounds of TMG. In [23] it was suggested that the holographic dual to the null warped background should be a two-dimensional CFT with certain central charges $\left(c_{L}, c_{R}\right)$. Applying the Cardy formula at finite temperature using these central charges gives an entropy in agreement with that of a black hole which has null warped asymptotics. This agreement is certainly thought-provoking, but there are conceptual challenges with the suggestion that the dual field theory is a conformal field theory. When $b^{2}$ is small we can treat the solution as a linear perturbation around AdS and the spacetime (1.1) can be interpreted using the standard AdS/CFT dictionary at the linearized level. Using the TMG holographic dictionary derived in [10], the $b^{2}$ term acts as a source for the dimension $(3,1)$ irrelevant operator in the dual CFT. Therefore, the dual interpretation of null warped solutions of TMG at finite $b^{2}$ should indeed be in terms of a CFT deformed by irrelevant operators, which are however exactly marginal from the perspective of the Schrödinger symmetry.

The outline of the paper is as follows. In the next section we review two gravitational models which admit Schrödinger solutions, namely topologically massive gravity in three dimensions and the massive vector model. In section 3 we discuss the modified asymptotics of the Schrödinger metrics and how these show that the dual theory is obtained from specific irrelevant deformations of a relativistic conformal field theory. We show in section 4 that these deformations are exactly marginal with respect to the Schrödinger group and use conformal perturbation theory to discuss how the dimensions of operators change in the deformed theory. In section 5 we begin the holographic analysis by setting up the correct variational principle for TMG and deriving the dilatation operator at linearized level. In section 6 we discuss the bulk computation of the two-point function of a scalar operator. We consider the linearized analysis for TMG in section 7, and present the general solutions of the linearized equations. We set up a holographic dictionary for the deforming operator, and show that its two point function is of the expected form. We treat the linearized problem in the massive vector model in $d=2$ and give the general solution of the linearized equations of motion in section 8. We discuss our conclusions and open questions in section 9.

\section{Gravity theories}

Schrödinger backgrounds arise as solutions to a variety of gravitational theories. In this paper we will consider topologically massive gravity and massive vector models and here 
we briefly review both theories. Throughout this paper we use the following conventions for the curvatures:

$$
R_{\mu \nu \rho}^{\sigma}=\partial_{\nu} \Gamma_{\mu \rho}^{\sigma}+\Gamma_{\mu \rho}^{\lambda} \Gamma_{\nu \lambda}^{\sigma}-(\mu \leftrightarrow \nu), \quad R_{\mu \rho}=R_{\mu \sigma \rho}^{\sigma}
$$

so that for the metric $G_{\mu \nu}$ given by (1.1) one obtains:

$$
R_{\mu \nu}=-d G_{\mu \nu}+(d+2) \frac{b^{2}}{r^{4}} \delta_{\mu}^{u} \delta_{\nu}^{u}
$$

which can be used to verify the formulae below.

\subsection{Topologically massive gravity}

Topologically massive gravity (TMG) is a three-dimensional theory of gravity where the usual Einstein-Hilbert action is supplemented with a gravitational Chern-Simons term. The total action reads:

$$
S=\frac{1}{16 \pi G_{N}} \int d^{3} x \sqrt{-G}\left(R-2 \Lambda+\frac{1}{2 \mu} \epsilon^{\lambda \mu \nu}\left(\Gamma_{\lambda \sigma}^{\rho} \partial_{\mu} \Gamma_{\rho \nu}^{\sigma}+\frac{2}{3} \Gamma_{\lambda \sigma}^{\rho} \Gamma_{\mu \tau}^{\sigma} \Gamma_{\nu \rho}^{\tau}\right)\right)
$$

where $\Gamma_{\mu \nu}^{\lambda}$ are the connection coefficients associated to the metric $G_{\mu \nu}$ and where we use the covariant $\epsilon$-symbol such that $\sqrt{-G} \epsilon^{u v r}=1$, with $r$ the radial direction in (1.1). Variation of the action results in the equations of motion:

$$
R_{\mu \nu}-\frac{1}{2} G_{\mu \nu} R+\Lambda G_{\mu \nu}+\frac{1}{2 \mu}\left(\epsilon_{\mu}{ }^{\rho \sigma} \nabla_{\rho} R_{\sigma \nu}+\epsilon_{\nu}{ }^{\rho \sigma} \nabla_{\rho} R_{\sigma \mu}\right)=0 .
$$

We henceforth set $\Lambda=-1$. Taking the trace of this equation results in $R=-6$, so all solutions to TMG have a constant negative Ricci scalar. Furthermore, any Einstein metric in three dimensions has $R_{\mu \nu}=-2 G_{\mu \nu}$ and is easily seen to be a solution of (2.4) as well. In particular, $\mathrm{AdS}_{3}$ is a solution of TMG for all values of $\mu$. For generic values of $\mu$ there also exist so-called warped $A d S_{3}$ spaces, see [23] for their properties. In the specific case $\mu=3$ we find null warped $A d S_{s}$ as a solution,

$$
d s^{2}=-\frac{b^{2} d u^{2}}{r^{4}}+\frac{2 d u d v+d r^{2}}{r^{2}}
$$

which is precisely (1.1) with $d=2$. Therefore null warped $A d S_{3}$ is equivalent to the threedimensional Schrödinger spacetime. In solving the TMG field equations $b^{2}$ is an arbitrary real parameter, so, in particular, $b^{2}$ can have either sign. In the massive vector model we discuss below this is not the case and $b^{2}$ is necessarily positive. Furthermore, the analysis in section 6 seems to indicate that $b^{2}>0$ is necessary for stability. For these reasons we will continue to use the notation $b^{2}$ even when we consider the metric as a solution to TMG.

In [10] details of the holographic dictionary for TMG were presented. The most important feature for our purposes is that, since the equations of motion of TMG are third order in derivatives, we need to specify not only the boundary metric but also (a component of) the extrinsic curvature in order to find a unique bulk solution. When we apply 
gauge/gravity duality to TMG with a negative cosmological constant, the extra boundary data corresponds to the source of an extra operator. Therefore, besides the boundary energy-momentum tensor $T_{i j}$, which couples to the boundary metric $g_{(0) i j}$, we also have a new operator $X_{v v}$ which couples to the leading coefficient of the radial expansion of the (uu) component of the extrinsic curvature. It was shown in [10] that the operator $X_{v v}$ has weights $\left(h_{L}, h_{R}\right)=\frac{1}{2}(\mu+3, \mu-1)$. (Strictly speaking, the analysis of [10] was for $0<\mu<2$, but the extension to general $\mu$ is straightforward.) Moreover, a precise relation between the extra boundary data and the presence of a new operator in the dual field theory was established and the two-point functions of these operators around the state dual to an AdS background were computed.

Working to leading order in $b^{2}$, we can interpret the Schrödinger solution (2.5) using the linearized AdS/CFT dictionary. Noting that the metric

$$
d s^{2}=\frac{h_{i j} d x^{i} d x^{j}+2 d u d v+d r^{2}}{r^{2}}
$$

describes linearized perturbations about an AdS background, then the results of [10] indicate that the general solution to the linearized equations of motion at $\mu=3$ in which $h_{i j}$ depends only on the radial coordinate is:

$$
\begin{aligned}
h_{u v} & =h_{(0) u v} \\
h_{u u} & =\frac{1}{r^{2}} h_{(-2) u u}+h_{(0) u u}+r^{2} h_{(2) u u} \\
h_{v v} & =h_{(0) v v}+r^{2} h_{(2) v v}+r^{4} h_{(4) v v}
\end{aligned}
$$

which is expressed in terms of seven independent integration constants. If only the constant $h_{(-2) u u} \equiv-b^{2}$ is non-zero, the linearized solution is precisely the Schrödinger solution, which of course also solves the full non-linear equations of motion. However, applying the holographic dictionary derived at the linearized level, $-b^{2}$ acts as a (constant) source for the dimension $(3,1)$ operator $X_{v v}$. Therefore, the dual field theory, at least to leading order in $b^{2}$, must be a null irrelevant deformation of the original conformal field theory.

Applying the holographic one point functions applicable at the linearized level which are given in [10] yields:

$$
\left\langle T_{u u}\right\rangle=\left\langle T_{v v}\right\rangle=\left\langle X_{v v}\right\rangle=0
$$

for this background. This indicates that the background corresponds to the vacuum of the deformed theory. It is also interesting to note that a linearized solution with constant $h_{(-2) u u}=h_{(2) u u}$ also solves the non-linear equations of motion. The resulting background:

$$
d s^{2}=\frac{d r^{2}}{r^{2}}+\frac{1}{r^{2}}\left(-b^{2}\left(\frac{1}{r^{2}}+r^{2}\right) d u^{2}+2 d u d v\right)
$$

is Schrödinger in the global coordinates introduced by [24]. Applying the linearized holographic one point functions given in [10] to this background, we note that there is still a constant source for the dimension $(3,1)$ operator $X_{v v}$ but:

$$
\left\langle T_{u u}\right\rangle=-\frac{1}{6 G_{N}} b^{2},
$$


with $\left\langle T_{v v}\right\rangle=\left\langle X_{v v}\right\rangle=0$. This suggests that the background should correspond to the deformed field theory in a different state, with $\left\langle T_{u u}\right\rangle$ presumably related to the Casimir energy. We should emphasize however that these formulae for the holographic one point functions apply only at leading order in $b^{2}$. Understanding the dictionary at finite $b^{2}$ is far more subtle; it requires us to go beyond asymptotically locally AdS spacetimes and is the focus of section 7 .

Note that the holographic dictionary for TMG reflects the various problems of the theory. The theory contains negative norm states and it is thus unlikely that TMG in itself could be a consistent theory of quantum gravity. Nevertheless it remains a rich and interesting toy model offering gravitational dynamics in three bulk dimensions, logarithmic correlation functions (for $\mu=1$ ) and, as we exploit here, it allows Schrödinger backgrounds as solutions.

\subsection{Massive vector model}

The massive vector model consists of Einstein gravity coupled to a massive vector field. The action is

$$
S=\frac{1}{16 \pi G_{N}} \int d^{d+1} x \sqrt{-G}\left(R-2 \Lambda-\frac{1}{4} F_{\mu \nu} F^{\mu \nu}-\frac{1}{2} m^{2} A_{\mu} A^{\mu}\right)
$$

The equations of motion take the form

$$
\begin{aligned}
R_{\mu \nu}-\frac{1}{2} R G_{\mu \nu}+\Lambda G_{\mu \nu} & =\frac{1}{2} F_{\mu \rho} F_{\nu}{ }^{\rho}+\frac{1}{2} m^{2} A_{\mu} A_{\nu}-G_{\mu \nu}\left(\frac{1}{8} F_{\rho \sigma} F^{\rho \sigma}+\frac{1}{4} m^{2} A_{\rho} A^{\rho}\right) \\
\nabla^{\nu} F_{\nu \mu}-m^{2} A_{\mu} & =0
\end{aligned}
$$

The vector equations of motion also imply the identity $\nabla^{\mu} A_{\mu}=0$.

For $\Lambda=-d(d-1) / 2$ and $m^{2}=2 d$ we find the metric (1.1) and

$$
A=\frac{b}{r^{2}} d u \text {. }
$$

as a solution. Note that the dimension of the dual vector operator $X_{i}$ is $(d+1)$ and the linearized AdS/CFT dictionary implies that $b$ acts as a source for the operator $X_{v}$.

The solution with $d=4$ requires a massive bulk vector field with $m^{2}=8$. Such a vector field arises as one of the Kaluza-Klein modes in type IIB compactifications of the form $\mathrm{AdS}_{5} \times Y$ with $Y$ a Sasaki-Einstein manifold. A consistent truncation including this mode was found in [5], and this can be used to uplift the solution to a ten-dimensional solution of type IIB supergravity. The five-dimensional consistent truncation contains the metric, the massive vector field with $m^{2}=8$ and three scalars (one of which is massless) with a nontrivial potential. Further discussions of embeddings of Schrödinger solutions into string theory may be found in [25-31].

The solution presented above in the case of $d=4$ is related by a so-called TsT transformation to $\mathrm{AdS}_{5} \times Y[5,17]$. Following the chain of transformations, this implies that the field theory dual to a Schrödinger spacetime should be a null dipole theory. Dipole theories were originally introduced in [20] and arise from taking decoupling limit of branes 
in a background with a $B$ field with one leg longitudinal to the brane and one leg transverse to the brane. The structure of dipole theories is analogous to the better known non-commutative theories that arise on decoupling branes in a background with constant $B$ longitudinal to the brane worldvolume [32]. In general, to obtain a dipole theory, one starts with a local and Lorentz invariant field theory in $d$ dimensions. Then to every field $\Phi$ is assigned a vector $L_{\mu}$ where $\mu=1, \cdots d$; this is the dipole vector of the field. The fields can be scalars, fermions or have higher spin. Next, one defines a dipole product which is non-commutative:

$$
\Phi_{1} * \Phi_{2} \equiv \Phi_{1}\left(x-\frac{1}{2} L_{2}\right) \Phi_{2}\left(x+\frac{1}{2} L_{1}\right) .
$$

This defines an associative product provided that the vector assignment is additive, that is, $\Phi_{1} * \Phi_{2}$ is assigned the dipole vector $L_{1}+L_{2}$. For CPT symmetry, one requires that if $\Phi$ has dipole vector $L$ then the charge conjugate field, $\Phi^{\dagger}$, is assigned the dipole vector $-L$. Also gauge fields have zero dipole length. In most of the earlier literature, the case where the dipole vector $L$ is spacelike was considered. In the current context, however, the dipole vector is null, as we review below. In the case where $Y=S^{5}$, the TsT transformations change the dual field theory from $\mathcal{N}=4$ super Yang-Mills to a null dipole version of this theory. The analytic structure of quantities computed holographically should therefore match those expected for a null dipole theory. However, there is very little literature on null dipole theories. The analytic structure of spacelike dipole theories is believed to be closely related to that of non-commutative theories, which in turn is extremely subtle due to UV/IR mixing [33]. The analytic structure of null dipole theories should however be more similar to theories with light-like non-commutativity, which were argued in [34] to be unitary, and this is consistent with what we find here.

\section{Asymptotics in the gauge/gravity duality}

As already mentioned in the introduction, the Schrödinger spacetime is not an asymptotically locally AdS spacetime. As is reviewed in [12], any asymptotically locally AdS spacetime admits in the neighborhood of the conformal boundary $r \rightarrow 0$ a metric of the form:

$$
d s^{2}=\frac{d r^{2}}{r^{2}}+\frac{1}{r^{2}} g_{i j} d x^{i} d x^{j} \quad g_{i j}\left(r, x^{k}\right)=g_{(0) i j}\left(x^{k}\right)+\ldots
$$

where the dots represent terms that vanish as $r \rightarrow 0$ and $g_{(0) i j}$ is an arbitrary nondegenerate metric.

The relevance of this structure to holography is the following. Suppose we would like to use holography to compute certain field theoretic quantities. Just as in field theory computations, the holographic computation of these correlation functions suffers from divergences which need to be regularized and renormalized [12]. In this procedure of holographic renormalization the asymptotics of the spacetime play a crucial role: they are, via the equations of motion, directly related to the form of the divergences which need to be subtracted [35, 36]. For example, for asymptotically AdS spacetimes the supergravity equations of motion, combined with the asymptotics (3.1), guarantee that all divergences 
are local in the boundary data $[37,38]$. These divergences can therefore be subtracted by local counterterms as well. This is in agreement with the fact that the dual field theory is local and renormalizable, and the fact that the divergences on the gravity side have this form is strong structural evidence for gauge/gravity dualities.

For the case at hand, we find ourselves outside of the usual framework, since for nonzero $b$ the spacetime is no longer AlAdS. The standard results of holographic renormalization therefore do not directly carry over to this setting and in order to obtain correlation functions one has to build a holographic dictionary from first principles. Just as in field theory, it is imperative to understand systematically the structure of these divergences in order to discuss renormalization and obtain correct finite correlation functions. Notice that there is a priori no guarantee that the divergences will be local in the boundary data and in fact we will encounter certain nonlocality in the divergences below.

\subsection{Interpretation as a deformation}

In this paper we will explore the analytic structure on both sides of the duality. As a first step in this exploration it is interesting to take the limit $b \rightarrow 0$, for which the metric (1.1) reduces to that of empty AdS, and expand correlation functions perturbatively in the small parameter $b$. When $b$ is zero we simply recover the AdS metric in Poincaré coordinates, and results to leading order around $b=0$ may be obtained via the usual AdS/CFT dictionary. In particular, for small perturbations around the conformal vacuum we find the following radial expansion for the massive vector field $A_{\mu}$ :

$$
A_{i}=\frac{1}{r^{2}}\left(A_{(0) i}+\ldots+r^{d+2} A_{(d+2) i}+\ldots\right) .
$$

Holographically, $A_{(0) i}$ is interpreted as the source for a dual operator $X^{i}$ and its expectation value is related to the normalizable mode $A_{(d+2) i}$. Given the mass of the bulk vector field, the corresponding scaling dimension $\Delta$ of the dual operator $X^{i}$ is given by $(d+1)$.

By comparing the explicit gauge field solution (2.13) with (3.2) we find that, to first order, switching on $b$ can be interpreted holographically as an irrelevant deformation of the original CFT of the form:

$$
\int d^{D+2} x b^{v} X_{v}
$$

where $b^{v}=b$. Similarly, as we discussed earlier, for TMG the corresponding deformation is:

$$
\int d^{2} x b^{v v} X_{v v}
$$

with $b^{v v}=-b^{2}$.

These results are based on an analysis for small $b$. Given that the deforming operators are irrelevant (so the deformed theory appears non-renormalizable and thus uncontrollable in the UV) it would seem hard to extend these results to finite $b$. We have seen however that the linearized solution automatically solves the non-linear equations of the motion. This is related to the fact that the linearized solution has a new scaling symmetry, the dilatation of the Schröndinger group, which controls the UV behavior of the theory and 
the value of $b$ can be set to any value (provided it is non-zero) by an appropriate scaling of the lightcone coordinates (see footnote 3 ) while maintaining the new scaling symmetry. Thus the solution derived for small $b$ is automatically also a solution for large $b$. The counterpart of this statement on the QFT side is that the deforming operator is exactly marginal and after the deformation the theory finds itself at a non-relativistic fixed point.

One of the main questions is then to understand how the deformation changes the spectrum of operators. We will analyze this question on the field theory side using conformal perturbation theory. On the gravitational side, the same question amounts to solving the linearized equations of the motion.

\section{Field theory analysis}

From the previous discussion, Schrödinger backgrounds are dual to conformal field theories deformed by operators that respect the Schrödinger symmetry. In this section we will use conformal perturbation theory to show that the deforming operator is exactly marginal and then study how the spectrum of scaling dimensions changes in the deformed theory. We will only use general results that follow from conformal invariance, so our results are valid for any relativistic CFT, weakly or strongly coupled, that has in its spectrum operators of the right type. Similar arguments can be made for scale invariant theories with other dynamical exponents, $z \neq 2$, and these are discussed briefly in section 4.4 .

\subsection{Marginal Schrödinger invariant deformations}

Any relativistic $d$-dimensional conformal field theory is also invariant under the $(d-2)$ dimensional Schrödinger group. In particular the non-relativistic scaling dimension $\Delta_{s}$ is related to the relativistic scaling dimension $\Delta$ via $[1,5,7]$ :

$$
\Delta_{s}=\Delta+M_{v u}
$$

where $M_{v u}$ is the eigenvalue of the boost operator in the lightcone directions normalized such that $v$ and $u$ have eigenvalues +1 and -1 , respectively. This implies that one can break the relativistic conformal group while preserving the $(d-2)$-dimensional Schrödinger subgroup. Such deformations will necessarily also break the Lorentz invariance since the deforming operator must have a non-zero eigenvalue $M_{v u}$.

Consider now a conformal field theory deformed by an operator with Schrödinger scaling dimension $\Delta_{s}$. Such a deformation is irrelevant with respect to the Schrödinger symmetry when $\Delta_{s}>d$, marginal when $\Delta_{s}=d$ and relevant when $\Delta_{s}<d$. Consider first the case where we deform with a scalar operator. Then the deformation respects not only Schrödinger symmetry, but also the relativistic conformal symmetry, as it does not break rotational invariance in the $(u, v)$ directions; such marginal deformations of CFTs have been extensively explored. Suppose now that the operator is not a scalar, but rather a vector $X_{i}$ or a tensor $X_{i j}$, of Schrödinger dimension $d$. Such deformations can respect Schrödinger symmetry, but break the relativistic symmetry, provided that the sources are constant null vectors or tensors respectively, with components only along the $v$ directions. 
An example of such a deformation is:

$$
S_{\mathrm{CFT}} \rightarrow S_{\mathrm{CFT}}+\int d^{d-2} x d u d v b^{i} X_{i}\left(v, u, x^{i}\right)=S_{\mathrm{CFT}}+\int d^{d-2} x d u b^{i} \tilde{X}_{i}\left(k_{v}=0, u, x^{i}\right),
$$

with $b^{i}$ a constant null vector with non-vanishing component $b^{v} \equiv b$ and $\tilde{X}$ the Fourier transform of $X$ in the $v$ direction. This example is realized in the duality with the massive vector theory. In the case of TMG, the deformation of interest is by a tensor operator, namely:

$$
S_{\mathrm{CFT}} \rightarrow S_{\mathrm{CFT}}+\int d u d v b^{i j} X_{i j}\left(v, u, x^{i}\right)=S_{\mathrm{CFT}}+\int d u b^{i j} \tilde{X}_{i j}\left(k_{v}=0, u, x^{i}\right),
$$

where the only non-vanishing component of $b^{i j}$ is $b^{v v}=-b^{2}$. In both cases the deformations are marginal from the perspective of the Schrödinger symmetry, i.e. whilst both operators are irrelevant from the perspective of the relativistic conformal symmetry, they have nonrelativistic scaling dimensions $\Delta_{s}=d$. The deformations break the Lorentz symmetry, but respect all rotations, translations and boosts of the Schrödinger group. In the specific case of two dimensions, the deformations in addition respect the infinite-dimensional algebra associated with analytic coordinate transformations $u \rightarrow u^{\prime}=u^{\prime}(u)$.

To illustrate the general idea let us give a simple explicit example: consider the twodimensional action

$$
S=\int d u d v\left(\partial_{u} \Phi \partial_{v} \Phi+b \partial_{u} \Phi\left(\partial_{v} \Phi\right)^{3}\right)
$$

The first term preserves full two-dimensional relativistic conformal invariance whilst the second corresponds to a deformation of the relativistic conformal field theory by a dimension four operator, with scaling weights $(3,1)$. The deformation manifestly breaks both relativistic conformal invariance and two-dimensional Lorentz invariance but preserves the Schrödinger invariance (at least at the classical level). More generally, one can observe that any deformation of the type:

$$
\delta S=\int d u d v\left(\partial_{u} \Phi \partial_{v} \Phi\right) f\left(\partial_{v} \Phi, \partial_{v}^{2} \Phi, \cdots\right)
$$

respects the Schrödinger symmetry at the classical level for any functional $f\left(\partial_{v} \Phi, \partial_{v}^{2} \Phi, \cdots\right)$.

Such deformations are manifestly marginal with respect to the Schrödinger symmetry, but one also needs to show that the deformations are exactly marginal. This requires proving that the non-relativistic dimensions of $X\left(k_{v}=0, u, x^{i}\right)$ remain equal to $\Delta_{s}=d$ in the deformed theory, which amounts to showing that the 2-point function of $X\left(k_{v}=0, u, x^{i}\right)$ in the deformed theory is the same as in the original theory. We can demonstrate this property using conformal perturbation theory as follows. Let us consider first the case of deformation by a vector operator with a constant null source. In conformal perturbation theory, the correction to the two point function in the deformed theory is expressed in terms of higher point functions in the conformal theory as:

$$
\begin{aligned}
\delta\left\langle\tilde{X}_{v}\left(k_{v}, k_{u}, k_{i}\right) \tilde{X}_{v}\left(-k_{v},-k_{u},-k_{i}\right)\right\rangle & \\
& =\sum_{n \geq 1} \frac{1}{n !}\left\langle\tilde{X}_{v}\left(k_{v}, k_{u}, k_{i}\right)\left(b \tilde{X}_{v}(0)\right)^{n} \tilde{X}_{v}\left(-k_{v},-k_{u},-k_{i}\right)\right\rangle_{\mathrm{CFT}} .
\end{aligned}
$$


Note that the operator insertions are at zero momentum. In the original CFT, the operator $\tilde{X}_{v}(0)$ has relativistic conformal dimension $(d+1)$ and Schrödinger dimension $d$, and transforms as a vector. This implies that the deformed theory is invariant under relativistic conformal symmetry provided that $b$ is transformed contravariantly, with relativistic scaling dimension minus one. Each additional insertion of $\left(b \tilde{X}_{v}(0)\right)$ therefore adds another factor of $b k_{v}$, so that:

$$
\begin{aligned}
\left\langle\tilde{X}_{v}\left(k_{v}, k_{u}, k_{i}\right)\right. & \left.\prod_{n}\left(b \tilde{X}_{v}(0)\right) \tilde{X}_{v}\left(-k_{v},-k_{u},-k_{i}\right)\right\rangle_{\mathrm{CFT}}= \\
& \left(b k_{v}\right)^{n}\left\langle\tilde{X}_{v}\left(k_{v}, k_{u}, k_{i}\right) \tilde{X}_{v}\left(-k_{v},-k_{u},-k_{i}\right)\right\rangle_{\mathrm{CFT}} f\left(\ln \left(k^{2} / \mu^{2}\right)\right) .
\end{aligned}
$$

As we will show below, relativistic conformal invariance implies that the scalar function $f$ can depend only logarithmically on the momentum. Since the CFT two point function is necessarily finite or vanishing at zero momentum, the operator at zero $k_{v}$ momentum receives no corrections to its two point function since:

$$
\left\langle\tilde{X}_{v}\left(0, k_{u}, k_{i}\right) \prod^{n}\left(b \tilde{X}_{v}(0)\right) \tilde{X}_{v}\left(0,-k_{u},-k_{i}\right)\right\rangle_{\mathrm{CFT}} \rightarrow 0
$$

for all $n \geq 1$. A similar argument implies that the operator at zero $k_{v}$ momentum remains orthogonal to all other operators. Thus the deforming operator is exactly marginal. However, the operators at non-zero $k_{v}$ momenta do receive corrections to their two point functions and thus to their scaling dimensions under Schrödinger symmetry; these corrections will be explicitly computed below.

\subsection{Proof of marginality}

The key identity required to prove that the operator $X\left(k_{v}=0\right)$ is exactly marginal is:

$$
\begin{array}{r}
\left\langle X_{v}\left(k_{v}\right)\left(\prod_{i=1}^{n} b^{\mu} \cdot X_{\mu}\left(k_{v}=0\right)\right) X_{v}\left(-k_{v}\right)\right\rangle_{\mathrm{CFT}} \\
\quad\left\langle X_{v}\left(k_{v}\right) X_{v}\left(-k_{v}\right)\right\rangle_{\mathrm{CFT}}\left(b^{v} k_{v}\right)^{n} f\left(\log k_{v}, \ldots\right)
\end{array}
$$

where $f\left(\log k_{v}, \ldots\right)$ is a dimensionless function that carries the dependence on the positions of the operators and is at most logarithmically dependent on $k_{v}$. The corresponding identity for TMG involves the operator $X_{v v}$ in the two dimensional conformal field theory.

In this section we will prove this identity in the case of two-dimensional CFTs. In two dimensions, both the massive vector and TMG cases involve deriving the structure of $(n+2)$-point functions of $(q, 1)$ operators, $X_{v \cdots v}$ ( $q v$-indices $)$, with themselves, in which $n$ of the operators are at zero momentum. We will do the computation in position space and in Euclidean signature and then Fourier transform afterwards. We thus need to compute

$$
\int\left(\prod_{i=2}^{n+1} d^{2} w_{i}\right)\left\langle X_{v \cdots v}\left(w_{1}, \bar{w}_{1}\right)\left(\prod_{i=2}^{n-1} X_{v \cdots v}\left(w_{i}, \bar{w}_{i}\right)\right) X_{v \cdots v}\left(w_{n+2}, \bar{w}_{n+2}\right)\right\rangle_{\mathrm{CFT}} .
$$


Recall that conformal invariance constrains the $(n+2)$-point function, $G^{(n+2)}$, of operators of weight $(h, \bar{h})$ to be of the form

$$
G^{(n+2)}\left(w_{1}, \bar{w}_{1} ; \cdots ; w_{n+2}, \bar{w}_{n+2}\right)=\prod_{i<j} w_{i j}^{-2 h /(n+1)} \bar{w}_{i j}^{-2 \bar{h} /(n+1)} Y\left(w_{i j}^{k l} ; \bar{w}_{i j}^{k l}\right)
$$

where $w_{i j}=w_{i}-w_{j}$ and $Y$ is an arbitrary function of the $2((n+2)-3)$ cross ratios, $w_{i j}^{k l}=\frac{w_{i j} w_{k l}}{w_{i l} w_{k j}}, \bar{w}_{i j}^{k l}=\frac{\bar{w}_{i j} \bar{w}_{k l}}{\bar{w}_{i l} \bar{w}_{k j}}$. The case of interest for us is $(h, \bar{h})=(q, 1)$ but we will not yet impose this. We need to integrate over the position of $n$ operators, as in (4.10). To proceed we first use translational invariance to set $w_{n+2}=\bar{w}_{n+2}=0$ and rescale,

$$
w_{i}=\omega_{i} w_{1}, \quad \bar{w}_{i}=\bar{\omega}_{i} w_{1}, \quad i=2, \ldots, n+1
$$

This yields

$$
\begin{aligned}
\int\left(\prod_{i=2}^{n+1} d^{2} w_{i}\right) & G^{(n+2)}\left(w_{1}, \bar{w}_{1} ; \cdots ; w_{n+2}, \bar{w}_{n+2}\right) \\
= & \frac{1}{w_{1}^{2 h+n(h-1)}} \frac{1}{\bar{w}_{1}^{2 \bar{h}+n(\bar{h}-1)}} \int\left(\prod_{i=2}^{n+1} d^{2} \omega_{i}\right) Y\left(w_{i j}^{k l} ; \bar{w}_{i j}^{k l}\right) \\
& \times \prod_{i=2}^{n+1}\left(\omega_{i}^{-2 h /(n+1)}\left(1-\omega_{i}\right)^{-2 h /(n+1)}\right) \prod_{i<j}\left(\omega_{i}-\omega_{j}\right)^{-2 h /(n+1)} \\
& \times \prod_{i=2}^{n+1}\left(\bar{\omega}_{i}^{-2 \bar{h} /(n+1)}\left(1-\bar{\omega}_{i}\right)^{-2 \bar{h} /(n+1)}\right) \prod_{i<j}\left(\bar{\omega}_{i}-\bar{\omega}_{j}\right)^{-2 \bar{h} /(n+1)}
\end{aligned}
$$

Power counting shows that the integrals diverge as $\omega_{i} \sim \omega_{j} \sim 0$ with degree of divergence $(h-1) n$, and similarly there is a divergence when $\bar{\omega}_{i} \sim \bar{\omega}_{j} \sim 0$ with degree of divergence $(\bar{h}-1) n$. When $h=1$ and/or $\bar{h}=1$ the integral has logarithmic divergences.

Specializing to our case we find

$$
\begin{aligned}
\int\left(\prod_{i=2}^{n+1} d^{2} w_{i}\right)\left\langle X_{v \cdots v}\left(w_{1}, \bar{w}_{1}\right)\left(\prod_{i=2}^{n-1} X_{v \cdots v}\left(w_{i}, \bar{w}_{i}\right)\right) X_{v \cdots v}(0,0)\right\rangle_{\mathrm{CFT}} \\
=\left\langle X_{v \cdots v}\left(w_{1}, \bar{w}_{1}\right) X_{v \cdots v}(0,0)\right\rangle_{\mathrm{CFT}} \frac{1}{w_{1}^{(q-1) n}} \tilde{f}\left(\log \left|w_{1}\right|^{2} \mu^{2}\right)
\end{aligned}
$$

where $\tilde{f}\left(\log \left|w_{1}\right|^{2} \mu^{2}\right)$ is the (dimensionless) function that results from the evaluation of the integrals (after appropriate regularization/renormalization) and we explicitly indicate that it will depend on $\log \left|w_{1}\right|^{2} \mu^{2}$ (at most polynomially) because the integrals are logarithmically divergent. We will compute such integrals in the next few subsections.

Fourier transforming and reinstating the coupling $b^{v \cdots v}$ we find

$$
\begin{aligned}
\left\langle X_{v \cdots v}(k, \bar{k})(\right. & \left.\left.\prod_{i=1}^{n} b^{v \cdots v} X_{v \cdots v}(k=0, \bar{k}=0)\right) X_{v \cdots v}(-k,-\bar{k})\right\rangle_{\mathrm{CFT}}= \\
& \left\langle X_{v \cdots v}(k, \bar{k}) X_{v \cdots v}(-k,-\bar{k})\right\rangle_{\mathrm{CFT}}\left(b^{v \cdots v}(k)^{q}\right)^{n} f\left(\log |k| / m^{2}\right) .
\end{aligned}
$$

Wick rotating to Lorentzian signature $k$ becomes $k_{v}$ and this is the identity we wanted to prove. 


\subsection{Deformations of two-dimensional CFTs: TMG example}

In this subsection we consider more explicitly deformations of two dimensional conformal field theories that respect Schrödinger symmetry. In particular, we would like to understand how the spectrum of operators changes as we move from the relativistic fixed point to the Schrödinger fixed point, i.e. we would like to compute the non-relativistic dimension $\Delta_{s}$ of the operator. As in the previous subsection we will use conformal perturbation theory; note that this does not require a weakly coupled realization of the CFT. In contrast to the previous subsection, we will only work to the first non-trivial order. We will, however, explicitly evaluate all integrals. Keeping in mind the example of TMG, we begin with the case where the CFT is deformed by a $(3,1)$ operator $X_{3,1}$ (called $X_{v v}$ in the previous subsection) so that

$$
S_{\mathrm{CFT}} \rightarrow S_{\mathrm{CFT}}-b^{2} \int d^{2} w X_{3,1},
$$

and from here onwards we work in Euclidean signature with $\sqrt{2} u \rightarrow \bar{w}$ and $\sqrt{2} v \rightarrow w$. The numerical factors are included for computational convenience in what follows.

Suppose that the original CFT has primary operators $\mathcal{O}_{h, \bar{h}}$ of dimension $(h, \bar{h})$ (one of which is $\left.X_{(3,1)}\right)$. We would like to compute their dimension in the theory (4.16). Let their two-point functions in the original CFT be normalized as

$$
\left\langle\mathcal{O}_{h, \bar{h}}(w, \bar{w}) \mathcal{O}_{h, \bar{h}}(0,0)\right\rangle=\frac{c_{\mathcal{O}}}{w^{2 h} \bar{w}^{2 \bar{h}}},
$$

where the Euclidean metric is

$$
d s^{2}=d w d \bar{w} .
$$

Now let us calculate the corrections to the two point functions in the deformed theory, working perturbatively in the deformation parameter $b^{2}$. To leading order in $b^{2}$ the corrections to the two point functions are computable from three point functions in the conformal theory

$$
\delta\left\langle\mathcal{O}_{h_{1}, \bar{h}_{1}}(w, \bar{w}) \mathcal{O}_{h_{3}, \bar{h}_{3}}(0,0)\right\rangle=-b^{2} \int d^{2} y\left\langle\mathcal{O}_{h_{1}, \bar{h}_{1}}(w, \bar{w}) X_{3,1}(y, \bar{y}) \mathcal{O}_{h_{3}, \bar{h}_{3}}(0,0)\right\rangle
$$

Note in particular that the correction to the two point function does not in general preserve the orthogonality of the basis; there is operator mixing and the operator basis needs to be diagonalized. For simplicity we will consider here the case of operators which do not mix with other operators.

The three-point functions with the operator $X_{3,1}$ are given by the familiar expression

$$
\begin{aligned}
\left\langle\mathcal{O}_{h_{1}, \bar{h}_{1}}\left(w_{1}, \bar{w}_{1}\right) X_{3,1}\left(w_{2}, \bar{w}_{2}\right) \mathcal{O}_{h_{3}, \bar{h}_{3}}\left(w_{3}, \bar{w}_{3}\right)\right\rangle= & C_{1 X 3} \frac{1}{w_{12}^{h_{1}+3-h_{3}} w_{23}^{3+h_{3}-h_{1}} w_{13}^{h_{3}+h_{1}-3}} \\
& \cdot \frac{1}{\bar{w}_{12}^{\bar{h}_{1}+1-\bar{h}_{3}} \bar{w}_{23}^{1+\bar{h}_{3}-\bar{h}_{1}} \bar{w}_{13}^{\bar{h}_{3}+\bar{h}_{1}-1}},
\end{aligned}
$$

where $w_{i j}=w_{i}-w_{j}$ and $C_{1 X 3}$ are constants. However, there is a subtlety, as this expression holds only at separated points, whilst (4.19) involves an integration which receives contributions from contact terms. This means that (4.20) needs to be replaced by a renormalized 
expression which is well-defined at coincident points. Since the integration is over position space it is convenient to use differential regularization techniques [39] and write down an expression for the three point function which coincides with (4.20) at separate points but is well-defined at coincident points. In $d$ dimensions a distribution $|x|^{-2 \lambda}$ behaves for $x \rightarrow 0$ as:

$$
\frac{1}{|x|^{2 \lambda}} \sim \frac{1}{d+2 n-2 \lambda} \frac{1}{2^{2 n} n !} \frac{\Gamma\left(\frac{d}{2}\right)}{\Gamma\left(\frac{d}{2}+n\right)} S_{d-1} \partial^{2 n} \delta^{(d)}(x),
$$

where $S_{d-1}=2 \pi^{d / 2} / \Gamma(d / 2)$ is the volume of the unit $(d-1)$ sphere. This implies that the distribution has poles at $\lambda=d / 2+n$ where $n=0,1, \cdots$. To obtain a well-defined distribution one subtracts this pole; it suffices to work out the case of $\lambda=d / 2$ since the others can be obtained by differentiation. In particular, in two dimensions one replaces $1 /|x|^{2}$ by

$$
D(x)=\mathcal{R}\left(\frac{1}{|x|^{2}}\right)=\frac{1}{8} \square \ln ^{2}\left(m^{2}|x|^{2}\right)
$$

where $m^{2}$ is the renormalization scale. The renormalized expression differs from $1 /|x|^{2}$ by the infinite term $\delta(x) \log \left(m^{2}|x|^{2}\right)$ localized at $x=0$. This form for the renormalized quantity is specific to two dimensions, and can be obtained taking the $d \rightarrow 2$ limit of the corresponding expression for $d>2$ given in (4.70). The Fourier transform of the renormalized expression was computed in the appendix of [40] and is given by

$$
\tilde{D}(k)=\int d^{2} x e^{-i k x} D(x)=-\pi \ln \left(\frac{k^{2}}{\mu^{2}}\right),
$$

where $\mu^{2}=4 e^{-2 \gamma} m^{2}$.

The three point function of interest has $h_{1}=h_{3}=h$ and $\bar{h}_{1}=\bar{h}_{3}=\bar{h}$, and can be rewritten in the form:

$$
\begin{aligned}
\left\langle\mathcal{O}_{h, \bar{h}}\left(w_{1}, \bar{w}_{1}\right) X_{3,1}\left(w_{2}, \bar{w}_{2}\right) \mathcal{O}_{h, \bar{h}}\left(w_{3}, \bar{w}_{3}\right)\right\rangle= & \frac{C}{4 w_{13}^{2 h-3} \bar{w}_{13}^{2 \bar{h}-1}} \\
& \quad \times \partial_{w_{1}}^{2}\left(\frac{1}{\left|w_{12}\right|^{2}}\right) \partial_{w_{3}}^{2}\left(\frac{1}{\left|w_{23}\right|^{2}}\right) .
\end{aligned}
$$

The structure constant $C$ is given by $C \equiv C_{1 X 1}$. The singularities as $w_{2} \rightarrow w_{1}$ and $w_{2} \rightarrow w_{3}$ are removed via the renormalized expression:

$$
\begin{aligned}
\left\langle\mathcal{O}_{h, \bar{h}}\left(w_{1}, \bar{w}_{1}\right) X_{3,1}\left(w_{2}, \bar{w}_{2}\right) \mathcal{O}_{h, \bar{h}}\left(w_{3}, \bar{w}_{3}\right)\right\rangle= & \frac{C}{4 w_{13}^{2 h-3} \bar{w}_{13}^{2 \bar{h}-1}} \\
& \times \partial_{w_{1}}^{2} \mathcal{R}\left(\frac{1}{\left|w_{12}\right|^{2}}\right) \partial_{w_{3}}^{2} \mathcal{R}\left(\frac{1}{\left|w_{23}\right|^{2}}\right),
\end{aligned}
$$

which manifestly agrees with the previous expression away from contact points. For simplicity we will compute the correction to the two point function at separated points, i.e. we will disregard contact terms as $w_{1} \rightarrow w_{3}$, but it is straightforward to generalize the analysis to include these. Note also that contact terms are in any case absent when the scaling weights are such that $2 h$ and $2 \bar{h}$ are not integral. 
The leading correction to the two point function is:

$$
\delta\left\langle\mathcal{O}_{h, \bar{h}}(w, \bar{w}) \mathcal{O}_{h, \bar{h}}(0,0)\right\rangle=-\frac{b^{2} C}{4 w^{2 h-3} \bar{w}^{2 \bar{h}-1}} \int d^{2} y \partial_{w}^{2} \mathcal{R}\left(\frac{1}{|w-y|^{2}}\right) \partial_{x}^{2} \mathcal{R}\left(\frac{1}{|x-y|^{2}}\right)_{x=0} .
$$

To compute the integral first note that it is a convolution and therefore:

$$
\int d^{2} y \mathcal{R}\left(\frac{1}{|w-y|^{2}}\right) \mathcal{R}\left(\frac{1}{|x-y|^{2}}\right)=\frac{1}{(2 \pi)^{2}} \int d^{2} k e^{i k(w-x)} \tilde{D}^{2}(k),
$$

where the Fourier transform $\tilde{D}(k)$ was given in (4.23). Thus:

$$
\begin{aligned}
\int d^{2} y \mathcal{R}\left(\frac{1}{|w-y|^{2}}\right) \mathcal{R}\left(\frac{1}{|x-y|^{2}}\right) & =\frac{1}{4} \int d^{2} k e^{i k(w-x)} \ln ^{2}\left(k^{2} / \mu^{2}\right), \\
& =2 \pi \frac{1}{|w-x|^{2}} \ln \left(m^{2}|w-x|^{2}\right),
\end{aligned}
$$

where we again use the Fourier transform (4.23). The complete two point function to first order in $b^{2}$ is then:

$$
\left\langle\mathcal{O}_{h, \bar{h}}(w, \bar{w}) \mathcal{O}_{h, \bar{h}}(0,0)\right\rangle=\frac{1}{w^{2 h} \bar{w}^{2 \bar{h}}}\left(c_{\mathcal{O}}-\frac{12 \pi b^{2} C}{w^{2}} \ln \left(\hat{m}^{2}|w|^{2}\right)\right),
$$

where $\hat{m}^{2}=m^{2} e^{-25 / 12}$. This correction respects Schrödinger symmetry and is of the form (4.14) discussed in the previous subsection.

As discussed earlier, it is natural to work with operators of fixed right moving momentum, so we now Fourier transform this expression. For simplicity, we will work out the case $h=\bar{h}$. The general case is a straightforward extension. Recall that the general expression for the Fourier transform of a polynomial in $d$ dimensions is

$$
\int d^{d} x e^{-i \vec{k} \cdot \vec{x}}\left(|x|^{2}\right)^{-\lambda}=\pi^{d / 2} 2^{d-2 \lambda} \frac{\Gamma(d / 2-\lambda)}{\Gamma(\lambda)}\left(|k|^{2}\right)^{\lambda-d / 2},
$$

which is valid when $\lambda \neq(d / 2+n)$, where $n$ is zero or a positive integer. (These are the cases in which the distribution in $x$ is ill-defined, as discussed in (4.21), and one needs to subtract poles.) Differentiating this expression with respect to $\lambda$ results in the identity:

$$
\int d^{d} x e^{-i \vec{k} \cdot \vec{x}}\left(|x|^{2}\right)^{-\lambda} \ln \left(M^{2}|x|^{2}\right)=-\pi^{d / 2} 2^{d-2 \lambda} \frac{\Gamma(d / 2-\lambda)}{\Gamma(\lambda)}\left(|k|^{2}\right)^{\lambda-d / 2} \ln \left(|k|^{2} / \mu^{2}\right),
$$

with

$$
\mu^{2}=4 M^{2} \exp [\psi(d / 2-\lambda)+\psi(\lambda)]
$$

where $\psi(x)$ is the digamma function. In the two-dimensional case, $d^{2} w=1 / 2 d w d \bar{w}$, and applying (anti) holomorphic derivatives leads to further identities such as:

$$
\int d w d \bar{w} e^{-i k w-i \bar{k} \bar{w}} w^{-2}|w|^{-2 \lambda}=-\pi 2^{3-2 \lambda} \frac{\Gamma(1-\lambda)}{\Gamma(\lambda+2)} k^{2}|k|^{2(\lambda-1)},
$$

where $|k|^{2}=4 k \bar{k}$. After differentiating with respect to $\lambda$ one obtains:

$$
\int d w d \bar{w} e^{-i k w-i \bar{k} \bar{w}} w^{-2}|w|^{-2 \lambda} \ln \left(M^{2}|w|^{2}\right)=\pi 2^{3-2 \lambda} \frac{\Gamma(1-\lambda)}{\Gamma(\lambda+2)} k^{2}|k|^{2(\lambda-1)} \ln \left(|k|^{2} / \mu_{1}^{2}\right),
$$


where $\mu_{1}^{2}=4 M^{2} \exp [\psi(1-\lambda)+\psi(2+\lambda)]$.

Using these identities the Fourier transform of the corrected two point function of a scalar is:

$$
\left\langle\mathcal{O}_{h, h}(k, \bar{k}) \mathcal{O}_{h, h}(-k,-\bar{k})\right\rangle=A k^{2 h-1} \bar{k}^{2 h-1}\left(1+B k^{2} \ln \left(|k|^{2} / \mu_{1}^{2}\right)\right)
$$

where

$$
A=c_{\mathcal{O}} \pi 2^{2-4 h} \frac{\Gamma(1-2 h)}{\Gamma(2 h)} ; \quad B=-\frac{6 \pi C}{c_{\mathcal{O}} h(2 h+1)} b^{2} .
$$

Note that the normalization of scalar operators in the CFT required to match the standard holographic normalization is [12]:

$$
c_{\mathcal{O}}=(2 h-1) \frac{\Gamma(2 h)}{\pi \Gamma(2 h-1)}
$$

in other words, a canonically normalized bulk scalar field will lead to two point functions in the CFT with this normalization. In momentum space the general expression for the two point function in a Schrödinger invariant theory takes the form:

$$
\left\langle\mathcal{O}_{\Delta_{s}}(k, \bar{k}) \mathcal{O}_{\Delta_{s}}(-k,-\bar{k})\right\rangle=\tilde{g}(k) \bar{k}^{\Delta_{s}-1},
$$

The corrected two point function is consistent with this form where at leading order in $b^{2}$

$$
\begin{aligned}
\Delta_{s} & =2 \bar{h}+\gamma(k) ; \quad \gamma(k)=-\frac{6 \pi C}{c_{\mathcal{O}} h(2 h+1)} b^{2} k^{2} ; \\
\tilde{g}(k) & =-2^{3-4 h} \frac{\Gamma(2-2 h)}{\Gamma(2 h-1)} k^{\Delta_{s}-1},
\end{aligned}
$$

and we have used the normalization (4.37) in the latter equality. The normalization factor $\tilde{g}(k)$ is written in this way in anticipation of the holographic result in section 6 , which indeed takes this form to all orders in $b$. As anticipated, the anomalous dimension depends explicitly on the holomorphic momentum, and vanishes when this momentum is zero. Note that on general grounds it is also clear that correlation functions in these theories should depend on the combination $b^{2} k^{2}$, as this is the quantity which is invariant under rescalings of the bulk lightcone coordinates. In other words, by rescalings of the lightcone coordinates one can rescale $b^{2}$ to any non-zero value but $b^{2} k^{2}$ is independent of such rescalings.

Analogous corrections would be expected for the two point functions of all operators, but in general, as mentioned above, operators will mix and one will need to rediagonalize the basis of operators. The leading order corrections are all determined by the three point function coefficients $C_{1 X 3}$. This means in particular that, because the form of the OPE for the stress energy tensor $(T, \bar{T})$ in a conformal field theory implies that the stress energy tensor does not have a three point function with the operator $X_{3,1}$, the correlation functions of $(T, \bar{T})$ are not corrected at order $b^{2}$. This is as one might have expected, since the stress energy tensor should not acquire an anomalous dimension. However, as we will discuss shortly, the conserved stress energy tensor of a non-relativistic theory is necessarily not symmetric, and thus the operators $(T, \bar{T})$ are not natural operators in the deformed theory. 
As discussed earlier, the deforming operator $X$ itself generally acquires an anomalous dimension when its lightcone momentum $k_{v}$ is non-zero. The leading correction to the two point function is given by (4.29), and generically corrections to the two point functions occur at all orders $b^{2 n}$, and are related to $(n+2)$-point functions in the CFT. The holographic computation in section 7 indicates that this series in $b^{2}$ can be resummed into the simple form:

$$
\langle X(k) X(-k)\rangle_{b}=\tilde{g}\left(k_{v}\right) k_{u}^{\Delta_{s}-1},
$$

where the normalization $\tilde{g}\left(k_{v}\right)$ is lightcone momentum dependent and so is the non-relativistic scaling dimension, $\Delta_{s}=1+\sqrt{1+b^{2} k_{v}^{2}}$. Notice in particular the scaling dimension is larger than two, for $b^{2}>0$ and non-zero $k_{v}$, and thus the operator is irrelevant. This expression is completely analogous to the expression for the probe scalar operator (4.38). Expanding this expression perturbatively in $b^{2}$ results in:

$$
\langle X(k) X(-k)\rangle_{b}=\tilde{g}\left(k_{v}\right) k_{u}\left(1+\left(\frac{1}{2} b^{2} k_{v}^{2}-\frac{b^{4} k_{v}^{4}}{8}\right) \ln \left(k_{u}\right)+\frac{b^{4} k_{v}^{4}}{8}\left(\ln \left(k_{u}\right)\right)^{2}+\cdots\right)
$$

to order $b^{4}$. It would be interesting to see whether the terms at order $b^{4}$ follow from generic features of the four-point function of $X$, or whether these coefficients are specific to the theory dual to TMG.

\subsection{Other deformations of 2d CFTs: general $z$}

Let us next consider the more general situation in which one deforms a $2 \mathrm{~d}$ CFT by a $(p, q)$ operator $\mathcal{Y}_{p, q}$ where $(p, q)$ are the CFT scaling weights corresponding to $(v, u)$ respectively,

$$
S_{\mathrm{CFT}} \rightarrow S_{\mathrm{CFT}}+b_{p, q} \int d u d v \mathcal{Y}_{p, q}
$$

Such a deformation will respect non-relativistic scale invariance with dynamical exponent $z$ under which $u \rightarrow \lambda^{z} u$ and $v \rightarrow \lambda^{2-z} v$ provided that

$$
(p-1)(z-2)=(q-1) z
$$

For $z \neq 2$ scale invariance is respected provided that $q=1$ with $p$ arbitrary. For $z \geq 2$ the condition can be satisfied for discrete values of the weights $(p, q)$. Note that the ratio $u^{2-z} v^{-z}$ is scale invariant, whilst $(u v)$ scales with dimension two (as in the relativistic theory). As a simple example in the case of $z=3$ a classical action one can write down is:

$$
S=\int d u d v\left(\partial_{u} \Phi\right)\left(\partial_{v} \Phi\right)\left(1+b_{4,2}\left(\partial_{u} \Phi\right)\left(\partial_{v} \Phi\right)^{3}\right)
$$

where $b_{2,4}$ characterizes the deformation of the original relativistic CFT by a dimension $(2,4)$ operator. Dual holographic geometries which respect such non-relativistic scale invariance with a generic dynamical exponent $z \neq 1$ are given by:

$$
d s^{2}=\frac{d r^{2}}{r^{2}}+\frac{1}{r^{2}}\left(2 d u d v-b^{2} \frac{d u^{2}}{r^{2(z-1)}}\right) .
$$


The symmetry group consists of:

$$
\begin{aligned}
& \mathcal{H}: u \rightarrow u+a, \quad \mathcal{M}: v \rightarrow v+a, \\
& \mathcal{D}: r \rightarrow(1-a) r, \quad u \rightarrow(1-z a) u, \quad v \rightarrow(1+(z-2) a) v,
\end{aligned}
$$

and thus the $v$ coordinate scales non-trivially except when $z=2$. Note that for $z>2$ the coordinate $v$ scales as a negative power of the dilatation.

For generic $z$ such non-relativistic backgrounds can be straightforwardly realized as solutions of Einstein gravity coupled to massive vectors, although much of the literature has concentrated on the Schrödinger case for which $z=2$. The case of critical speeding up, namely $z<1$, for which $b^{2}<0$, will be explored in detail in other work [41]; in this case the spacetime is asymptotically anti-de Sitter and many of the conceptual subtleties of the $z>1$ case are absent. Spacetimes with generic $z$ can also be realized as solutions of TMG: the metric (4.45) solves the TMG field equations when $\mu=(2 z-1)$. These TMG solutions were discussed in [42] and fit into the classification given in [43] as pp-waves. For $b>0$, these solutions with $u$ compactified were recently discussed in [44].

Now consider operators $\mathcal{O}_{h, \bar{h}}$ of conformal dimensions $(h, \bar{h})$ in the original CFT in Euclidean signature with $\sqrt{2}(v, u) \rightarrow(w, \bar{w})$. Under the non-relativistic scaling symmetry with exponent $z$ such that $w \rightarrow \lambda^{2-z}, \bar{w} \rightarrow \lambda^{z} \bar{w}$, this operator scales as:

$$
\mathcal{O}_{h, \bar{h}}(w, \bar{w}) \rightarrow \lambda^{-h(2-z)-\bar{h} z} \mathcal{O}_{h, \bar{h}}\left(\lambda^{2-z} w, \lambda^{z} \bar{w}\right),
$$

and thus the non-relativistic scaling dimension is

$$
\Delta_{\mathrm{nr}}=h(2-z)+\bar{h} z .
$$

Non-relativistic scale invariance constrains the two point functions to be of the form

$$
\left\langle\mathcal{O}_{\Delta_{\mathrm{nr}}}(w, \bar{w}) \mathcal{O}_{\Delta_{\mathrm{nr}}^{\prime}}(0,0)\right\rangle=\frac{1}{\bar{w}^{\left(\Delta_{\mathrm{nr}}+\Delta_{\mathrm{nr}}^{\prime}\right) / z}} f(\mathcal{X}),
$$

where $f(\mathcal{X})$ is an arbitrary function of the scale invariant quantity

$$
\mathcal{X}=w^{-z} \bar{w}^{2-z} .
$$

Note that for general $z$ operators of different scaling dimension are not precluded from having a non-zero two point function, although when $z=2$ the additional special conformal symmetry ensures that the correlator is only non-zero when $\Delta_{\mathrm{nr}}=\Delta_{\mathrm{nr}}^{\prime}$.

Consider now the 2-point function (4.17) of an operator $\mathcal{O}_{h, \bar{h}}$ in a relativistic CFT. A simple computation shows that it can be written in the form (4.49) with the non-relativistic dimension $\Delta_{\mathrm{nr}}=\Delta_{\mathrm{nr}}^{\prime}$ given in (4.48) and $f(\mathcal{X})=c_{\mathcal{O}} \mathcal{X}^{2 h / z}$. Using conformal perturbation theory, the leading correction to this two point function is given by

$$
\delta\left\langle\mathcal{O}_{h, \bar{h}}(w) \mathcal{O}_{h, \bar{h}}(0)\right\rangle \sim \frac{b_{p, q}}{w^{2 h-p} \bar{w}^{2 \bar{h}-q}} \int d^{2} y\left(\frac{1}{(w-y)^{p} y^{p}(\bar{w}-\bar{y})^{q} \bar{y}^{q}}\right) .
$$

Consider the case where the deforming operator $\mathcal{Y}_{p, q}$ has integral spin, i.e. $p=q+n$ with $n$ an integer. Then the (renormalized) correction can be written as:

$$
b_{p, q} \frac{1}{w^{2 h-q-n} \bar{w}^{2 \bar{h}-q}}(-1)^{n} \frac{\Gamma(q)^{2}}{\Gamma(q+n)^{2}} \partial_{w}^{2 n} \int d^{2} y \mathcal{R}\left(\frac{1}{|y-w|^{2 q}}\right) \mathcal{R}\left(\frac{1}{|y|^{2 q}}\right) .
$$


When $q$ is not an integer computing the integral leads to:

$$
(-1)^{n+1} \pi \frac{\Gamma(1-q)^{2} \Gamma(2 q+2 n-1)}{\Gamma(q+n)^{2} \Gamma(1-2 q)} b_{p, q} \frac{1}{w^{2 h+n+p-1} \bar{w}^{2 \bar{h}+p-1}} .
$$

This implies that the two point function to first order in the deformation can be written as:

$$
\begin{aligned}
\left\langle\mathcal{O}_{h, \bar{h}}(w, \bar{w}) \mathcal{O}_{h, \bar{h}}(0,0)\right\rangle & =\frac{1}{w^{2 h} \bar{w}^{2 \bar{h}}}\left(c_{\mathcal{O}}+\alpha b_{p, q} w^{1-p} \bar{w}^{1-q}\right), \\
& =\frac{1}{w^{2 h} \bar{w}^{2 h}}\left(c_{\mathcal{O}}+\alpha b_{p, q} \mathcal{X}^{(p-1) / z}\right),
\end{aligned}
$$

where $\alpha$ is a numerical constant and (4.43) is used in the last equality. Thus, the two point function indeed preserves non-relativistic scale invariance at this order, with the two point function being of the form (4.49) with the same values of $\Delta_{\mathrm{nr}}=\Delta_{\mathrm{nr}}^{\prime}$ (given in (4.48)) and

$$
f(\mathcal{X})=\mathcal{X}^{2 h / z}\left(c_{\mathcal{O}}+\alpha b_{p, q} \mathcal{X}^{(p-1) / z}\right)
$$

One can also understand why the corrections must behave as $b_{p, q} w^{1-p} \bar{w}^{1-q}$ as follows: the deformed action will remain invariant under the original dilatation symmetries provided that the coupling $b_{p, q}$ is also transformed. In particular, under the scaling $\bar{z} \rightarrow \bar{\lambda} \bar{z}$ the deformation to the action is invariant provided that $b_{p, q} \rightarrow \bar{\lambda}^{q-1} b_{p, q}$. Since the corrections to the correlation functions respect this symmetry they must be organized in powers of $b_{p, q} w^{1-p} \bar{w}^{1-q}$.

For integral $q$ the analysis is more complicated as we need to use the renormalized expressions for the distributions, and we obtain instead for the integral:

$$
(-1)^{n} 2 \pi \frac{\Gamma(q)^{2}}{\Gamma(q+n)^{2}} b_{p, q} \partial_{w}^{2 n} \square_{w}^{2 q-2}\left(|w|^{-2} \ln \left(m^{2}|w|^{2}\right)\right),
$$

which gives a correction to the two point function proportional to:

$$
b_{p, q} \frac{1}{w^{2 h+(p-1)} \bar{w}^{2 \bar{h}+(q-1)}} \ln \left(\tilde{m}^{2}|w|^{2}\right),
$$

with $\tilde{m}^{2}$ a rescaled mass scale. This implies that the two point function to first order in the deformation can be written as:

$$
\begin{aligned}
\left\langle\mathcal{O}_{h, \bar{h}}(w, \bar{w}) \mathcal{O}_{h, \bar{h}}(0,0)\right\rangle & =\frac{1}{w^{2 h} \bar{w}^{2 \bar{h}}}\left(c_{\mathcal{O}}+\beta b_{p, q} w^{(1-p)} \bar{w}^{(1-q)} \ln \left(\tilde{m}^{2}|w|^{2}\right)\right), \\
& =\frac{1}{w^{2 h} \bar{w}^{2 \bar{h}}}\left(c_{\mathcal{O}}+\beta b_{p, q} \mathcal{X}^{(p-1) / z} \ln \left(\tilde{m}^{2}|w|^{2}\right)\right),
\end{aligned}
$$

where $\beta$ is a computable numerical constant and (4.43) is used in the last equality. Note that the correction to the two point function preserves the $z=2$ non-relativistic scale invariance when $q=1$ for any $p$. In this case not only $f(\mathcal{X})$ but also the non-relativistic dimension of the operator get corrections and the 2-point function is given by (4.49) with

$$
\begin{aligned}
f(\mathcal{X}) & =\mathcal{X}^{-2 h / z}\left(c_{\mathcal{O}}+\beta b_{p, q} \mathcal{X}^{(p-1) / z} \ln \left(\tilde{m}^{2} \mathcal{X}^{-1 / z}\right)\right)+\mathcal{O}\left(b_{p, q}^{2}\right) ; \\
\Delta_{\mathrm{nr}}=\Delta_{\mathrm{nr}}^{\prime} & =h(2-z)+\bar{h} z-\beta b_{p, q} \mathcal{X}^{(p-1) / z}+\mathcal{O}\left(b_{p, q}^{2}\right) .
\end{aligned}
$$

When $z=2$, namely the deformation is by a $(p, 1)$ operator, the scale invariant quantity $\mathcal{X}$ depends only on the coordinate $v$ and one can check that these formulas reduce to the ones we presented in the previous subsection. 


\subsection{Massive vector model with $z=2$}

The specific case of a deformation by a $(2,1)$ operator corresponds to the $z=2$ massive vector model. The above considerations indicate that generically under such a deformation operators will acquire anomalous dimensions depending on the lightcone momentum as functions of $b_{2,1} k_{v}$.

In the bulk calculations in later sections, we will focus on scalar operators dual to minimally coupled scalar fields, as well as the operators dual to the bulk metric and massive vector field. We indeed find that such scalar operators and the vector operators acquire anomalous dimensions, and that these anomalous dimensions are functions of $\left(b_{2,1} k_{v}\right)^{2}$. We can understand the latter straightforwardly as follows. If one considers scalar operators dual to minimally coupled scalar fields in the bulk, the three point function between two scalar operators and the vector operator necessarily vanishes. It vanishes because minimally coupled implies that there is no three point coupling ${ }^{4}$ between the bulk scalar fields and vector field: the bulk action under consideration is

$$
S=\int d^{d+1} x \sqrt{-g}\left(R-2 \Lambda-\frac{1}{4} F^{2}-\frac{1}{2} m^{2} A^{2}-\frac{1}{2}(\partial \Phi)^{2}-\frac{1}{2} m_{\Phi}^{2} \Phi^{2}\right) .
$$

Expanding the field equations to cubic order in fluctuations couples the scalar to the vectors via the metric, and thus there is generically a non-trivial four point function between two scalars and two vectors. The first correction to the scalar two point function therefore follows from this four point function between two scalar operators and two insertions of the vector operator. The leading correction will hence occur at order $\left(b_{2,1} k_{v}\right)^{2}$, but to compute it via conformal perturbation theory we would need to know the explicit form of the four point function (since it is of course not completely determined by conformal invariance). The bulk action implies that only correlation functions involving two scalars and an even number of vectors is not zero, and therefore subsequent corrections should be organized in powers of $\left(b_{2,1} k_{v}\right)^{2}$. Indeed this feature is seen in the holographic dual in section 6 , along with the stronger result that the anomalous dimension resums into a closed form. It would be interesting to derive the latter result from a null dipole realization.

A similar story holds for the deforming vector operator itself: the action implies there is no cubic coupling between three vectors, and thus the three point function between three vector operators is zero. Since the bulk action (4.60) is quadratic in the massive vector fields, only correlators with an even number of vector operators are non-zero, and thus that the corrections to the vector two point function in the deformed theory should be organized in powers of $\left(b_{2,1} k_{v}\right)^{2}$.

Let us now consider the scaling dimensions of the vector operator (in $d=2$ ). Consider first the theory at $b_{2,1}=0$. The mass of the bulk vector field is such that it corresponds to a vector operator of dimension three in the dual conformal field theory. Splitting the vector operator into holomorphic and anti-holomorphic components, one sees that there is

\footnotetext{
${ }^{4}$ Strictly speaking, the vanishing of the bulk three point coupling does not always guarantee vanishing of the corresponding three point function, see [45, 46]. The boundary counterterms required by holographic renormalization can induce non-zero three point functions even when the bulk coupling is zero; this happens for extremal correlators, but none of the correlators discussed here is extremal.
} 
a $(2,1)$ operator $X_{v}$ and a $(1,2)$ operator $X_{u}$. Since in the conformal field theory the two point functions are:

$$
\left\langle X_{v}(w, \bar{w}) X_{v}(0,0)\right\rangle=\frac{c_{\mathcal{X}}}{w^{4} \bar{w}^{2}} ; \quad\left\langle X_{u}(w, \bar{w}) X_{u}(0,0)\right\rangle=\frac{c_{\mathcal{X}}}{w^{2} \bar{w}^{4}},
$$

with $c_{\mathcal{X}}$ normalizations, the scaling dimensions with respect to the Schrödinger symmetry are:

$$
\Delta_{s}\left(X_{v}\right)=2 ; \quad \Delta_{s}\left(X_{u}\right)=4 .
$$

This is in agreement with the non-relativistic scaling dimensions of the source and vev coefficients found holographically in (8.31). Switching on the deformation by the operator $X_{v}$, we expect that the scaling dimensions of both $X_{u}$ and $X_{v}$ at non-zero $k_{v}$ are corrected, with the corrections being organized in powers of $\left(b_{2,1} k_{v}\right)^{2}$. The holographic calculation of section 8 gives explicit expressions for these scaling dimensions at finite $b_{2,1}$. Just as in the case of TMG, the dimensions are expressed as a square root, with the dimensions of both operators being greater than two, at non-zero $k_{v}$.

\subsection{Higher spacetime dimensions}

There has been considerable interest in using massive vector models in dimensions higher than three to model non-relativistic theories. As explained in subsection 3.1, to leading order in $b$ the standard AdS/CFT dictionary implies that switching on $b$ corresponds to switching on a source for a vector operator $X^{a}$ of dimension

$$
\Delta_{v}=d+z-1
$$

in the $d$-dimensional dual CFT, i.e.

$$
S_{\mathrm{CFT}} \rightarrow S_{\mathrm{CFT}}+\int d^{d} x b X_{v}
$$

Corresponding type IIB and eleven-dimensional supergravity solutions for general $z$ are given in [30]. In this section we will focus on the case of $z=2$ in dimension $d$, and discuss the leading corrections to operator correlation functions in the deformed theory. As already mentioned, the leading corrections to the deforming operator itself and to scalar operators dual to minimally coupled scalar fields occur at order $b^{2}$, and are derived from four-point functions in the conformal theory.

Corrections at order $b$ can however occur for complex scalar operators as these can have non-zero three point functions with the deforming vector operator. Let us consider a complex scalar operator $\mathcal{O}$ with dimension $\Delta$ in the original conformal field theory. It has a two point function in the conformal field theory given by

$$
\langle\overline{\mathcal{O}}(x) \mathcal{O}(0)\rangle=\frac{c_{\Delta}}{|x|^{2 \Delta}},
$$

where $c_{\Delta}$ is the operator normalization and we are working in Euclidean signature. Noticing that

$$
|x|^{2}=w \bar{w}+x^{i} x_{i}
$$


we see that the operators are also primary from the perspective of Schrödinger symmetry: under $\bar{w} \rightarrow \lambda^{2} \bar{w}, x^{i} \rightarrow \lambda x^{i}$ with $w$ invariant, the operators scale with non-relativistic scaling dimension $\Delta_{s}=\Delta$.

Next consider the correction at first order in $b$ to this two point function in the deformed theory (4.64). The leading correction to the two point function is:

$$
\langle\overline{\mathcal{O}}(x) \mathcal{O}(0)\rangle=b \int d^{d} y\left\langle\overline{\mathcal{O}}(x) X_{v}(y) \mathcal{O}(0)\right\rangle .
$$

This can be computed by first noting that three point functions between a complex scalar and a vector are fixed by conformal invariance to have the form (derived holographically in $[47])$ :

$$
\left\langle\overline{\mathcal{O}}(x) X^{\mu}(y) \mathcal{O}(z)\right\rangle=\frac{i C}{|x-z|^{2 \Delta+1-\Delta_{v}}|x-y|^{\Delta_{v}-1}|y-z|^{\Delta_{v}-1}}\left(\frac{(x-y)^{\mu}}{|x-y|^{2}}-\frac{(z-y)^{\mu}}{|z-y|^{2}}\right),
$$

where $C$ is a (real) normalization factor.

As in the previous sections, the expression for the three point function needs to be regularized to take into account contact term contributions to the integral. In the case of $z=2$ the deforming vector operator has dimension $\Delta_{v}=d+1$. Using this fact, the three point function can be rewritten as:

$$
\left\langle\overline{\mathcal{O}}(x) X^{\mu}(y) \mathcal{O}(0)\right\rangle=-\frac{i C}{d|x|^{2 \Delta-d}}\left(\frac{1}{|y|^{d}} \partial_{x_{\mu}} \frac{1}{|x-y|^{d}}+\frac{1}{|x-y|^{d}} \partial_{y_{\mu}} \frac{1}{|y|^{d}}\right) .
$$

In differential regularization one replaces $1 /|x|^{d}$ by the well-defined expression:

$$
\mathcal{R}\left(\frac{1}{|x|^{d}}\right)=-\frac{1}{2(d-2)} \square \frac{1}{|x|^{d-2}}\left(\ln \left(m^{2}|x|^{2}\right)+\frac{2}{d-2}\right),
$$

which is the generalization of (4.22) to arbitrary dimensions $d>2$. (The last term in (4.70) is scheme dependent.) Then the leading correction to the two point function is:

$$
\delta\langle\overline{\mathcal{O}}(x) \mathcal{O}(0)\rangle=-\frac{2 b}{d|x|^{2 \Delta-d}} i C \partial_{w} \int d^{d} y \mathcal{R}\left(\frac{1}{|y|^{d}}\right) \mathcal{R}\left(\frac{1}{|x-y|^{d}}\right) .
$$

The integral can be computed analogously to the two dimensional case via the convolution:

$$
\int d^{d} y \mathcal{R}\left(\frac{1}{|y|^{d}}\right) \mathcal{R}\left(\frac{1}{|x-y|^{d}}\right)=\frac{1}{(2 \pi)^{d}} \int d^{d} k e^{i \vec{k} \cdot \vec{x}} \tilde{D}_{d}^{2}(k),
$$

where

$$
\tilde{D}_{d}(k)=\int d^{d} x e^{-i \vec{k} \cdot \vec{x}} \mathcal{R}\left(\frac{1}{|x|^{d}}\right)=-\frac{2 \pi^{d / 2}}{(d-2) \Gamma(d / 2-1)} \ln \left(|k|^{2} / \mu^{2}\right),
$$

with $\mu^{2}=4 m^{2} \exp (\Psi(1)+\Psi(d / 2-1))$. Note that in $d=4$ this reduces to $\mu=2 \mathrm{~m} / \gamma^{\prime}$ since the Euler constant $\gamma^{\prime}=\exp (-\Psi(1))=\exp (\gamma)$. To derive this formula, one can use the expansion of the identity (4.30) with $2 \lambda=d-2-2 a$ :

$$
\int d^{d} x e^{-i \vec{k} \cdot \vec{x}} \frac{1}{|x|^{d-2}}(m|x|)^{2 a}=\pi^{d / 2} 2^{2+2 a} \frac{\Gamma(1+a)}{\Gamma(d / 2-1+a)}|k|^{-2}(|k| / m)^{-2 a}
$$


in powers of $a$. Equating terms at each order in $a$ results in the identities:

$$
\begin{aligned}
\int d^{d} x e^{-i \vec{k} \cdot \vec{x}} \frac{1}{|x|^{d-2}} & =\frac{4 \pi^{d / 2}}{|k|^{2} \Gamma(d / 2-1)} ; \\
\int d^{d} x e^{-i \vec{k} \cdot \vec{x}} \frac{\ln \left(m^{2} x^{2}\right)}{|x|^{d-2}} & =-\frac{4 \pi^{d / 2}}{|k|^{2} \Gamma(d / 2-1)} \ln \left(|k|^{2} / \mu^{2}\right) ; \\
\int d^{d} x e^{-i \vec{k} \cdot \vec{x}} \frac{\ln ^{2}\left(m^{2}|x|^{2}\right)}{|x|^{d-2}} & =\frac{4 \pi^{d / 2}}{|k|^{2} \Gamma(d / 2-1)}\left(\ln ^{2}\left(|k|^{2} / \mu^{2}\right)+\frac{\pi^{2}}{6}-\Psi^{(1)}(d / 2-1)\right),
\end{aligned}
$$

where $\Psi^{(m)}(x)$ is the polygamma function, the $(m+1)$-th derivative of the gamma function. These identities generalize similar ones for $d=4$ derived in [39].

Using the third of these identities we note that

$$
\begin{aligned}
\int d^{d} y \mathcal{R}\left(\frac{1}{|y|^{d}}\right) \mathcal{R}\left(\frac{1}{|x-y|^{d}}\right) \\
=-\frac{\pi^{d / 2}}{(d-2)^{2} \Gamma(d / 2-1)} \square \frac{1}{|x|^{d-2}}\left(\ln ^{2}\left(m^{2}|x|^{2}\right)-\frac{\pi^{2}}{6}+\Psi^{(1)}(d / 2-1)\right) .
\end{aligned}
$$

Note however that away from $x=0$,

$$
\square\left(\frac{\ln ^{2}\left(m^{2}|x|^{2}\right)}{|x|^{d-2}}\right)=\frac{1}{|x|^{d}}\left(4(d-2) \ln \left(m^{2}|x|^{2}\right)+8(3-d)\right) ，
$$

and therefore the leading correction to the two point function has the form:

$$
\langle\overline{\mathcal{O}}(x) \mathcal{O}(0)\rangle=\frac{c_{\Delta}}{\left(x^{2}\right)^{\Delta}}+i c_{1} \frac{b}{|x|^{2 \Delta-d}} \partial_{w}\left[\mathcal{R}\left(\frac{\ln \left(M^{2}|x|^{2}\right)}{|x|^{d}}\right)\right],
$$

where $M$ is a rescaled mass scale and $c_{1}$ is a real numerical constant proportional to the three point function constant $C$ :

$$
c_{1}=\frac{8 \pi^{d / 2}}{d(d-2) \Gamma(d / 2-1)} C .
$$

The corrected two point function can be rewritten as:

$$
\langle\overline{\mathcal{O}}(x) \mathcal{O}(0)\rangle=\frac{c_{\Delta}}{|x|^{2 \Delta}}+i c_{1} b \frac{d}{2 \Delta} \partial_{w}\left[\mathcal{R}\left(\frac{\ln \left(\tilde{M}^{2}|x|^{2}\right)}{|x|^{2 \Delta}}\right)\right],
$$

where $\tilde{M}^{2}=M^{2} \exp (1 / \Delta-2 / d)$. In the case where $\Delta$ is non-integral, the Fourier transform is:

$$
\langle\overline{\mathcal{O}}(k) \mathcal{O}(-k)\rangle=c_{\Delta} \pi^{d / 2} 2^{d-2 \Delta} \frac{\Gamma(d / 2-\Delta)}{\Gamma(\Delta)}|k|^{2 \Delta-d}\left(1+\frac{d}{2 \Delta} k b c_{1} \ln \left(|k|^{2} / \tilde{\mu}^{2}\right)\right),
$$

where $k$ is the momentum conjugate to $w$. (When $\Delta$ is integral we need to Fourier transform the renormalized expression, which can be done analogously to previous sections.) Analytically continuing back to Lorentzian signature, the time-ordered correlator is

$$
\langle\mathcal{T} \overline{\mathcal{O}}(k) \mathcal{O}(-k)\rangle=c\left(2 k_{u} k_{v}+k_{i} k^{i}-i \epsilon\right)^{\Delta_{s}-d / 2},
$$


where $c=c_{\Delta} \pi^{d / 2} 2^{d-2 \Delta} \Gamma(d / 2-\Delta) / \Gamma(\Delta)$ in the renormalization scheme where $\tilde{\mu}^{2}=1$. The scaling dimension $\Delta_{s}$ is to leading order in $b$ given by:

$$
\Delta_{s}=\Delta+\gamma, \quad \gamma=\frac{1}{\sqrt{2}}\left(b k_{v}\right) \frac{4 \pi^{d / 2} C}{\Delta(d-2) \Gamma(d / 2-1)}
$$

and the anomalous dimension hence depends on $\left(b k_{v}\right)$.

To describe the theory at finite $b$ the null dipole picture should be of use: in the context of the $S^{5}$ reduction of type IIB, the dual theory should be the null dipole deformation of $\mathcal{N}=4 \mathrm{SYM}$. In this case the global $R$ symmetry charges are used to determine the dipole vectors of the various fields as follows. Choose a constant element $B \in s u(4)$, where $s u(4)$ is the Lie algebra of SU(4). Then denote the elements of $B$ in the 4 representation as $U_{j \bar{k}}$, where $j, \bar{k}=1, \cdots, 4$, and denote the elements of $B$ in the $\mathbf{6}$ representation as the antisymmetric matrices $M_{I J}$, where $I, J=1, \cdots, 6$. Let $u_{I}^{l}$ be an eigenvector of $M_{I J}$ with eigenvalue $L_{l}$. The $\mathcal{N}=4 \mathrm{SYM}$ complex valued scalar fields $\Phi^{l}=\sum_{I} u_{I}^{l} \Phi^{I}$ are then assigned (null) dipole vectors $L_{l}$. Similarly the fermionic fields are assigned dipole vectors determined by the eigenvalues of the matrix $U_{j \bar{k}}$.

Suppose for example one chooses $M_{I J}$ such that only $M_{12}=-M_{21}$ is non-zero. Next complexify the six scalars into three complex scalars $\Phi_{c}^{a}$, with $a=1,2,3$ where $\Phi_{c}^{1}=$ $\Phi^{1}+i \Phi_{2}$ and so on. Then $\Phi_{c}^{1}$ has non-zero dipole charge, given by $L_{\mu}=-i b \delta_{\mu u}$, while the other two complex scalars have zero charge. The dipole product (2.14) can be expanded to leading order in $b$ as:

$$
\Phi_{c}^{1} * \Phi_{c}^{1 \dagger}=\Phi_{1}^{2}+\Phi_{2}^{2}+b\left(\partial_{v} \Phi_{1} \Phi_{2}-\partial_{v} \Phi_{2} \Phi_{1}\right)+\cdots
$$

The analogue of the Seiberg-Witten map in this case relates fields $\Phi$ in the dipole theory to fields $\Phi_{o}$ in the ordinary theory as:

$$
\Phi=\Phi_{o} \mathcal{P}\left(\exp \left(i L^{\mu} \int_{0}^{1} A_{\mu}(x+t L) d t\right)\right)
$$

Substituting into the $\mathcal{N}=4 \mathrm{SYM}$ action and expanding to leading order in $b_{u}$ results in:

$$
S=S_{\mathcal{N}=4}+\int d^{4} x b \mathcal{V}_{v}^{12}+\cdots
$$

where the vector operator $V_{\mu}^{I J}$ transforms as the $\mathbf{1 5}$ of the $\mathrm{SO}(6) \mathrm{R}$ symmetry group and has the form:

$$
\mathcal{V}_{\mu}^{[I J]}=\operatorname{Tr}\left(F_{\mu \nu} \phi^{[I} D^{\nu} \phi^{J]}+\sum_{K} \phi^{K} D_{\mu}\left(\phi^{[K} \phi^{I} \phi^{J]}\right)+\cdots\right) .
$$

Here $I, J=1, \cdots, 6$ are $\mathrm{R}$ symmetry indices, $D_{\mu}$ is the covariant derivative with respect to gauge fields $A_{\mu}$ of the field strength $F_{\mu \nu}$ and square parentheses denote antisymmetrisation. The ellipses denote fermionic terms. At linear order in $b$ the null dipole theory is indeed equivalent to a deformation of $\mathcal{N}=4$ SYM by a constant null source $b$ for an operator of relativistic dimension $(d+1)=5$. This operator however has Schrödinger dimension 
four, using the fact that $\Delta_{s}\left(A_{v}\right)=0$ whilst $\Delta_{s}\left(\phi^{I}\right)=\Delta_{s}\left(A_{u}\right)=\Delta_{s}\left(A_{i}\right)=1$. Expanding to higher order in $b$ will lead to a series of deformations respecting Schrödinger symmetry. Note that the amount of supersymmetry preserved by the dipole deformation depends on the explicit choice of $M_{I J}$.

It is important to note that all these deformations are marginal with respect to the Schrödinger symmetry. This immediately follows from the fact that every term in the null dipole product of any two fields has the same Schrödinger dimension, even though the relativistic conformal dimensions of the terms are different. One can see this property in (4.83), since a term at order $b^{n}$ involves $n$ derivatives in the $v$ direction, which leaves the Schrödinger dimension unchanged but increases the usual dimension. More generally, the product of any two fields $\left(\Phi_{q_{1}}, \Phi_{q_{2}}\right)$ with dipole charges $L_{1}=-i n_{1} b$ and $L_{2}=-i n_{2} b$ respectively, in $k_{v}$ momentum space is:

$$
\Phi_{q_{1}}\left(k_{v}^{1}, u, x^{i}\right) * \Phi_{q_{2}}\left(k_{v}^{2}, u, x^{i}\right)=e^{i b\left(n_{2} k_{v}^{1}-n_{1} k_{v}^{2}\right)} \Phi_{q_{1}}\left(k_{v}^{1}, u, x^{i}\right) \Phi_{q_{2}}\left(k_{v}^{2}, u, x^{i}\right),
$$

with the dipole dependence contained in the phase factor, which is invariant under Schrödinger transformations.

Given that the series of deformations considered here is expected to resum into a null dipole theory by the analogue of the Seiberg-Witten map [32], one might ask what this implies for the renormalizability and unitarity of the theory at finite $b$. This question has not been explored in previous literature, although it has been argued that analogous theories with light-like noncommutativity are well-defined, despite the non-locality in one null direction [34]. The reason is that lightcone quantization in which the other (local) null coordinate is treated as the time coordinate results in a lightcone Hamiltonian which is Hermitian. Naively at least the same argument would apply also to null dipole theories, since they are local in the $\left(u, x^{i}\right)$ coordinates.

\subsection{Stress energy tensor}

Next we turn to the stress energy of the deformed theory. It is useful to start the discussion by considering again a simple explicit model which is Schrödinger invariant:

$$
S=\int d^{2} x\left(\partial_{u} \Phi \partial_{v} \Phi+b \partial_{u} \Phi\left(\partial_{v} \Phi\right)^{2}\right) .
$$

This model exhibits the Schrödinger group of symmetries (1.2) presented in the introduction. Let us now consider the Noether current $\mathcal{J}^{i}$ for each symmetry $Q$. For $H$ the corresponding symmetry current is:

$$
\mathcal{H}^{u}=0 ; \quad \mathcal{H}^{v}=\left(\partial_{u} \Phi\right)^{2}\left(1+2 b \partial_{v} \Phi\right) .
$$

For $M$ the symmetry current is:

$$
\mathcal{M}^{u}=\left(\partial_{v} \Phi\right)^{2}\left(1+b\left(\partial_{v} \Phi\right)\right) ; \quad \mathcal{M}^{v}=b \partial_{u} \Phi\left(\partial_{v} \Phi\right)^{2} .
$$

The dilatation current is:

$$
\mathcal{D}^{u}=0 ; \quad \mathcal{D}^{v}=u\left(\partial_{u} \Phi\right)^{2}\left(1+2 b \partial_{v} \Phi\right)
$$


whilst the special conformal current is similar:

$$
\mathcal{C}^{u}=0 ; \quad \mathcal{C}^{v}=u^{2}\left(\partial_{u} \Phi\right)^{2}\left(1+2 b \partial_{v} \Phi\right)
$$

The currents are conserved onshell because of the field equation:

$$
\partial_{u} \partial_{v} \Phi+b \partial_{u} \Phi \partial_{v}^{2} \Phi+2 b \partial_{u} \partial_{v} \Phi \partial_{v} \Phi=0 .
$$

Usually the currents associated with (conformal) isometries can be expressed in terms of a symmetric conserved (traceless) stress energy tensor $T_{i j}$ as

$$
\mathcal{J}^{i}=T^{i j} \zeta_{j}^{Q}
$$

where $\zeta_{j}^{Q}$ is the vector field generating each symmetry, i.e. $\delta_{Q} x^{i}=-\zeta^{Q i}$.

In the case at hand the symmetry currents can all be written as $\mathcal{J}^{i}=t^{i j} \zeta_{j}^{Q}$ where the tensor $t_{i j}$ is:

$$
\begin{array}{ll}
t_{u u}=\left(\partial_{u} \Phi\right)^{2}\left(1+2 b \partial_{v} \Phi\right) ; & t_{v u}=0 ; \\
t_{u v}=b \partial_{u} \Phi\left(\partial_{v} \Phi\right)^{2} ; & t_{v v}=\left(\partial_{v} \Phi\right)^{2}\left(1+b\left(\partial_{v} \Phi\right)\right) .
\end{array}
$$

Under the dilatation symmetry $u \rightarrow \lambda^{2} u$ the tensor $t_{i j}$ scales as:

$$
t_{u u} \rightarrow \lambda^{-4} t_{u u} ; \quad t_{u v} \rightarrow \lambda^{-2} t_{u v} ; \quad t_{v v} \rightarrow \lambda^{0} t_{v v} .
$$

This tensor is conserved but it is not symmetric and it is not traceless. The latter implies that the theory is invariant only under chiral dilatation invariance, rather than the corresponding anti-chiral dilatation invariance. In a relativistic theory one can always find an improvement term $\theta_{i j}=\partial^{l} Y_{l i j}$, where $Y_{l i j}$ is antisymmetric in its first two indices, such that $T_{i j}=t_{i j}+\theta_{i j}$ is symmetric. In the example at hand one can easily prove that such improvement tensor does not exist. ${ }^{5}$ This illustrates a general result:

Any theory in Minkowski spacetime that possesses a conserved, symmetric stress energy tensor $T_{i j}$ is Lorentz invariant.

This can be shown as follows. Minkowski space has an isometry corresponding to Lorentz transformations with Killing vector $\xi^{i}=\omega_{i j} x^{j}$ and $\omega_{i j}$ antisymmetric. Using $T_{i j}$ one can construct the corresponding conserved Lorentz current,

$$
J_{L}^{i}=T^{i j} \omega_{j k} x^{k}
$$

This is conserved because

$$
\partial_{i} J_{L}^{i}=\left(\partial_{i} T^{i j}\right) \omega_{j k} x^{k}+T^{i j} \omega_{i j}=0
$$

For the Schrödinger theory, invariance under translations implies the existence of a conserved stress energy tensor. However, this must be non-symmetric because otherwise the theory would be Lorentz invariant.

\footnotetext{
${ }^{5}$ Assuming $Y$ to exist one obtains from $T^{u v}=T^{v u}$ and (4.95) that $b \partial_{u} \Phi\left(\partial_{v} \Phi\right)^{2}=\partial_{v} Y^{v u v}-\partial_{u} Y^{u v u}$ should hold. This equation, however, does not hold as the deformation is not a total derivative.
} 
When one couples a theory which is Lorentz invariant to background gravity the symmetric and conserved stress energy tensor arises from the variation of the action with respect to the metric. In a non-Lorentz invariant theory one can couple the model to background gravity using vielbeins. The coupling to gravity amounts to replacing curved indices by flat target indices using vielbeins, i.e. $A_{i}$ becomes $e_{i}^{m} A_{m}$, where $m$ is a curved space index. The fact that the underlying theory is not Lorentz invariant means that some of the tangent space indices are open, i.e. not contracted. In this case the non-symmetric energy momentum tensor will naturally emerge from the variation of the action with respect to the vielbein.

For the toy model given above coupling to the vielbein gives

$$
S=\int d^{2} x e\left(e^{m i} e_{i}^{n} \partial_{m} \Phi \partial_{n} \Phi+b e_{\hat{u}}^{m} e_{\hat{v}}^{n} e_{\hat{v}}^{p}\left(\partial_{m} \Phi\right)\left(\partial_{n} \Phi\right)\left(\partial_{p} \Phi\right)\right) \equiv \int d^{2} x e \mathcal{L},
$$

where $e$ is the determinant of the vielbein and the tangent space metric is $g_{i j}=\eta_{i j}$ such that $\eta_{\hat{u} \hat{v}}=1$. For clarity the tangent space indices are denoted $(\hat{u}, \hat{v})$. Defining:

$$
t_{m n}=\frac{e_{n}^{j}}{e} \frac{\delta \mathcal{L}}{\delta e^{m j}}
$$

gives:

$$
t_{m n}=\partial_{m} \Phi \partial_{n} \Phi-g_{m n} \mathcal{L}+b\left(e_{\hat{u} n} e_{\bar{v}}^{p} e_{\hat{v}}^{q}+2 e_{\hat{v} n} e_{\hat{u}}^{p} e_{\hat{v}}^{q}\right) \partial_{m} \Phi \partial_{p} \Phi \partial_{q} \Phi
$$

which in a flat background in which $g_{m n}=\eta_{m n}$ reproduces the tensor $t_{i j}$ above. The simplest way to show that $t_{m n}$ is conserved is the following. Pulling $t_{m n}$ back into tangent space reproduces $t_{i j}$; the latter is manifestly conserved using the field equation:

$$
\square \Phi+D_{\hat{u}}\left(b\left(\partial_{\hat{v}} \Phi\right)^{2}\right)+2 D_{\hat{v}}\left(b \partial_{\hat{u}} \Phi \partial_{\hat{v}} \Phi\right)=0 .
$$

Since

$$
D^{m} t_{m n}=D^{m}\left(e_{m}^{i} e_{n}^{j} t_{i j}\right)=e_{n}^{j} D^{i} t_{i j},
$$

conservation of the pulled back tensor $t_{i j}$ implies conservation of $t_{m n}$.

To summarize, there are two related stress energy operators in the deformed field theory. The first is the symmetric tensor $T_{i j}$ which couples to the metric, but is not conserved in the deformed theory at finite $b$. The second is the operator $t_{m n}$ that couples to the vielbein, which is not symmetric but is conserved. It is the latter operator which is natural in the deformed theory, and thus in the holographic analysis one should trade metric fluctuations for vielbein fluctuations. In other words, one should specify boundary conditions for the vielbein, rather than the metric; this point was noted in [18].

It is interesting to connect this discussion with the conventional description of nonrelativistic theories. Suppose one formulates a Schrödinger invariant theory in $(d-1)$ dimensions, with background coordinates $\left(u, x^{i}\right)$. Then, the natural operators are the energy current $\mathcal{E}$ with components $\left(\mathcal{E}_{u}, \mathcal{E}_{i}\right)$; the mass current $\rho$ with components $\left(\rho_{u}, \rho_{i}\right)$ and the (symmetric) stress tensor $\pi_{i j}$. In the $d$-dimensional realization of the Schrödinger symmetry discussed here, the operator $t_{m n}$ should include these operators. It will however also include additional operators associated with the extra lightcone dimension, which 
are not usually discussed in the lower dimensional realizations. These extra operators are necessarily present because the Schrödinger theory is obtained via a deformation of a relativistic theory in $d$ dimensions.

Before leaving the field theory discussion, it is also useful to summarize which operators are expected to be marginal, irrelevant and relevant in the deformed Schrödinger invariant theory. In the cases of both TMG and the massive vector, the operator which deformed the theory to Schrödinger is irrelevant at finite $k_{v}$; its scaling dimension in both cases is given holographically (for $d=2$ ) as:

$$
\Delta_{s}=1+\sqrt{1+b^{2} k_{v}^{2}}
$$

In the massive vector case the dimension of the other vector operator $X_{u}$ is such that it is also irrelevant for non-zero $k_{v}$. Therefore these operators are all irrelevant in the Schrödinger theory, except for the deforming operator itself with $k_{v}=0$.

Now consider the stress energy tensor $T_{i j}$, at $b=0$. The non-relativistic scaling dimensions of its components are:

$$
\Delta_{s}\left(T_{u u}\right)=4 ; \quad \Delta_{s}\left(T_{u v}\right)=2 ; \quad \Delta_{s}\left(T_{v v}\right)=0,
$$

and thus the components are irrelevant, marginal and relevant, respectively. Away from $b=0$ this is not the conserved operator, but the components of $t_{i j}$ have the same scaling dimensions, see (4.96), and these are protected. Thus from the perspective of the Schrödinger symmetry group, the operator $T_{u u}$ is irrelevant.

Since the operators $X_{v v}\left(k_{v}\right), X_{v}\left(k_{v}\right), X_{u}\left(k_{v}\right)$ and $T_{u u}$ are irrelevant, switching on finite sources for these operators in the deformed theory would be expected to change the UV structure of the theory. Correspondingly, in the holographic dual, finite sources for these operators would be expected to change the asymptotic structure of the dual spacetime. In the subsequent sections we will consider solutions of the bulk linearized equations, and we will indeed see the bulk fields dual to these operators blow up faster at the boundary than the background, demonstrating the fact that these modes change the asymptotic structure of the spacetime.

\section{TMG and the null warped background}

In this section we consider TMG and the null warped $A d S_{3}$ background in more detail. The results presented below will provide a basis for the holographic computation of correlation functions in the next sections. In subsection 5.1 we investigate the variational principle for TMG for finite values of the cutoff. In subsection 5.2 we consider the dilatation operator for linearized fluctuations around the null warped background.

\subsection{The variational principle for TMG}

In this subsection we analyze the variational principle for TMG for finite values of the cutoff. Recall that for ordinary Einstein gravity such an analysis results in the addition of the Gibbons-Hawking term to the Einstein-Hilbert action. For TMG the corresponding 
variational principle is worked out in detail in this section. Note that the results in this section are completely general and independent of the choice of background metric. They can therefore also be used to obtain, for example, the correct on-shell action for black hole solutions in TMG.

We begin by decomposing the background metric in a radial ADM form:

$$
d s^{2}=N^{2} d r^{2}+\gamma_{i j}\left(d x^{i}+N^{i} d r\right)\left(d x^{j}+N^{j} d r\right) .
$$

The connection coefficients are then given by:

$$
\begin{aligned}
\Gamma_{r r}^{r} & =\frac{\dot{N}}{N}+N^{i} B_{i} & \Gamma_{r r}^{i} & =\gamma^{i k} \dot{N}_{i}-N^{i} \Gamma_{r r}^{r}-N^{2} B^{i}-F^{i}{ }_{j} N^{j} \\
\Gamma_{j r}^{i} & =F_{j}{ }^{i}-N^{i} B_{j} & \Gamma_{i j}^{r} & =\frac{1}{N} K_{i j} \\
\Gamma_{r i}^{r} & =B_{i} & \Gamma_{j k}^{i} & =\Gamma_{j k}^{i}[\gamma]-\frac{1}{N} N^{i} K_{j k}
\end{aligned}
$$

where we defined:

$$
F_{i j}=\nabla_{i} N_{j}-N K_{i j}, \quad B_{i}=\frac{1}{N}\left(\partial_{i} N+K_{i j} N^{j}\right) .
$$

A dot denotes a radial derivative. Indices are raised with $\gamma^{i j}$ and $\epsilon_{i j}$ and covariant derivatives $\nabla_{k}$ are defined using $\gamma_{i j}$ as well. Henceforth all $\Gamma_{j k}^{i}$ are those associated to $\gamma_{i j}$. In our conventions the extrinsic curvature is given by:

$$
K_{i j}=\frac{1}{2 N}\left(\nabla_{i} N_{j}+\nabla_{j} N_{i}-\partial_{r} \gamma_{i j}\right)
$$

We henceforth suppose that $N>0$. The Einstein-Hilbert action plus Gibbons-Hawking term then becomes:

$$
S_{\mathrm{EH}}=\frac{1}{16 \pi G_{N}} \int d^{3} x \sqrt{-\gamma} N\left(R[\gamma]-2 \Lambda+K^{2}-K_{i j} K^{i j}\right) .
$$

The Chern-Simons action becomes:

$$
\begin{aligned}
S_{\mathrm{CS}}=\frac{1}{16 \mu \pi G_{N}} \int d^{3} x & \sqrt{-\gamma} \epsilon^{i j}\left(K_{i}^{k} \dot{K}_{k j}+N\left[2 \nabla_{k} \nabla_{i} K_{j}^{k}\right]\right. \\
& \left.+N^{l}\left[\nabla_{k}\left(K_{i}^{k} K_{j l}\right)-2 K_{k l} \nabla_{i} K_{j}^{k}+\frac{1}{2} \epsilon_{l}^{k} \partial_{k} R\right]-\frac{1}{2} \Gamma_{i l}^{k} \partial_{r} \Gamma_{j k}^{l}\right)
\end{aligned}
$$

plus a radial boundary term of the form $\int d^{2} x \sqrt{-\gamma} \epsilon^{i j} K_{i k}\left(\partial_{j} N^{k}\right) /(2 N)$ which vanishes once we gauge-fix $N^{k}=0$ below. Here spatial total derivatives have been omitted. This ADM form of the Chern-Simons action was also found in [48-50].

The Chern-Simons action can be manipulated as follows. First of all, we define $A^{k l}$ via:

$$
\int d^{3} x \sqrt{-\gamma} \epsilon^{i j} \Gamma_{i l}^{k} \partial_{r} \Gamma_{j k}^{l}=\int d^{3} x \sqrt{-\gamma} A^{k l} \dot{\gamma}_{k l}
$$

up to total spatial derivatives. Furthermore, the first term in (5.6) can be rewritten. To this end we decompose the extrinsic curvature as:

$$
K_{i j}=k_{i j}+\bar{k}_{i j}+\frac{1}{2} k \gamma_{i j}
$$


with:

$$
\begin{aligned}
k & =\gamma^{i j} K_{i j} \\
k_{i j} & =P_{i}{ }^{k}\left(K_{k j}-\frac{1}{2} k \gamma_{k j}\right)=\frac{1}{2}\left(\delta_{i}^{k}+\epsilon_{i}^{k}\right)\left(K_{k j}-\frac{1}{2} k \gamma_{k j}\right) ; \\
\bar{k}_{i j} & =\bar{P}_{i}{ }^{k}\left(K_{k j}-\frac{1}{2} k \gamma_{k j}\right)=\frac{1}{2}\left(\delta_{i}^{k}-\epsilon_{i}^{k}\right)\left(K_{k j}-\frac{1}{2} k \gamma_{k j}\right) .
\end{aligned}
$$

Notice that $k_{i j}$ and $\bar{k}_{i j}$ are symmetric, traceless and chiral (or antichiral), so:

$$
\begin{aligned}
& \gamma^{i j} k_{i j}=0, \quad k_{i j}=k_{j i}, \quad \bar{P}_{i}^{k} k_{k j}=0 ; \\
& \gamma^{i j} \bar{k}_{i j}=0, \quad \bar{k}_{i j}=\bar{k}_{j i}, \quad P_{i}{ }^{k} \bar{k}_{k j}=0 .
\end{aligned}
$$

These equations imply that $k_{i j}$ and $\bar{k}_{i j}$ each have a single independent component. Using (5.8) we may rewrite:

$$
\begin{aligned}
\int d^{3} x \sqrt{-\gamma} \epsilon^{i j} K_{i}^{k} \dot{K}_{k j}= & \int d^{3} x \sqrt{-\gamma}\left(-2 k_{k}^{j} \partial_{r} \bar{k}_{j}^{k}-N^{l} \nabla_{l}\left(k_{k}^{j} \bar{k}_{j}^{k}\right)+N K k_{k}^{j} \bar{k}_{j}^{k}\right) \\
& +\int d^{2} x \sqrt{-\gamma} k_{k}^{j} \bar{k}_{j}^{k} .
\end{aligned}
$$

The last boundary term should be canceled by a boundary term in the action.

We now regard $k_{i}^{j}, \bar{k}_{i}^{j}$ and $k$ as independent variables, together with $\gamma_{i j}, N^{i}$ and $N$. On the other hand, $K_{i j}$ should henceforth be understood as in (5.8). We need to introduce a single Lagrange multiplier $\Pi^{i j}$ enforcing (5.4):

$$
\int d^{3} x \sqrt{-\gamma} \Pi^{i j}\left(\dot{\gamma}_{i j}+2 N K_{i j}-\nabla_{i} N_{j}-\nabla_{j} N_{i}\right) .
$$

The combined action $S$ consists of the Einstein-Hilbert term, the Chern-Simons term, the constraint (5.12) and two boundary terms: the Gibbons-Hawking term and a new term that cancels the last term in (5.11). The total action then becomes:

$$
S=\frac{1}{16 \pi G_{N}} \int d^{3} x \sqrt{-\gamma}\left(-\frac{2}{\mu} k_{k}^{j} \partial_{r} \bar{k}_{j}^{k}+\left(\Pi^{j k}-\frac{1}{2 \mu} A^{j k}\right) \dot{\gamma}_{j k}+N \mathcal{H}+N^{l} \mathcal{P}_{l}\right)
$$

with:

$$
\begin{aligned}
& \mathcal{H}=R[\gamma]-2 \Lambda+\frac{1}{2} k^{2}-2 k_{k}^{j} \bar{k}_{j}^{k}+2 \Pi_{j}^{i}\left(k_{i}^{j}+\bar{k}_{i}^{j}\right)+\Pi_{k}^{k} k+\frac{2}{\mu} \nabla^{k} \nabla_{i}\left(k_{k}^{i}-\bar{k}_{k}^{i}\right)+\frac{1}{\mu} k k_{k}^{j} \bar{k}_{j}^{k} \\
& \mathcal{P}_{l}=2 \nabla^{i} \Pi_{i l}+\frac{1}{\mu}\left[\nabla_{l}\left(k_{k}^{j} \bar{k}_{j}^{k}\right)+\epsilon^{i j} \nabla_{k}\left(K_{i}^{k} K_{j l}\right)-2 \epsilon^{i j} K_{k l} \nabla_{i} K_{j}^{k}+\frac{1}{2} \epsilon_{l}^{k} \partial_{k} R\right] .
\end{aligned}
$$

The variation of the action is given by:

$$
\delta S=\frac{1}{16 \pi G_{N}}\left[\int d^{3} x(\mathrm{eom})+\int d^{2} x \sqrt{-\gamma}\left(-\frac{2}{\mu} k_{k}^{j} \delta \bar{k}_{j}^{k}+\left(\Pi^{j k}-\frac{1}{2 \mu} A^{j k}\right) \delta \gamma_{j k}\right)\right]
$$

so we find a well-defined variational principle if we define $\gamma_{i j}$ and $\bar{k}_{i}^{j}$ as the boundary data. Notice that we may also lower the index on $\bar{k}_{i}^{j}$ since using $\bar{k}_{i j}=\bar{k}_{i}^{k} \gamma_{k j}$ rather than $\bar{k}_{i}^{j}$ is a simple change of variables that does not affect the variational principle. 
Below we will need to evaluate the first variation of the on-shell action and we therefore need the on-shell expression for $\Pi_{i j}$. This can be obtained by varying the action (5.13) with respect to $k_{i j}, \bar{k}_{i j}$ and $k$. In the gauge where $N^{i}=0$ and $N=N(r)$ (which we use below) we obtain:

$$
\Pi_{i j}=\frac{1}{\mu N} \partial_{r}\left(\bar{k}_{i j}-k_{i j}\right)+k_{i j}+\bar{k}_{i j}-\gamma_{i j}\left(\frac{1}{2} k+\frac{1}{2 \mu} k_{l}^{m} \bar{k}_{m}^{l}\right)+\frac{1}{2 \mu} k\left(\bar{k}_{i j}-k_{i j}\right) .
$$

The other equations of motion combine to (2.4) and (5.9) with (5.4), as expected.

\subsection{The dilatation operator in the null warped background}

A convenient way to obtain the correct counterterms in the usual AlAdS backgrounds is the radial Hamiltonian method $[37,38]$. In this method one expands the conjugate momenta to the bulk fields in terms of eigenfunctions of a covariant dilatation operator, denoted $\delta_{\mathcal{D}}$, which is asymptotically equal to the radial derivative.

As an example, consider a $(d+1)$-dimensional asymptotically locally AdS background of the form:

$$
d s^{2}=d \bar{r}^{2}+\gamma_{i j} d x^{i} d x^{j} \quad \gamma_{i j}=e^{2 \bar{r}} g_{(0) i j}+\ldots
$$

where $\bar{r}$ is a new radial coordinate relative to $(3.1): r=e^{-\bar{r}}$ so the boundary is now at $\bar{r} \rightarrow \infty$. To keep the notation uncluttered in this and the following section the coordinate $\bar{r}$ will henceforth be denoted as $r$. In anticipation of what follows we also consider a massive symmetric transverse traceless second rank tensor, $\Phi_{\nu}^{\mu}$, satisfying

$$
\left(\nabla_{\mu} \nabla^{\mu}+\left(2-m^{2}\right)\right) \Phi_{\nu}^{\mu}=0, \quad \Phi_{\mu}^{\mu}=0 \quad \nabla_{\mu} \Phi_{\nu}^{\mu}=0,
$$

where $m^{2}=\Delta(\Delta-d)$. This field is dual to an operator of dimension $\Delta$. In a spacetime of the form (5.17) the symmetric tensor has a radial expansion which to leading order has the form:

$$
\Phi_{j}^{i}\left(r, x^{i}\right)=e^{(\Delta-d) r} \phi_{(0) j}^{i}+\ldots
$$

Consider now an object like the regulated on-shell action $S$ for this configuration. It is a functional of the boundary data for the fields at the cutoff surface, so we may write:

$$
S\left[\gamma_{i j}, \Phi_{j}^{i}\right]
$$

where it is understood that $\gamma_{i j}$ and $\Phi_{j}^{i}$ are the induced fields on the cutoff surface. Since the action does not depend explicitly on the radial coordinate $r$, its radial dependence is inherited completely from the radial dependence of $\gamma_{i j}$ and $\Phi_{j}^{i}$. For its radial derivative we may therefore write:

$$
\dot{S}=\int d^{d} x \dot{\gamma}_{i j} \frac{\delta S}{\delta \gamma_{i j}}+\int d^{d} x \dot{\Phi}_{j}^{i} \frac{\delta S}{\delta \Phi_{j}^{i}}
$$

where a dot denotes a radial derivative. Asymptotically we may use (5.17) and (5.19) to find that:

$$
\partial_{r} \sim \int d^{d} x 2 \gamma_{i j} \frac{\delta}{\delta \gamma_{i j}}+\int d^{d} x(\Delta-d) \Phi_{j}^{i} \frac{\delta}{\delta \Phi_{j}^{i}}=\delta_{\mathcal{D}}
$$


at least when acting on a functional like the on-shell action. The tilde symbolizes equality up to terms that vanish as $r \rightarrow \infty$. The functional operator $\delta_{\mathcal{D}}$ is precisely the field theory dilatation operator and this relation between the radial derivative and dilatation operator reflects the fact that the RG scale becomes geometric in gauge/gravity duality. This relation is useful for the renormalization of the action in the following way. Normally the divergences in the bare on-shell action are organized in terms of degree of divergence, i.e. in terms of a power series in $e^{r}$. However we may also organize these terms in terms of eigenfunctions of the dilatation operator $\delta_{\mathcal{D}}$. Just as in the ordinary method the equations of motion are then used to determine the exact form of this expansion. The advantage of expanding in eigenfunctions of $\delta_{\mathcal{D}}$ is that $\delta_{\mathcal{D}}$ is fully covariant and as a consequence the divergences will be organized directly in a covariant expansion as well. This greatly simplifies the analysis of the counterterms which one can essentially pick to be (minus) the divergent components in this expansion. We will see an example worked out below. (In the presence of a conformal anomaly this story is altered somewhat as we will also demonstrate below.)

Let us now formulate a similar operator for the null warped background. For concreteness we are working in TMG; the analysis for the massive vector model is completely analogous.

For TMG the on-shell action $S$ depends not only on the induced metric $\gamma_{i j}$ but also on a component of the extrinsic curvature $\bar{k}_{j}^{i}$. For the on-shell action we therefore write this time:

$$
S\left[\gamma_{i j}, \bar{k}_{i}^{j}\right] .
$$

For the radial derivative on such a functional we find:

$$
\partial_{r}=\int d^{2} x \dot{\gamma}_{i j} \frac{\delta}{\delta \gamma_{i j}}+\int d^{2} x \dot{\bar{k}}_{i}^{j} \frac{\delta}{\delta \bar{k}_{i}^{j}}
$$

Now, for the AlAdS spacetimes we substituted at this point the general leading-order radial behavior of the fields (given in (5.17) and (5.19)) to obtain a covariant dilatation operator which was valid for all AlAdS spacetimes. On the other hand, for the null warped case we do not have a precise definition of an 'asymptotically null warped spacetime' and we do not know what the leading-order behavior of the fields for the general solution should be. Therefore we will from now on narrow down our analysis to a specific class of solutions. Specifically, the analysis below is valid for solutions that asymptote to the null warped AdS solution (2.5). The null warped background metric can be written as:

$$
d s^{2}=d r^{2}+\gamma_{i j} d x^{i} d x^{j} \quad \gamma_{i j}=\zeta_{i j}+b_{i j}
$$

where we introduced

$$
\zeta_{i j} d x^{i} d x^{j}=e^{2 r} 2 d u d v \quad b_{i j} d x^{i} d x^{j}=-e^{4 r} b^{2} d u^{2}
$$

Notice that $\zeta_{i j}$ is simply $e^{2 r} \eta_{i j}$ and in particular is not equal to the induced metric $\gamma_{i j}$ on slices of constant $r$. On this background we find that

$$
K_{i j}=\frac{1}{2} \dot{\gamma}_{i j}=\gamma_{i j}+b_{i j}
$$


from which it is easy to find that $\bar{k}_{i j}=b_{i j}$ (in conventions where $\sqrt{-\gamma} \epsilon^{u v}=+1$ ) and therefore:

$$
\bar{k}_{i}^{j}=\gamma^{j k} b_{k i}=-e^{2 r} b^{2} \delta_{i}^{u} \delta_{v}^{j} .
$$

Note that $\bar{k}_{i}^{j}$ is a second rank symmetric transverse traceless tensor that satisfies (5.18) for $d=2, \Delta=4$ in an $A d S_{3}$ background.

Let us now go back to (5.24). Using the above asymptotics we obtain

$$
\partial_{r} \sim \int d^{2} x\left(2 \gamma_{i j}+2 \bar{k}_{i j}\right) \frac{\delta}{\delta \gamma_{i j}}+\int d^{2} x 2 \bar{k}_{i}^{j} \frac{\delta}{\delta \bar{k}_{i}^{j}}
$$

Let us further change variables from $\left(\gamma_{i j}, \bar{k}_{i}^{j}\right)$ to $\left(\zeta_{i j}, \bar{k}_{i}^{j}\right)$. This yields

$$
\partial_{r} \sim \int d^{2} x\left(2 \zeta_{i j} \frac{\delta}{\delta \zeta_{i j}}+2 \bar{k}_{i}^{j} \frac{\delta}{\delta \bar{k}_{i}^{j}}\right)=\delta_{\mathcal{D}}
$$

which is precisely the dilatation operator that corresponds to a deformation of the CFT (on $2 \mathrm{~d}$ Minkowski spacetime) by a dimension 4 operator, cf (5.22).

The most useful representation of the radial derivative however is obtained by changing variables from $\left(\zeta_{i j}, \bar{k}_{i}^{j}\right)$ to $\left(\zeta_{i j}, b^{i j}=\zeta^{i k} \bar{k}_{k}^{j}\right)$. This results in

$$
\partial_{r} \sim \int d^{2} x 2 \zeta_{i j} \frac{\delta}{\delta \zeta_{i j}}=\delta_{\mathcal{D}}
$$

Note that $b^{v v}=-b^{2}$ and $b^{u u}=b^{u v}=0$, so after all these change of variables

$$
S\left[\gamma_{i j}, \bar{k}_{j}^{i}\right] \rightarrow S\left[\zeta_{i j}, b^{2}\right]
$$

and in this functional the radial derivative is represented by (5.31). We will use this form of the operator $\delta_{\mathcal{D}}$ to organize the divergences in the next section.

It is also interesting to use this discussion to understand how to couple a metric to the dual deformed theory. Recalling that the deformation is by a $(3,1)$ operator, a simple toy model that captures this behavior is the scalar field theory used in section 4 :

$$
S=\int d^{2} x\left(\partial_{u} \Phi \partial_{v} \Phi+b \partial_{u} \Phi\left(\partial_{v} \Phi\right)^{3}\right) .
$$

For this model the coupling to the metric is the following

$$
S[\zeta, \bar{k}]=\int d^{2} x \sqrt{-\zeta}\left[\zeta^{i j} \partial_{i} \Phi \partial_{j} \Phi+\bar{k}_{j}^{i}\left(\partial_{i} \Phi \partial_{k} \Phi \partial_{l} \Phi \partial_{m} \Phi \zeta^{j k} \zeta^{l m}\right)\right]
$$

Under Weyl transformations $\left(\partial_{i} \Phi \partial_{k} \Phi \partial_{l} \Phi \partial_{m} \Phi \zeta^{j k} \zeta^{l m}\right)$ transforms as a dimension 4 operator. Then

$$
T_{i j}=\frac{2}{\sqrt{-\zeta}} \frac{\delta S}{\delta \zeta^{i j}} .
$$

Note that this operator is not conserved except when $b=0$. 


\section{Scalar field in the Schrödinger background}

In section 4 we discussed the effect of irrelevant deformations preserving Schrödinger symmetry from the field theory perspective. In this section we holographically compute the two-point function of a scalar operator to illustrate the consequences of the finite irrelevant deformation; this computation displays the same structure as found in the field theory. For computational simplicity, we work in $d=2$, so three bulk dimensions, but the generalization to higher dimensions would be straightforward.

\subsection{Action and equations of motion}

For a massive scalar field the action is:

$$
S=-\frac{1}{2} \int d^{3} x \sqrt{-G}\left(\partial_{\mu} \Phi \partial^{\mu} \Phi+m^{2} \Phi^{2}\right) .
$$

The equation of motion in the metric (5.25) becomes:

$$
\ddot{\Phi}+2 \dot{\Phi}+\square_{\zeta} \Phi-\left(m^{2}-b^{2} \partial_{v}^{2}\right) \Phi=0
$$

where a dot denotes a radial derivative and $\square_{\zeta}=2 e^{-2 r} \partial_{u} \partial_{v}$. This equation of motion is precisely the equation of motion for a massive scalar in AdS:

$$
\ddot{\Phi}+2 \dot{\Phi}+\square_{\zeta} \Phi-M^{2} \Phi=0
$$

with the effective mass squared $M^{2}=m^{2}-b^{2} \partial_{v}^{2}$ which depends on the lightcone momentum. ${ }^{6}$ If we Fourier transform $\tilde{\Phi}\left(k_{u}, k_{v}\right)=\int d u d v \exp \left(i k_{u} u+i k_{v} v\right) \Phi(u, v)$ then the asymptotic solution to this equation is of the form:

$$
\Phi\left(r, k_{u}, k_{v}\right)=e^{\left(\Delta_{s}-2\right) r}\left(\phi_{(0)}(k)+\ldots+e^{-\left(2 \Delta_{s}-2\right) r} \phi_{\left(2 \Delta_{s}-2\right)}(k)+\ldots\right)
$$

where here and below we drop the tilde from $\tilde{\Phi}$ and $\Delta_{s}=1+\sqrt{1+m^{2}+b^{2} k_{v}^{2}}$. For $b^{2}=0$ one would find that $\Delta_{s}=\Delta$ with $\Delta=1+\sqrt{1+m^{2}}$ the scaling dimension of the dual operator. For nonzero $b^{2}$ we shall see how $\Delta_{s}$ will be the scaling dimension under the dilatation operator $\mathcal{D}$ that appears in the Schrödinger group (1.2); the fact that the scaling dimension depends on the lightcone momentum $k_{v}$ was noted previously in $[1,2,15]$. The generalization to arbitrary dimensions is:

$$
\Delta_{s}=\frac{d}{2}+\sqrt{\frac{d^{2}}{4}+m^{2}+b^{2} k_{v}^{2}}
$$

\footnotetext{
${ }^{6}$ In this paper we suppress real-time issues. In [51] it was shown that the Scrödinger spacetimes do not admit a global time function and it was argued that the initial value problem for (6.2) is not well-defined, unless one removes the $k_{v}=0$ modes. In the context of AdS/CFT the proper set up to investigate the initial value problem is the framework of $[52,53]$. This framework was adapted to the Schrödinger case in [54], but the analysis for $k_{v}=0$ is not given there. This is an interesting issue that we leave for future work.
} 
Notice that $\Delta_{s}$ explicitly depends on the lightcone momentum $k_{v}$. The expression (6.4) is valid for generic values of $\Delta_{s}$; exceptions occur when $\Delta_{s} \in\{1,2,3, \ldots\}$ and we discuss these separately below.

At this point it is important to make a distinction between $b^{2}$ positive and negative. For $b^{2}$ negative (which is allowed in TMG but not in the massive vector model), there is a critical value of the lightcone momentum $k_{v}^{2}=m^{2} /\left|b^{2}\right|$ above which the effective mass squared drops below the BF bound and the corresponding operator dimension becomes complex. For $b^{2}$ positive the effective mass squared is always positive, and the operator dimension grows linearly with large momentum $k_{v}$.

We now proceed to analyze the massive scalar using Hamiltonian methods of holographic renormalization. At a technical level the similarity of (6.2) with the equation of motion in pure AdS means that the analysis will proceed along the same lines as for pure AdS, but with an important conceptual difference: the counterterms depend explicitly on $\Delta_{s}$ and therefore on the lightcone momentum $k_{v}$ in such a way that they are non-local in the $v$ direction.

\subsection{The dilatation operator and asymptotic expansions}

The on-shell regularized action $S$ for $\Phi$ is a functional of $\gamma_{i j}, \bar{k}_{i}^{j}$ and $\Phi$. In this section we treat the scalar in a fixed null warped AdS background, so we may replace $\gamma_{i j}$ and $\bar{k}_{i}^{j}$ with their background values and furthermore perform a change of variables to $\zeta_{i j}$ and $b^{i j}$. We then write:

$$
S\left[\zeta_{i j}, b^{i j}, \Phi\right]
$$

where $b^{v v}=-b^{2}$. Again, all radial dependence of such a functional resides in the radial dependence of its arguments $\zeta_{i j}, b^{i j}$ and $\Phi$. The radial derivative of $S$ can therefore be written as:

$$
\begin{aligned}
\partial_{r} & =\int d^{2} k \dot{\zeta}_{i j}(k) \frac{\delta}{\delta \zeta_{i j}(k)}+\int d^{2} k \dot{b}^{i j}(k) \frac{\delta}{\delta b^{i j}(k)}+\int d^{2} k \dot{\Phi}(k) \frac{\delta}{\delta \Phi(k)} \\
& =\int d^{2} k 2 \zeta_{i j}(k) \frac{\delta}{\delta \zeta_{i j}(k)}+\int d^{2} k \Pi(k) \frac{\delta}{\delta \Phi(k)}
\end{aligned}
$$

where we substituted the explicit expressions for the radial derivatives and defined $\Pi=\dot{\Phi}$. Notice that we are again working in Fourier space. By Hamilton-Jacobi or by explicit computation we obtain that:

$$
\Pi=\frac{1}{\sqrt{-\zeta}} \frac{\delta I}{\delta \Phi}
$$

where $I$ is the onshell action and we used that $\operatorname{det}(G)=\operatorname{det}(\zeta)$. From this expression it follows that $\Pi$, just like $I$, can also be regarded as a functional of $\zeta_{i j}, b^{i j}$ and $\Phi$ and therefore the radial derivative acting on $\Pi$ can also be written in the form (6.7):

$$
\dot{\Pi}=\int d^{2} k 2 \zeta_{i j}(k) \frac{\delta \Pi}{\delta \zeta_{i j}(k)}+\int d^{2} k \Pi(k) \frac{\delta \Pi}{\delta \Phi(k)} .
$$

Having found the asymptotic solution, the next step in the Hamiltonian holographic renormalization is to expand the divergences in eigenfunctions of the dilatation-like operator $\delta_{\mathcal{D}}$ 
defined in (5.31). We write it in Fourier space as:

$$
\delta_{\mathcal{D}}=\int d^{2} k 2 \zeta_{i j}(k) \frac{\delta}{\delta \zeta_{i j}(k)}+\int d^{2} k\left(\Delta_{s}-2\right) \Phi(k) \frac{\delta}{\delta \Phi(k)}
$$

We now want to organize $\Pi$ in terms of eigenfunctions of the dilatation operator $\delta_{\mathcal{D}}$. We therefore write:

$$
\Pi=\Pi_{\left(2-\Delta_{s}\right)}+\Pi_{\left(4-\Delta_{s}\right)}+\Pi_{\left(6-\Delta_{s}\right)}+\ldots+\Pi_{\left(\Delta_{s}\right)}+\ldots
$$

where by definition

$$
\delta_{\mathcal{D}} \Pi_{(s)}=-s \Pi_{(s)} .
$$

From (6.4) we obtain to leading order in the radial expansion that:

$$
\Pi=\left(\Delta_{s}-2\right) \Phi+\ldots
$$

and therefore we immediately find that $\Pi_{\left(2-\Delta_{s}\right)}=\left(\Delta_{s}-2\right) \Phi$ which is in fact how we obtained the leading-order weight $\left(2-\Delta_{s}\right)$ in (6.11). Furthermore, substituting this in (6.7) shows immediately that:

$$
\partial_{r} \sim \delta_{\mathcal{D}}
$$

To find an expression for the subleading terms we rewrite the equation of motion (6.2) as:

$$
\dot{\Pi}+2 \Pi-k_{\zeta}^{2} \Phi-\left(m^{2}-b^{2} k_{v}^{2}\right) \Phi=0
$$

where $k_{\zeta}^{2} \equiv \zeta^{i j} k_{i} k_{j}=2 e^{-2 r} k_{u} k_{v}$. We then replace $\dot{\Pi}$ with (6.9), substitute (6.11) and use (6.12) to collect terms of equal dilatation weight. This results in the following expressions for the subleading terms:

$$
\begin{aligned}
\Pi_{\left(4-\Delta_{s}\right)} & =\frac{1}{2 \Delta_{s}-4} k_{\zeta}^{2} \Phi \\
\Pi_{\left(6-\Delta_{s}\right)} & =\frac{-1}{\left(2 \Delta_{s}-4\right)^{2}\left(2 \Delta_{s}-6\right)} k_{\zeta}^{4} \Phi \\
\Pi_{\left(2 m-\Delta_{s}\right)} & =c\left(m, \Delta_{s}\right) k_{\zeta}^{2 m-2} \Phi
\end{aligned}
$$

where the coefficients $c\left(m, \Delta_{s}\right)$ can be determined recursively. The above expansion continues up to the terms with weight $-\Delta_{s}$. At this order we find that the expression for $\Pi_{\Delta_{s}}$ is undetermined for generic values of $\Delta_{s}$, whereas if $\Delta_{s} \in\{1,2,3, \ldots\}$ we can only satisfy the equation of motion if we include in the expansion an inhomogeneous term of the form:

$$
\Pi=\ldots+r \tilde{\Pi}_{\left(\Delta_{s}\right)}+\ldots
$$

with

$$
\delta_{\mathcal{D}} \tilde{\Pi}_{\left(\Delta_{s}\right)}=-\tilde{\Pi}_{\left(\Delta_{s}\right)}
$$

after which we obtain that:

$$
\delta_{\mathcal{D}} \Pi_{\left(\Delta_{s}\right)}=-\Delta_{s} \Pi_{\left(\Delta_{s}\right)}+\tilde{\Pi}_{\left(\Delta_{s}\right)} .
$$

The equation of motion then fixes:

$$
\tilde{\Pi}_{\left(\Delta_{s}\right)}=\tilde{c}\left(\Delta_{s}\right) k_{\zeta}^{2 \Delta_{s}-2} \Phi
$$

where the prefactor $\tilde{c}\left(\Delta_{S}\right)$ is also determined by the equation of motion. In those cases $\Pi_{\Delta_{s}}$ is still undetermined. 


\subsection{Renormalization of the action}

The bare on-shell action has the form:

$$
S_{\text {bare }}=\frac{1}{2} \int d^{2} k \sqrt{-\zeta} \Phi(-k) \Pi(k)
$$

where $\sqrt{-\zeta}=e^{2 r}$ so in particular it is independent of $k$. Substituting the expansion (6.11), we find divergences that for generic $\Delta_{s}$ can be removed by adding the counterterm action:

$$
S_{\mathrm{ct}}=-\frac{1}{2} \int d^{2} k \sqrt{-\zeta} \Phi(-k) \sum_{\left(2-\Delta_{s}\right) \leq s<\Delta_{s}} \Pi_{(s)}(k)
$$

Notice that for $b^{2}=0$ these counterterms would be local functions of the boundary data $\Phi$, which can be seen directly from the explicit expressions (6.16). This is of course required by the locality and the renormalizability of the dual theory. Transforming back to position space we would then find the standard covariant counterterms. To emphasize this fact we use the 'covariant' notation $\sqrt{-\zeta}$ rather than simply $e^{2 r}$, even when we write the counterterms in Fourier space like in (6.22). For nonzero $b^{2}$ the explicit coefficients involving $\Delta_{s}=1+\sqrt{1+m^{2}+b^{2} k_{v}^{2}}$ make the counterterms nonlocal in the $v$-direction. On the other hand, the counterterms do remain local in the $u$-direction.

We mentioned before that we need an inhomogeneous term in the expansion (6.11) at the integer values of $\Delta_{s}$. To see how this affects the holographic renormalization we consider the particular example where $\Delta_{s} \approx 3$. If $\Delta_{s}$ is slightly less than 3 , we need the counterterms:

$$
S_{\mathrm{ct}, \Delta_{s} \lesssim 3}=-\frac{1}{2} \int d^{2} k \sqrt{-\zeta}\left(\left(\Delta_{s}-2\right) \Phi^{2}+\frac{k_{\zeta}^{2} \Phi^{2}}{2 \Delta_{s}-4}\right)
$$

where $\Phi^{2}=\Phi(-k) \Phi(k)$. At $\Delta_{s}=3$ we need:

$$
S_{\mathrm{ct}, \Delta_{s}=3}=-\frac{1}{2} \int d^{2} k \sqrt{-\zeta}\left(\Phi^{2}+\frac{1}{2} k_{\zeta}^{2} \Phi^{2}-\frac{1}{4} k_{\gamma}^{4} \Phi^{2} r\right)
$$

and when $\Delta_{s}$ is slightly bigger than three we find:

$$
S_{\mathrm{ct}, \Delta_{s} \gtrsim 3}=-\frac{1}{2} \int d^{2} k \sqrt{-\zeta}\left(\left(\Delta_{s}-2\right) \Phi^{2}-\frac{k_{\zeta}^{2} \Phi^{2}}{2 \Delta_{s}-4}-\frac{k_{\zeta}^{4} \Phi^{2}}{\left(2 \Delta_{s}-6\right)\left(2 \Delta_{s}-4\right)^{2}}\right)
$$

Of course, we may include both of the above counterterms involving $k_{\zeta}^{4}$ in the first case as well, as it does not play a role when $\Delta_{s} \lesssim 3$. However, the last counterterm in the case $\Delta_{s} \gtrsim 3$ becomes singular when $\Delta_{s}=3$ and conversely the inhomogeneous counterterm (so the last term in (6.24) is divergent for $\Delta_{s} \gtrsim 3$.

Adding (6.21) and (6.22) we find the renormalized action to be:

$$
S_{\mathrm{ren}}=S_{\mathrm{bare}}+S_{\mathrm{ct}}=\frac{1}{2} \int d^{2} k \sqrt{-\zeta} \Phi(-k) \Pi_{\left(\Delta_{s}\right)}(k)
$$

and the one-point function is then given by:

$$
\langle\mathcal{O}(k)\rangle=\lim _{r \rightarrow \infty} \frac{e^{\Delta_{s} r}}{\sqrt{-\zeta}} \frac{\delta S_{r e n}}{\delta \Phi(k)}=\lim _{r \rightarrow \infty} e^{\Delta_{s} r} \Pi_{\left(\Delta_{s}\right)}(k) .
$$


If $\Delta_{s}(k)$ is non-integral the one point function for the operator $\mathcal{O}(u, k)$ can be explicitly written as:

$$
\langle\mathcal{O}(k)\rangle=-\left(2 \Delta_{s}-2\right) \phi_{\left(2 \Delta_{s}-2\right)}(k) .
$$

The one point function at integral $\Delta_{s}(k)$ receives additional contributions.

To evaluate the two point function, we note that the complete regular solution of the field equations can be expressed in terms of a Bessel function:

$$
\begin{aligned}
\Phi(k, \rho) & =\phi_{(0)}(k) \frac{2^{2-\Delta_{s}}|k|^{\Delta_{s}-1}}{\Gamma\left(\Delta_{s}-1\right)} e^{-r} K_{\Delta_{s}-1}\left(|k| e^{-r}\right) \\
& =\phi_{(0)}(k) e^{\left(\Delta_{s}-2\right) r}\left(1+\ldots+(|k| / 2)^{2 \Delta_{s}-2} \frac{\Gamma\left(1-\Delta_{s}\right)}{\Gamma\left(\Delta_{s}-1\right)} e^{-\left(2 \Delta_{s}-2\right) r}+\ldots\right)
\end{aligned}
$$

where $|k|^{2}=2 k_{u} k_{v}$ and the expressions are again valid for the non-integral values of $\Delta_{s}$. Substituting this regular solution into $(6.28)$ we find that:

$$
\langle\mathcal{O}(k)\rangle=-2\left(\Delta_{s}-1\right)(|k| / 2)^{2 \Delta_{s}-2} \frac{\Gamma\left(1-\Delta_{s}\right)}{\Gamma\left(\Delta_{s}-1\right)} \phi_{(0)}(k),
$$

and thus the two point function is given by:

$$
\langle\mathcal{O}(k) \mathcal{O}(-k)\rangle=-2\left(\Delta_{s}-1\right)(|k| / 2)^{2 \Delta_{s}-2} \frac{\Gamma\left(1-\Delta_{s}\right)}{\Gamma\left(\Delta_{s}-1\right)}
$$

(Note that this expression is not valid at $\Delta_{s} \in\{1,2,3, \ldots\}$ where the radial behavior changes and logarithmic counterterms are required.)

\subsection{Interpretation of the result}

The result (6.31) is of the expected form for a Schrödinger invariant theory: as reviewed in section 4 , the two point function for an operator of dimension $\Delta_{s}$ should be of the form

$$
\left\langle\mathcal{O}_{\Delta_{s}}\left(u, k_{v}\right) \mathcal{O}_{\Delta_{s}}\left(0,-k_{v}\right)\right\rangle=c_{\Delta_{s}, k_{v}} \delta_{\Delta, \Delta_{s}} u^{-\Delta_{s}}
$$

where $c_{\Delta_{s}, k_{v}}$ is the normalization obtained from (6.31). Recalling that the Fourier transform of $u^{-\Delta_{s}}$ is proportional to $k_{u}^{\Delta_{s}-1}$ we see the expected behavior. The normalization of the correlator does not however agree with earlier computations such as [54-56] in which holographic renormalization was not carried out. (The form of the correlators found in these works otherwise agrees with that found here, see also $[57,58]$ for further computations of correlation functions in warped $A d S_{3}$.) Already in an AdS background, i.e. $b=0$, the two point functions have incorrect normalizations if holographic renormalization is not carried out; this incorrect normalization was pointed out in [59] and the correct normalization derived by holographic renormalization is given in [12].

Our computations also agree with the picture given in section 4. In particular, the counterterms are nonlocal but the nonlocality is restricted to the $v$ lightcone direction. Therefore it is meaningful to compute the correlation function as a function of $u$ and the result (6.32) is scheme-independent away from $u=0$. 
Note that an alternative way to derive the counterterms in the case of asymptotically AdS spacetimes is to impose the appropriate variational problem for spacetimes with a (non-degenerate) conformal boundary [60]. As noted earlier, the Schrödinger spacetimes are outside this framework and these results do not carry over automatically. In a recent paper [61] the variational problem was analyzed for spacetimes with general asymptotics and it was found that in general non-local boundary terms may be required. In the context of holography, one must understand the physics behind possible non-local boundary terms, i.e. whether or not such non-local counterterms can be associated with a dual (non-local) quantum field theory.

Let us note in addition that the counterterms are actually of precisely the same form as in AdS, except for the implicit lightcone momentum dependence in the quantity $\Delta_{s}$. Schrödinger symmetry does not enforce this; more general counterterms with arbitrary $k_{v}$ dependence would also respect the required symmetry. It seems possible that this specific form of the counterterms, together with the simple expression for the scaling dimension relative to that in the original CFT, follows from the null dipole picture.

\section{Linearized analysis for TMG}

In this section we consider the linearized TMG bulk equations of motion around the Schrödinger background (1.1) and present the most general solution of the linearized equations. In particular, this solution contains sources for both the dual energy momentum tensor and for the deforming operator $X_{v v}$. The general linearized solution allows us to compute two-point functions in the deformed field theory of both the energy-momentum tensor and the deforming operator $X_{v v}$. To obtain these two-point functions from an on-shell action the usual procedure of holographic renormalization needs to be carried out.

We should emphasize that to carry out holographic renormalization one needs to obtain the most general asymptotic solution of the field equations. In earlier work the most general solutions were not considered, but this corresponds to switching off or constraining sources in the dual field theory. Systematic renormalization requires the complete set of divergences to be isolated, and then these divergences should be canceled by appropriate counterterms. In the case at hand, the counterterm action would be expected to be non-covariant, respecting only the Schrödinger symmetry, and non-local in the lightcone direction.

The section is split into the following parts. We first consider the linearized equations of motion around the null warped background and solve them explicitly. Then we substitute these solutions into the on-shell action, isolate the divergences and use holographic renormalization to render it finite. We may then functionally differentiate this finite on-shell action with respect to the sources to obtain correlation functions.

\subsection{Linearized bulk equations and solutions}

In this section we consider the linearized TMG bulk equations of motion around the background (1.1) and present the general solution. 
Background and equations of motion. We work in coordinates in which the background metric takes the form:

$$
G_{\mu \nu} d x^{\mu} d x^{\nu}=\frac{d \rho^{2}}{4 \rho^{2}}+\gamma_{i j} d x^{i} d x^{j} \quad \gamma_{i j} d x^{i} d x^{j}=-\frac{b^{2} d u^{2}}{\rho^{2}}+\frac{2 d u d v}{\rho}
$$

where $\rho=r^{2}$ with $r^{2}$ the coordinate appearing in equation (1.1). We use conventions where $N=1 /(2 \rho)$ and $\sqrt{-\gamma} \epsilon^{u v}=+1$. We then find that on the background:

$$
k=2 \quad k_{i j}=0 \quad \bar{k}_{i j}=b_{i j}
$$

with $b_{u u}=-b^{2} / \rho^{2}$ the only nonzero component of $b_{i j}$. From (5.16) we obtain:

$$
\pi_{i j}=-\gamma_{i j}
$$

Finally, from (5.7) it is trivial to see that:

$$
A_{i j}=0
$$

on the null warped background.

We now consider a small deformation $\delta G_{\mu \nu}=H_{\mu \nu}$ and we will work in a radial-axial gauge so that $H_{\rho \rho}=0$ and $H_{\rho i}=0$. We then define $h_{i j}=\rho \delta \gamma_{i j}=\rho H_{i j}$. We introduce the following notation for the first-order variation of the other fields:

$$
\begin{aligned}
\Pi_{i j} & =-\gamma_{i j}+\pi_{i j}[h] ; \\
k & =2+\kappa[h] ; \\
k_{i j} & =\kappa_{i j}[h] ; \\
\bar{k}_{i j} & =b_{i j}+\bar{\kappa}_{i j}[h] .
\end{aligned}
$$

Since we work at the linearized level, $\pi_{i j}, \kappa, \bar{\kappa}_{i j}$ and $\kappa_{i j}$ are all linear in $h_{i j}$. We mentioned below equation (5.10) that $k_{i j}$ and $\bar{k}_{i j}$ have a single independent component. To see what this implies for their fluctuations $\kappa_{i j}$ and $\bar{\kappa}_{i j}$ we linearize the equations (5.10) around the background (7.1). This results in:

$$
\begin{aligned}
\kappa_{u u} & =\frac{b^{4}}{4 \rho^{2}} \kappa_{v v}, & \kappa_{u v} & =-\frac{b^{2}}{2 \rho} \kappa_{v v}, \\
\bar{\kappa}_{u v} & =-\frac{b^{2}}{2 \rho^{2}} h_{v v}, & \bar{\kappa}_{v v} & =0,
\end{aligned}
$$

so the independent components of $\kappa_{i j}$ and $\bar{\kappa}_{i j}$ are $\kappa_{v v}$ and $\bar{\kappa}_{u u}$.

It is now convenient to express the fluctuations in momentum space as:

$$
h_{i j}(x)=\int \frac{d^{2} k}{(2 \pi)^{2}} e^{i k_{u} u+i k_{v} v} h_{i j}(k)
$$


Using the equations of motion (2.4) we then find at the linearized level:

$$
\begin{aligned}
0= & 6 k_{v} k_{u} \rho^{2} h_{u v}-3 k_{v}^{2} \rho^{2} h_{u u}-6 b^{2} h_{v v}+\left(-b^{2} k_{v}-3 k_{u} \rho\right)\left(-b^{2} k_{v}+k_{u} \rho\right) h_{v v} \\
& +2 \rho\left(\left(6-b^{2} k_{v}^{2}\right) \rho h_{u v}^{\prime}-k_{v}^{2} \rho^{2} h_{u u}^{\prime}+\left(k_{u}^{2} \rho^{2}+b^{2}\left(2+k_{v} k_{u} \rho\right)\right) h_{v v}^{\prime}-2 b^{2} \rho h_{v v}^{\prime \prime}\right) \\
0= & 2 k_{v} k_{u} \rho^{2}\left(b^{2} k_{v}+k_{u} \rho\right) h_{u v}+k_{v}^{2} \rho^{2}\left(-b^{2} k_{v}-k_{u} \rho\right) h_{u u} \\
& +\left(-b^{2} k_{v}-k_{u} \rho\right)\left(-6 b^{2}+k_{u}^{2} \rho^{2}\right) h_{v v}+4 \rho^{2}\left(\left(b^{2} k_{v}-3 k_{u} \rho\right) h_{u v}^{\prime}-b^{2} k_{u} h_{v v}^{\prime}\right. \\
& \left.+2 k_{v} \rho\left(2 h_{u u}^{\prime}+b^{2} h_{u v}^{\prime \prime}+\rho h_{u u}^{\prime \prime}\right)+b^{2}\left(b^{2} k_{v}+k_{u} \rho\right) h_{v v}^{\prime \prime}\right) \\
0= & -2 k_{u} k_{v}^{2} \rho^{2} h_{u v}+k_{v}^{3} \rho^{2} h_{u u}+k_{v}\left(6 b^{2}+k_{u}^{2} \rho^{2}\right) h_{v v} \\
& +4 \rho\left(-3 k_{v} \rho h_{u v}^{\prime}+\left(-b^{2} k_{v}+2 k_{u} \rho\right) h_{v v}^{\prime}-\rho\left(b^{2} k_{v}+2 k_{u} \rho\right) h_{v v}^{\prime \prime}\right) \\
0= & 2 b^{2} k_{v} k_{u} \rho^{2} h_{u v}-b^{2} k_{v}^{2} \rho^{2} h_{u u}-b^{2}\left(-b^{2}\left(6-b^{2} k_{v}^{2}\right)+2 b^{2} k_{v} k_{u} \rho+2 k_{u}^{2} \rho^{2}\right) h_{v v} \\
& -2 \rho\left(\rho\left(-b^{2}\left(2+b^{2} k_{v}^{2}\right)+k_{u}^{2} \rho^{2}\right) h_{u v}^{\prime}+k_{v} \rho^{2}\left(-b^{2} k_{v}-k_{u} \rho\right) h_{u u}^{\prime}\right. \\
& \left.-b^{2}\left(-k_{u}^{2} \rho^{2}-b^{2}\left(-2+k_{v} k_{u} \rho\right)\right) h_{v v}^{\prime}+4 \rho^{2}\left(2 b^{2} h_{u v}^{\prime \prime}+\rho\left(3 h_{u u}^{\prime \prime}+b^{2} h_{u v}^{(3)}+\rho h_{u u}^{(3)}\right)\right)\right) \\
0= & -b^{2}\left(-12-b^{2} k_{v}^{2}-k_{v} k_{u} \rho\right) h_{v v}-2 b^{2} k_{v}^{2} \rho^{2} h_{u v}^{\prime}-10 b^{2} \rho h_{v v}^{\prime} \\
& +\rho^{2}\left(-k_{v}^{2} \rho h_{u u}^{\prime}+k_{u}\left(2 b^{2} k_{v}+k_{u} \rho\right) h_{v v}^{\prime}+4\left(3 \rho h_{u v}^{\prime \prime}+b^{2} h_{v v}^{\prime \prime}-b^{2} \rho h_{v v}^{(3)}\right)\right) \\
0 & -\frac{b^{2} k_{v}^{2} h_{v v}}{\rho^{2}}+2 k_{v}^{2} h_{u v}^{\prime}-2 k_{v} k_{u} h_{v v}^{\prime}+8 \rho h_{v v}^{(3)}
\end{aligned}
$$

From the trace of the equation of motion (which is $R=-6$ ) we find:

$$
-2 k_{v} k_{u} \rho^{2} h_{u v}+k_{v}^{2} \rho^{2} h_{u u}+10 b^{2} h_{v v}+k_{u}^{2} \rho^{2} h_{v v}-4 \rho^{2} h_{u v}^{\prime}-8 b^{2} \rho h_{v v}^{\prime}+8 \rho^{3} h_{u v}^{\prime \prime}+4 b^{2} \rho^{2} h_{v v}^{\prime \prime}=0 .
$$

Let us consider the case where $k_{v}$ is nonzero. We can then use the trace equation to write $h_{u u}$ in terms of $h_{v v}$ and $h_{u v}$ and their derivatives. We then substitute this into (7.11) to find an expression for $h_{u v}^{\prime \prime}$ in terms of $h_{u v}, h_{u v}^{\prime}$ and $h_{v v}$ and its derivatives. Similarly, we can use (7.14) to solve for $h_{u v}^{\prime}$ in terms of $h_{v v}$ and its derivatives. Substituting now all these expressions into the first radial derivative of (7.9) results in the ordinary differential equation:

$$
\left(-b^{2} k_{v}^{2}-2 k_{u} k_{v} \rho\right) h_{v v}^{\prime \prime}+8 \rho h_{v v}^{(3)}+4 \rho^{2} h_{v v}^{(4)}=0 .
$$

Having solved this equation, one can obtain the other components from equation (7.14) and the trace equation. Note that

$$
h_{v v}^{\prime \prime}=0
$$

is a trivial solution of (7.16). Actually (7.17) is one of the equations obtained by linearizing three dimensional Einstein gravity. We will call the solution obtained by solving (7.17) the ' $\mathrm{T}$ ' solution and the solution obtained from the regular non-trivial solution of (7.16) the ' $\mathrm{X}$ ' solution. We will distinguish the two sets of solutions with superscripts $T$ and $X$.

The ' $\mathbf{T}$ ' solution. The first solution takes the form:

$$
\begin{aligned}
h_{u u}^{T} & =\frac{1}{\rho} h_{(-2) u u}+\tilde{h}_{(0) u u} \log (\rho)+h_{(0) u u}+\rho h_{(2) u u} \\
h_{u v}^{T} & =\frac{1}{\rho} h_{(-2) u v}+\tilde{h}_{(0) u v} \log (\rho)+h_{(0) u v}+\rho h_{(2) u v} \\
h_{v v}^{T} & =h_{(0) v v}+\rho h_{(2) v v}
\end{aligned}
$$


with:

$$
\begin{array}{rlrl}
k_{v}^{2} h_{(-2) u u} & =-b^{2}\left(k_{u} k_{v} h_{(0) v v}-4 h_{(2) v v}\right) & h_{(-2) u v} & =-\frac{1}{2} b^{2} h_{(0) v v} \\
k_{v} \tilde{h}_{(0) u u} & =b^{2} k_{u} h_{(2) v v} & \tilde{h}_{(0) u v} & =\frac{b^{2}}{2} h_{(2) v v} \\
h_{(2) u v} & =\frac{1}{4} \tilde{R}_{(0)} & k_{v} h_{(2) u u} & =\frac{1}{4} k_{u} \tilde{R}_{(0)} \\
k_{u} h_{(2) v v} & =\frac{1}{4} k_{v} \tilde{R}_{(0)} &
\end{array}
$$

and with

$$
\tilde{R}_{(0)}=k_{u}^{2} h_{(0) v v}-2 k_{v} k_{u} h_{(0) u v}+k_{v}^{2} h_{(0) u u}
$$

the linearized scalar curvature associated to a metric perturbation $\eta_{i j}+h_{(0) i j}$.

These modes are expected to correspond to switching on a source for the energymomentum tensor in the boundary theory. As already noted in the introduction, these solutions blow up faster at the boundary than the background metric. In earlier work, the boundary conditions used only allowed a subset of these solutions. For example, in the original paper of Son [1], an asymptotically Schrödinger metric of the type

$$
d s^{2}=\frac{d \rho^{2}}{4 \rho^{2}}+\frac{1}{\rho}\left(-\frac{b^{2} e^{-2 \Phi}}{\rho} d u^{2}+2 e^{-\Phi} d u\left(d v-a_{0} d v\right)\right)
$$

was proposed. (For convenience of comparison the notation here follows that of [1].) Here the functions $\Phi(\rho, u, v)$ and $a_{0}(\rho, u, v)$ were proposed to correspond to the energy and mass currents of the dual field theory, respectively, and were assumed to have a finite limit as $\rho \rightarrow 0$. Clearly the linearization of this ansatz does not match the solutions above: the $h_{v v}$ fluctuations have been switched off entirely. Constraining the asymptotics by not allowing for these fluctuations necessarily constrains or sets to zero certain of the operator sources in the field theory; related constraints were noticed in earlier discussions of renormalization for Schrödinger in [17]. Before analyzing the complete set of solutions of the linearized equations and setting up the holographic dictionary one cannot determine whether switching off $h_{v v}$ corresponds to switching off or constraining sources. While it would be consistent to set certain operator sources to zero, it is not consistent to constrain sources. It is also not consistent to set the vector fluctuation to zero, as proposed in [1]; in the analysis of the massive vector model in the next section we will see that the linearized field equations are coupled, and solutions involve both metric and vector fluctuations.

Let us also note that if the $h_{v v}$ fluctuations are switched off, then the remaining solution is asymptotically Schrödinger, in the sense that the fluctuations fall off at least as fast as the background as $\rho \rightarrow 0$. This connects with the discussion around (4.105): the operator $T_{u u}$ is irrelevant, whilst the other components of $T_{i j}$ are marginal or relevant, and (at $b=0$ ) $h_{(0) v v}$ acts as a source for $T_{u u}$. Switching on $h_{v v}$ therefore seems related to switching on the linearized source for $T_{u u}$, which would be expected to change the asymptotic structure of the background spacetime. 
The precise holographic interpretation of these metric modes is however rather subtle and will be discussed elsewhere. For now we will set all of these modes to zero, and focus on the second independent type of fluctuations:

The ' $\mathbf{X}$ ' solution. The second solution takes the form:

$h_{v v}^{X}=h_{(4-2 s) v v} \rho^{2-s}{ }_{1} F_{2}\left(1-s ; 2-2 s, 3-s ; \frac{1}{2} k_{v} k_{u} \rho\right)+h_{(2 s+2) v v} \rho^{s+1}{ }_{1} F_{2}\left(s ; 2 s, 2+s ; \frac{1}{2} k_{v} k_{u} \rho\right)$

with

$$
s=\frac{1}{2}+\frac{1}{2} \sqrt{1+b^{2} k_{v}^{2}}
$$

and with ${ }_{1} F_{2}$ the hypergeometric function. When we Taylor expand the hypergeometric functions around $\rho \rightarrow 0$ we find subleading terms whose form depends on whether $s \in$ $\{1,2,3, \ldots\}$ or not. Namely in the first case we find, just as for the scalar field, logarithmic terms in the expansion. We will not treat these cases here but note that one may deal with them in the same way as we did in section 6 . For non-integral $s$ the subleading terms become an ordinary power series:

$$
\begin{aligned}
h_{v v}= & h_{(4-2 s) v v} \rho^{2-s}\left(1+\alpha_{1}(s) k_{u} k_{v} \rho+\alpha_{2}(s)\left(k_{u} k_{v} \rho\right)^{2}+\ldots+\alpha_{n}(s)\left(k_{u} k_{v} \rho\right)^{n}+\ldots\right) \\
& +h_{(2 s+2) v v} \rho^{s+1}\left(1+\beta_{1}(s) k_{u} k_{v} \rho+\beta_{2}(s)\left(k_{u} k_{v} \rho\right)^{2}+\ldots+\beta_{n}(s)\left(k_{u} k_{v} \rho\right)^{n}+\ldots\right)
\end{aligned}
$$

where $\alpha_{n}(s)$ and $\beta_{n}(s)$ are rational functions of $s$. Their explicit form follows directly from the expansion of the hypergeometric function but it will not be needed here.

To compute a two point function we also need to identify which solution is regular throughout the bulk spacetime. To check regularity as $\rho \rightarrow \infty$ we use the asymptotic behavior:

$$
\begin{aligned}
\lim _{\rho \rightarrow \infty} \rho^{2-s}{ }_{1} F_{2}\left(1-s ; 2-2 s, 3-s ; \frac{1}{2} k_{v} k_{u} \rho\right) \\
\quad=\lim _{\rho \rightarrow \infty} \rho^{1 / 4} e^{2 \sqrt{k_{v} k_{u} \rho / 2}} \frac{1}{4 \sqrt{\pi}}(2-s) \Gamma(3-2 s)\left(\frac{k_{v} k_{u}}{2}\right)^{s-7 / 4}+\ldots
\end{aligned}
$$

and we find that the divergent pieces in $h_{v v}^{X}$ cancel only if we relate the two independent solutions as:

$$
h_{(2 s+2) v v}=\frac{(s-2) \Gamma(3-2 s)}{(s+1) \Gamma(1+2 s)}\left(\frac{k_{v} k_{u}}{2}\right)^{2 s-1} h_{(4-2 s) v v} .
$$

The solution for the other components $h_{u v}$ and $h_{u u}$ can be obtained by completely solving the linearized equations of motions. Their asymptotic behavior as $\rho \rightarrow 0$ takes the form:

$$
\begin{aligned}
& h_{u u}^{X}=\rho^{-s} h_{(-2 s) u u}(1+\ldots)+\rho^{s-1} h_{(2 s-2) u u}(1+\ldots) \\
& h_{u v}^{X}=\rho^{-s+1} h_{(2-2 s) u v}(1+\ldots)+\rho^{s} h_{(2 s) u v}(1+\ldots)
\end{aligned}
$$

with the leading coefficients given by:

$$
\begin{aligned}
& h_{(-2 s) u u}=\frac{16}{k_{v}^{4}} s(s-2)\left(s^{2}-1\right) h_{(4-2 s) v v} \\
& h_{(-2 s) u v}=-b^{2} \frac{(2 s-3)}{(2 s-2)} h_{(4-2 s) v v}
\end{aligned}
$$


and analogous formulas hold for the expression of $h_{(2 s-2) u u}$ and $h_{(2 s) u v}$ in terms of $h_{(2 s+2) v v}$. The ellipses in (7.30) represent subleading terms which have a similar form to those in $h_{v v}$ : in each case the ellipses represent an infinite power series of the form

$$
\sum_{n=1}^{\infty} \alpha_{n}(s)\left(k_{u} k_{v} \rho\right)^{n} .
$$

The coefficients $\alpha_{n}(s)$ are again rational functions of $s$ whose precise form is different for each of the occurrences of such a series in (7.30), but whose values are easily calculable starting from the expansion of $h_{v v}$.

Using the linearized version of (5.4) (with $N^{i}=0, N=1 /(2 \rho)$ ) and (5.9) we obtain that for this solution:

$$
\kappa_{v v}=2(s-2) h_{(4-2 s) v v} \rho^{-s+1}(1+\ldots)-(1+s) h_{(2 s+2) v v} \rho^{s}(1+\ldots)
$$

as well as:

$$
\begin{aligned}
\bar{\kappa}_{u u}= & \frac{8}{k_{v}^{4}} s^{2}(s-1)\left(s^{2}+5 s-14\right) h_{(4-2 s) v v} \rho^{-s-1}(1+\ldots) \\
& -\frac{4}{k_{v}^{4}}(s-1)^{2} s\left(s^{2}-7 s-8\right) h_{(2 s+2) v v} \rho^{s-2}(1+\ldots)
\end{aligned}
$$

and we recall that the other components of $\kappa_{i j}$ and $\bar{\kappa}_{i j}$ are given in (7.6) and (7.7). For $\pi_{i j}$ we linearize (5.16) and obtain:

$$
\begin{aligned}
\pi_{u u}= & -\frac{32}{3 k_{v}^{4}} s\left(6-11 s+14 s^{2}-13 s^{3}+4 s^{4}\right) h_{(4-2 s) v v} \rho^{-s-1}(1+\ldots) \\
& +\frac{16}{3 k_{v}^{4}}(s-1) s\left(4 s^{3}-3 s^{2}-s+6\right) h_{(2 s+2) v v} \rho^{s-2}(1+\ldots) \\
\pi_{u v}= & -\frac{4}{3 k_{v}^{2}} s\left(1+10 s-10 s^{2}+2 s^{3}\right) h_{(4-2 s) v v} \rho^{-s}(1+\ldots) \\
& -\frac{2}{3 k_{v}^{2}}(s-1)\left(-3-4 s+4 s^{2}+2 s^{3}\right) h_{(2 s+2) v v} \rho^{s-1}(1+\ldots) \\
\pi_{v v}= & \frac{2}{3}\left(2 s^{2}-4 s-3\right) h_{(4-2 s) v v} \rho^{-s+1}(1+\ldots) \\
& +\frac{1}{3}\left(2 s^{2}-5\right) h_{(2 s+2) v v} \rho^{s}(1+\ldots)
\end{aligned}
$$

Again the ellipses in the above five equations represent subleading terms of the form (7.32) with $\alpha_{n}(s)$ calculable rational functions of $s$ whose precise form depends on the quantity under consideration.

\subsection{On-shell action}

Let us begin with the on-shell action evaluated on the null warped background. We substitute the background values (7.2) and (7.3) in (5.13) to find that the on-shell action when evaluated on the background takes the form:

$$
S_{[0], \text { bare }}=\frac{1}{16 \pi G_{N}} \int d^{2} x \int_{\rho_{0}} d \rho \frac{2}{\rho^{2}}
$$


where the subscript [0] denotes the fact that this is the on-shell action to zeroth order in the perturbation and $\rho_{0}$ is a cutoff to regulate the action. As $\rho_{0} \rightarrow 0$ we find a divergence which is canceled by the counterterm:

$$
S_{[0], \mathrm{ct}}=-\frac{1}{8 \pi G_{N}} \int d^{2} x \sqrt{-\gamma}
$$

With this counterterm included, the combined action $S_{[0] \text {,bare }}+S_{[0], \text { ct }}$ is finite and actually vanishes as $\rho_{0} \rightarrow 0$.

We next consider the on-shell action evaluated to first order in the perturbations. We need to add the first-order variation of the on-shell action, given in (5.15), to the first-order variation of (7.37). This results in the following expression:

$$
S_{[1], \text { bare }}=\frac{1}{16 \pi G_{N}} \int d^{2} x \sqrt{-\gamma}\left(\frac{2}{3} k_{k}^{j} \delta \bar{k}_{j}^{k}+\left(-\Pi^{j k}-\gamma^{j k}+\frac{1}{6} A^{j k}\right) \delta \gamma_{j k}\right)
$$

where we added an extra sign with respect to (5.15) because the radial boundary is at the lower end of the $\rho$ integration and we have set $\mu=3$. Substituting the background values given in (7.2), (7.3) and (7.4) we find that all conjugate momenta vanish and and therefore $S_{[1]}$ vanishes as well.

With $S_{[0]}$ and $S_{[1]}$ vanishing, we conclude that the lowest-order term in the on-shell action is second order in the variations. From the variation of (7.38) we find that:

$$
S_{[2], \text { bare }}=\frac{1}{32 \pi G_{N}} \int d^{2} x \frac{1}{\rho}\left(\frac{2}{3} \kappa_{k}^{j} \bar{\kappa}_{j}^{k}-\left(\pi^{j k}+h^{j k}\right) h_{j k}\right) .
$$

Notice in particular that the second variation of the term $\int \sqrt{-\gamma} A^{i j} \delta \gamma_{i j}$ vanishes for our background, as can be directly seen from its definition (5.7).

Let us for now set the ' $\mathrm{T}$ ' modes to zero. We then substitute the expansions for the ' $\mathrm{X}$ ' modes given in subsection 7.1 in (7.39) to find its radial expansion:

$$
\begin{aligned}
S_{[2], \text { bare }}= & \frac{1}{32 \pi G_{N}} \int d^{2} k\left[\rho^{-2 s+1} \frac{32}{3 k_{v}^{4}}(-2+s)(-1+s) s^{2}\left(-12+7 s+s^{2}\right) h_{(4-2 s) v v}^{2}(1+\ldots)\right. \\
& \left.-\frac{32}{3 k_{v}^{4}}\left(-2-s+s^{2}\right)\left(-3 s+4 s^{2}-2 s^{3}+s^{4}\right) h_{(4-2 s) v v}(-k) h_{(2+2 s) v v}(k)+O(\rho)\right]
\end{aligned}
$$

where $h_{(4-2 s) v v}^{2}=h_{(4-2 s) v v}(-k) h_{(4-2 s) v v}(k)$. The dots again represent subleading terms which take the usual form (7.32) with certain rational coefficients $\alpha_{n}(s)$. These terms are divergent and need to be canceled with a suitable counterterm action. The $O(\rho)$ symbol represents the terms that vanish as the cutoff $\rho \rightarrow 0$.

To find the counterterms one may follow the usual procedure of holographic renormalization. However we discussed above that the current framework is not well adapted to discuss the ' $\mathrm{T}$ ' modes and we would therefore like to set these to zero. Unfortunately this introduces an ambiguity in the definition of the counterterm action because then all components of $h_{i j}$ and $\bar{\kappa}_{i j}$ essentially have the same component at leading order in their radial expansion. One may therefore replace for example $h_{u u}$ with $\bar{\kappa}_{u u}$ in a counterterm 
(and adjust the overall coefficient) without affecting the fact that it appropriately cancels a certain divergence. When we include the ' $\mathrm{T}$ ' modes however this ambiguity is lifted, since a counterterm which seemed suitable once the ' $\mathrm{T}$ ' modes are set to zero may actually induce extra divergences once they are nonzero. For this reason one cannot consistently perform the holographic renormalization without switching on the ' $\mathrm{T}$ ' modes as well and a more careful analysis of the counterterms will therefore be given elsewhere.

Nevertheless the structure of the divergences still allows us to obtain certain nontrivial results without performing the complete holographic renormalization. From a short analysis of the divergences and the possible counterterms one obtains that the renormalized on-shell action necessarily takes the same functional form as the term of order one in (7.40), albeit with a different normalization which can be an arbitrary function of $s$. We therefore write:

$$
S_{[2], \text { ren }}=S_{[2], \text { bare }}+S_{[2], \text { ct }}=\frac{1}{32 \pi G_{N}} \int d^{2} k \frac{c(s)}{k_{v}^{4}} h_{(4-2 s) v v}(-k) h_{(2+2 s) v v}(k)
$$

with an arbitrary function $c(s)$. The fact that $c(s)$ is undetermined as long as the holographic renormalization is not appropriately performed is similar to the well-known fact that for AlAdS spacetimes the correct normalization of the two-point function is obtained only once one properly holographically renormalizes the action. We repeat that we have set the ' $\mathrm{T}$ ' modes to zero by hand which is why they do not appear in (7.41).

Just as for the empty AdS background we will take the leading component of $\bar{\kappa}_{u u}$ to be the source of a dual operator $X_{v v}$. So if we rewrite (7.34) as:

$$
\bar{\kappa}_{u u}=\rho^{-s-1}\left(\bar{\kappa}_{(0) u u}(1+\ldots)+\bar{\kappa}_{(4 s-2) u u} \rho^{2 s-1}(1+\ldots)\right)
$$

with

$$
\begin{aligned}
\bar{\kappa}_{(0) u u} & =\frac{8}{k_{v}^{4}} s^{2}(s-1)\left(s^{2}+5 s-14\right) h_{(4-2 s) v v} \\
\bar{\kappa}_{(4 s-2) u u} & =-\frac{4}{k_{v}^{4}}(s-1)^{2} s\left(s^{2}-7 s-8\right) h_{(2 s+2) v v}
\end{aligned}
$$

then we should take $\bar{\kappa}_{(0) u u}$ to be the source for $X_{v v}$.

Using the above equation as well as (7.29) we can rewrite the renormalized action (7.41) in terms of $\bar{\kappa}_{(0) u u}$ :

$$
S_{[2], \text { ren }}=\frac{1}{32 \pi G_{N}} \int d^{2} k \tilde{c}(s) \bar{\kappa}_{(0) u u}(-k) k_{v}^{4}\left(k_{v} k_{u}\right)^{2 s-1} \bar{\kappa}_{(0) u u}(k)
$$

with undetermined normalization $\tilde{c}(s)$. The two-point function of $X_{v v}$ then takes the form:

$$
\left\langle X_{v v}(k) X_{v v}(-k)\right\rangle=-i \frac{\pi}{4 G_{N}} \tilde{c}(s) k_{v}^{4}\left(k_{v} k_{u}\right)^{2 s-1} .
$$

This is the behavior expected for the two-point function of an operator of weight $\Delta_{s}=2 s$ under the Schrödinger dilatation symmetry $\mathcal{D}$. As $b^{2} \rightarrow 0$, the correlation function also reduces to that of a $(3,1)$ operator in a CFT, as expected. 


\section{Linearized analysis for the massive vector model}

In this section we present the general linearized solution to the vector model equations of motion in three dimensions. We should note that in five dimensions gravity coupled to a massive vector is not by itself a consistent truncation of ten-dimensional supergravity; additional scalar fields need to be included [5]. The linearized equations of this consistent truncation do not coincide with those of the gravity plus vector model, but the generic features of the linearized solutions of this system are expected to be similar to those in the model analyzed here.

We choose a radial gauge such that:

$$
d s^{2}=\frac{d \rho^{2}}{4 \rho^{2}}+\left(\gamma_{i j}+\frac{h_{i j}}{\rho}\right) d x^{i} d x^{j}, \quad A_{\mu}=A_{\mu}^{b g}+\mathcal{A}_{\mu}
$$

where $\gamma_{i j}$ is the background metric (7.1), $A^{b g}=\frac{b}{\rho} d u$ is the background gauge field, and $h_{i j}$ and $\mathcal{A}_{\mu}$ parameterize small metric and gauge field perturbations. The linearized Einstein equations can then be written as:

$$
\begin{aligned}
& R_{i j}[h]+\operatorname{tr}\left(\gamma^{-1} h^{\prime}\right) \gamma_{i j}-\rho\left(2 h_{i j}^{\prime \prime}-4 b^{2} \rho^{-2} \delta_{(i}^{u} h_{j) v}^{\prime}+b^{2} \rho^{-2} \operatorname{tr}\left(\gamma^{-1} h^{\prime}\right) \delta_{i}^{u} \delta_{j}^{u}\right) \\
& =3 b^{2} \rho^{-2} h_{v v} \gamma_{i j}+b^{4} \rho^{-3} h_{v v} \delta_{i}^{u} \delta_{j}^{u}+4 b \rho^{-1} \mathcal{A}_{i} \delta_{j)}^{u}+4 b\left(\delta_{(i}^{u} \mathcal{F}_{j) \rho}-\mathcal{F}_{v \rho} \gamma_{i j}\right) \\
& \begin{aligned}
\partial_{i}\left(\operatorname{tr}\left(\gamma^{-1} h^{\prime}\right)\right) & -\gamma^{j k} \partial_{k} h_{i j}^{\prime}-\frac{1}{2} b^{2} \rho^{-2} \partial_{i} h_{u v v}+b^{2} \rho^{-2} \delta_{i}^{u} \partial_{k}\left(h_{v}^{k}-\frac{1}{2} \operatorname{tr}(h) \delta_{v}^{k}\right) \\
& =b \rho^{-1} \mathcal{F}_{i v}-4 b \rho^{-1} \mathcal{A}_{\rho} \delta_{i}^{u} ; \\
\frac{1}{2} b^{2} \partial_{\rho}\left(\rho^{-2} h_{v v}\right) & \\
= & \frac{1}{2} \operatorname{tr}\left(\gamma^{-1} h^{\prime \prime}\right) .
\end{aligned}
\end{aligned}
$$

Note also that $R_{i j}[h]-\frac{1}{2} \gamma_{i j} R[h]=0$, and $\mathcal{F}_{i j}=\partial_{i} \mathcal{A}_{j}-\partial_{j} \mathcal{A}_{i}$. The linearized vector field equations are

$$
\begin{aligned}
\partial_{i}\left(\gamma^{i j} \mathcal{F}_{j \rho}\right)-b \rho^{-2} \partial_{i}\left(h_{v}^{i}-\frac{1}{2}, \operatorname{tr}(h) \delta_{v}^{i}\right) & =\frac{4}{\rho} \mathcal{A}_{\rho} ; \\
\partial_{i}\left(\gamma^{i j} \gamma^{k l} \mathcal{F}_{j l}\right)+4 \partial_{\rho}\left(\rho \gamma^{i k} \mathcal{F}_{\rho i}\right)+4 b \rho^{-1} \partial_{\rho}\left(h_{v}^{k}-\frac{1}{2} \operatorname{tr}(h) \delta_{v}^{k}\right) & =\frac{4}{\rho} \gamma^{k i} \mathcal{A}_{i},
\end{aligned}
$$

whilst the linearized divergence equation is:

$$
\partial_{i}\left(\gamma^{i k} \mathcal{A}_{k}\right)+4 \rho \mathcal{A}_{\rho}^{\prime}-b \rho^{-1} \partial_{i}\left(h_{v}^{i}-\frac{1}{2} \operatorname{tr}(h) \delta_{v}^{i}\right)=0 .
$$

In these equations $\operatorname{tr}(h) \equiv \operatorname{tr}\left(\gamma^{-1} h\right)=b^{2} h_{v v} \rho^{-1}+2 h_{u v}$.

It is useful in solving these equations to carry out a Fourier transform with respect to the boundary coordinates $x^{i}$, by defining

$$
h_{i j}(x, \rho)=\int \frac{d^{2} k}{(2 \pi)^{2}} e^{i k_{u} u+i k_{v} v} \tilde{h}_{i j}(k, \rho), \quad \mathcal{A}_{\mu}(x, \rho)=\int \frac{d^{2} k}{(2 \pi)^{2}} e^{i k_{u} u+i k_{v} v} \tilde{\mathcal{A}}_{\mu}(k, \rho) .
$$


In order to keep notation uncluttered, we will drop the tilde in what follows. In solving the linearized equations of motion around the null warped background ${ }^{7}$ we find that the solutions again split into two independent sets, the ' $\mathrm{X}$ ' modes and ' $\mathrm{T}$ ' modes, as follows:

$$
h_{i j}(k, \rho)=h_{i j}^{T}(k, \rho)+h_{i j}^{X}(k, \rho), \quad \mathcal{A}_{\mu}(k, \rho)=\mathcal{A}_{\mu}^{T}(k, \rho)+\mathcal{A}_{\mu}^{X}(k, \rho) .
$$

As in the previous section, the ' $\mathrm{T}$ ' modes are associated with the dual stress energy tensor whilst the ' $\mathrm{X}$ ' modes are associated with the deforming vector operator.

The ' $T$ ' modes. This part of the solution can be written as:

$$
\begin{aligned}
h_{u u}^{T}(\rho, k) & =\frac{1}{\rho} h_{(-2) u u}-\tilde{h}_{(0) u u} \ln \rho+h_{(0) u u}+\rho h_{(2) u u} \\
h_{u v}^{T}(\rho, k) & =\frac{1}{\rho} h_{(-2) u v}-\tilde{h}_{(0) u v} \ln \rho+h_{(0) u v}+\rho h_{(2) u v} \\
h_{v v}^{T}(\rho, k) & =h_{(0) v v}+\rho h_{(2) v v} \\
\mathcal{A}_{\mu}(\rho, k) & =\frac{1}{\rho} \mathcal{A}_{(0) \mu}+\mathcal{A}_{(2) \mu}
\end{aligned}
$$

The various fields carrying $(n)$ subscripts are purely functions of $k^{i}$. The equations of motion relate the various functions as follows:

$$
\begin{aligned}
& \mathcal{A}_{(0) u}=-\frac{1}{2 b} h_{(-2) u u}, \quad \mathcal{A}_{(0) v}=-\frac{1}{b} h_{(-2) u v}, \quad \mathcal{A}_{(0) \rho}=-\frac{F}{4} \\
& A_{(2) i}=-\frac{\partial_{i} F}{4}, \quad A_{(2) \rho}=0, \quad F \equiv k_{u} A_{(0) v}-k_{v} A_{(0) u}
\end{aligned}
$$

Thus, the ' $\mathrm{T}$ ' modes of the gauge fields are completely determined in terms of $h_{(-2) i j}$ or vice versa. Now let us turn to the metric solution. The equations of motion give ${ }^{8}$

$$
h_{(-2) u v}=-\frac{b^{2}}{2} h_{(0) v v}, \quad 2 k_{u} k_{v} \tilde{h}_{(0) u v}=k_{v}^{2} \tilde{h}_{(0) u u}, \quad b^{2} h_{(2) v v}=2 \tilde{h}_{(0) u v} .
$$

The quantity $\tilde{R}_{(0)}$ has been defined in (7.23). Notice that the last relation implies a nonlocal relationship between the coefficients $h_{(-2) i j}$ and $h_{(0) i j}$. Finally, the remaining equations of motion give

$$
k_{v} h_{(2) u u}=k_{u} h_{(2) u v}, \quad k_{v} h_{(2) u v}=k_{u} h_{(2) v v}
$$

Combining the last equations we find that $\tilde{R}_{(0)}$ determines $h_{(2) i j}$ and $\tilde{h}_{(0) i j}$ as

$$
\begin{aligned}
& h_{(2) u u}=\frac{k_{u}}{4 k_{v}} \tilde{R}_{(0)}, \quad h_{(2) u v}=\frac{1}{4} \tilde{R}_{(0)}, \quad h_{(2) v v}=\frac{k_{v}}{4 k_{u}} \tilde{R}_{(0)} \\
& \tilde{h}_{(0) u u}=\frac{b^{2}}{4} \tilde{R}_{(0)}, \quad \quad \tilde{h}_{(0) u v}=\frac{b^{2} k_{v}}{8 k_{u}} \tilde{R}_{(0)}
\end{aligned}
$$

and a particular linear combination of the $h_{(-2) i j}$ as

$$
k_{u} k_{v} h_{(-2) u u}-2 k_{u}^{2} h_{(-2) u v}=b^{2} \tilde{R}_{(0)} .
$$

\footnotetext{
${ }^{7}$ We have used Mathematica to do this computation.

${ }^{8}$ Here the equations of motion have been solved order by order in $\rho$.
} 
Using the first relation in (8.12), we obtain

$$
k_{u} h_{(-2) u u}=b^{2}\left(k_{v} h_{(0) u u}-2 k_{u} h_{(0) u v}\right) .
$$

The ' $\mathrm{T}$ ' modes should correspond to the energy momentum tensor but are subject to the same subtleties as the ' $\mathrm{T}$ ' mode solutions of TMG.

\section{The ' $\mathrm{X}$ ' modes}

The second set of independent solutions are the ' $\mathrm{X}$ ' modes; one can view these as physical modes, since unlike the ' $\mathrm{T}$ ' modes they propagate in the bulk. We define the following function

$$
g(\rho)=\rho \mathcal{A}_{\rho}^{X},
$$

and substitute this into the equations of motion. We find

$$
\begin{aligned}
& \mathcal{A}_{u}^{X}=\frac{2 i k_{u} \rho}{k_{v}^{2} b^{2}}\left(k_{v} k_{u} g+2 g^{\prime}-2 \rho g^{\prime \prime}\right)+\frac{i}{k_{v} \rho}\left(4 g+3 k_{v} k_{u} \rho g-4 \rho^{2} g^{\prime \prime}\right)+\frac{i b^{2} k_{v} g}{\rho} \\
& \mathcal{A}_{v}^{X}=-\frac{2 i \rho}{k_{v} b^{2}}\left(k_{v} k_{u} g+2 g^{\prime}-2 \rho g^{\prime \prime}\right)-i k_{v} g, \quad \mathcal{A}_{\rho}^{X}=\frac{g}{\rho} .
\end{aligned}
$$

Only one metric component is nonzero for this part of the solution, namely

$$
h_{u u}^{X}=\frac{8 i}{b k_{v}^{3}}\left(2 k_{v} k_{u} g+4 g^{\prime}+k_{v} k_{u} \rho g^{\prime}-4 \rho g^{\prime \prime}-2 \rho^{2} g^{\prime \prime \prime}\right)+\frac{4 i b g^{\prime}}{k_{v}} .
$$

The equations of motion further require that $g(\rho)$ satisfy the fourth order equation:

$$
\begin{aligned}
\rho^{4} g^{(4)}+4 \rho^{3} g^{(3)} & -\left(\frac{1}{2} b^{2} k_{v}^{2} \rho^{2}+k_{v} k_{u} \rho^{3}\right) g^{\prime \prime} \\
- & k_{v} k_{u} \rho^{2} g^{\prime}+\left(\frac{1}{2} b^{2} k_{v}^{2}+\frac{b^{4} k_{v}^{4}}{16}+\frac{b^{2} k_{v}^{3} k_{u} \rho}{4}+\frac{k_{v}^{2} k_{u}^{2} \rho^{2}}{4}\right) g=0
\end{aligned}
$$

A feature of this equation is that it depends on $\rho$ only though the combination:

$$
x=\rho k_{v} k_{u} .
$$

Note in particular that this variable $x$ is invariant under the dilatation symmetry. In terms of the new variable, the equation (8.19) becomes:

$$
x^{4} g^{(4)}(x)+4 x^{3} g^{(3)}(x)-\left(2 \alpha x^{2}+x^{3}\right) g^{\prime \prime}(x)-x^{2} g^{\prime}(x)+\left(2 \alpha+\alpha^{2}+\alpha x+\frac{x^{2}}{4}\right) g(x)=0
$$

where

$$
\alpha \equiv \frac{b^{2} k_{v}^{2}}{4}
$$

and all primes now denote derivatives with respect to $x$. Unfortunately, the exact set of solutions of this equation has not been found. The equation becomes exactly solvable in terms of hypergeometric functions in the two limiting cases, (i) $x \ll a$ and (ii) $x \gg a$. In particular, the approximation in case (ii) leads to the same equation as in the undeformed theory, $b=0$, and we will discuss the exact solution for this case below. 
However, one can obtain the general asymptotic solution of (8.21). There are four independent set of such solutions which to leading order in the radial variable $x$ are:

$$
\begin{aligned}
g(x)= & x_{\left(2-\Delta_{1}\right)}(\alpha) x^{\frac{1}{2}-\frac{1}{2} \sqrt{1+b^{2} k_{v}^{2}}}+x_{\left(\Delta_{1}\right)}(\alpha) x^{\frac{1}{2}+\frac{1}{2} \sqrt{1+b^{2} k_{v}^{2}}} \\
& +x_{\left(2-\Delta_{2}\right)}(\alpha) x^{\frac{1}{2}-\frac{1}{2} \sqrt{9+b^{2} k_{v}^{2}}}+x_{\left(\Delta_{2}\right)}(\alpha) x^{\frac{1}{2}+\frac{1}{2} \sqrt{9+b^{2} k_{v}^{2}}} .
\end{aligned}
$$

where

$$
\Delta_{1}=1+\sqrt{1+b^{2} k_{v}^{2}}, \quad \Delta_{2}=1+\sqrt{9+b^{2} k_{v}^{2}} .
$$

Furthermore, the subleading terms in each independent solution are necessarily expanded as a power series in $x$, e.g. the first of the solutions behaves as:

$$
x^{\frac{1}{2}-\frac{1}{2} \sqrt{1+b^{2} k_{v}^{2}}}\left(x_{\left(2-\Delta_{1}\right)}(\alpha)+x_{\left(4-\Delta_{1}\right)}(\alpha) x+x_{\left(6-\Delta_{1}\right)}(\alpha) x^{2}+\cdots\right) .
$$

For generic values of the product $b^{2} k_{v}^{2}$ the dimensions $\left(\Delta_{1}, \Delta_{2}\right)$ are irrational, and the asymptotic expansion of each independent solution involves only radial powers. The notation used for $x_{(n)}$ indicates that in this case the coefficients scale as $x_{(n)} \rightarrow \lambda^{-n} x_{(n)}$ under the dilatation symmetry which acts as $\rho \rightarrow \lambda^{2} \rho, u \rightarrow \lambda^{2} u$. When the dimensions take rational values, however, logarithmic terms arise in the asymptotic expansion and in this case the coefficients do not all scale homogeneously under the dilatation symmetry.

From the form of the asymptotic solution, one would expect that the coefficients $x_{\left(2-\Delta_{i}\right)}$ are related to the sources for dual operators with dimensions $\Delta_{i}$, while $x_{\left(\Delta_{i}\right)}$ are associated with the corresponding vevs; we will explain this further below. Requiring smoothness ${ }^{9}$ of the solution for $g(\rho)$ in the interior of the spacetime should determine $x_{\left(\Delta_{i}\right)}$ in terms of $x_{\left(2-\Delta_{i}\right)}$ completely. Since these coefficients depends only on $\alpha$ the regularity conditions can depend only on the quantity $\alpha$, and thus will result in:

$$
x_{\left(\Delta_{i}\right)}=f_{i}(\alpha) x_{\left(2-\Delta_{i}\right)}
$$

where $f_{i}(\alpha)$ are functions of $\alpha$. This implies that the regular solution is of the form

$$
g(\rho)=\sum_{i=1}^{2} x_{\left(2-\Delta_{i}\right)} \rho^{1-\frac{1}{2} \Delta_{i}}\left(1+\cdots+\rho^{\Delta_{i}-1} f_{i}(\alpha) k_{v}^{\Delta_{i}-1} k_{u}^{\Delta_{i}-1}+\cdots\right)
$$

where the dots indicate subleading terms. In order to determine the explicit form of $f_{i}(\alpha)$ we would need to solve the full equation for $g(x)$, but fortunately the $k_{u}$-dependence is completely determined without knowing the full solution. Note that for rational values of the $\Delta_{i}$ this argument needs to be refined: logarithmic terms can arise in the asymptotic expansion and the coefficients $x_{\left(\Delta_{i}\right)}$ do not then scale homogeneously under dilatations. This happens for example at $b=0$ where the equation for $g(x)$ can be solved exactly: the two regular solutions are

$$
g(x)=g_{1} x K_{2}(\sqrt{2 x})+g_{1} x^{-1 / 2} K_{1}(\sqrt{2 x}),
$$

\footnotetext{
${ }^{9}$ Strictly speaking, the regular requirement should be imposed on the vector field itself, rather than on the function $g(\rho)$. However, the only feature we use in what follows is that regularity imposes two conditions on the four independent solutions.
} 
where $K_{n}(z)$ is the modified Bessel function of the second kind. Using the asymptotic expansion of these functions as $x \rightarrow 0$, we indeed find logarithmic terms; these relate to the terms logarithmic in momentum in the renormalized correlation functions, as expected for operators of integral dimension in a CFT.

It is also interesting to see how the $b^{2} \rightarrow 0$ limit is reached. Let us rewrite the leading terms in the expansion of $g(\rho)$ as:

$$
\begin{aligned}
g(\rho)= & i k_{v} g_{\left(2-\Delta_{1}\right)} \rho^{\frac{1}{2}-\frac{1}{2} \sqrt{1+b^{2} k_{v}^{2}}}+i b^{2} k_{v} g_{\left(2-\Delta_{2}\right)} \rho^{\frac{1}{2}-\frac{1}{2} \sqrt{9+b^{2} k_{v}^{2}}} \\
& +i b^{2} k_{v} g_{\left(\Delta_{1}\right)} \rho^{\frac{1}{2}+\frac{1}{2} \sqrt{1+b^{2} k_{v}^{2}}}+i k_{v} g_{\left(\Delta_{2}\right)} \rho^{\frac{1}{2}+\frac{1}{2} \sqrt{9+b^{2} k_{v}^{2}}}+\cdots
\end{aligned}
$$

where the ellipses denote the subleading terms and the $\left(x_{(1-\Delta)}, x_{(\Delta)}\right)$ have been rescaled relative to (8.23). The notation for $g_{(n)}$ again indicates that the coefficient scales as $g_{(n)} \rightarrow$ $\lambda^{-n} g_{(n)}$ under the dilatation symmetry which acts as $\rho \rightarrow \lambda^{2} \rho, u \rightarrow \lambda^{2} u$. Substituting these terms back into the vector field components we find:

$$
\begin{aligned}
& \mathcal{A}_{v}=\frac{4 g_{\left(2-\Delta_{1}\right)}}{b^{2}} \rho^{\frac{1}{2}-\frac{1}{2} \sqrt{1+b^{2} k_{v}^{2}}}\left(1-\sqrt{1+b^{2} k_{v}^{2}}\right)-4 g_{\left(2-\Delta_{2}\right)} \rho^{\frac{1}{2}-\frac{1}{2} \sqrt{9+b^{2} k_{v}^{2}}}\left(1+\sqrt{9+b^{2} k_{v}^{2}}\right) \\
& +2 g_{\left(\Delta_{1}\right)} \rho^{\frac{1}{2}+\frac{1}{2} \sqrt{1+b^{2} k_{v}^{2}}}\left(1+\sqrt{1+b^{2} k_{v}^{2}}\right)-\frac{g_{\left(\Delta_{2}\right)}}{b^{2}} \rho^{\frac{1}{2}+\frac{1}{2} \sqrt{9+b^{2} k_{v}^{2}}}\left(6-2 \sqrt{9+b^{2} k_{v}^{2}}\right)+\cdots ; \\
& \mathcal{A}_{u}=4 g_{\left(2-\Delta_{1}\right)} \rho^{-\frac{1}{2}-\frac{1}{2} \sqrt{1+b^{2} k_{v}^{2}}}-4 b^{2} g_{\left(2-\Delta_{2}\right)} \rho^{-\frac{1}{2}-\frac{1}{2} \sqrt{9+b^{2} k_{v}^{2}}} \\
& -6 b^{2} g_{\left(\Delta_{1}\right)} \rho^{-\frac{1}{2}+\frac{1}{2} \sqrt{1+b^{2} k_{v}^{2}}}+4 g_{\left(\Delta_{2}\right)} \rho^{-\frac{1}{2}+\frac{1}{2} \sqrt{9+b^{2} k_{v}^{2}}}+\cdots \text {. }
\end{aligned}
$$

Taking the limit of $b^{2} \rightarrow 0$ and retaining only the leading terms results in:

$$
\begin{aligned}
& \mathcal{A}_{v}=-16 g_{(-2)} \rho^{-1}-4 k_{v}^{2} g_{(0)}+4 g_{(2)} \rho+3 k_{v}^{2} g_{(4)} \rho^{2}+\cdots ; \\
& \mathcal{A}_{u}=4 g_{(0)} \rho^{-1}+4 g_{(4)} \rho+\cdots
\end{aligned}
$$

where we have used $\Delta_{1} \rightarrow 2$ and $\Delta_{2} \rightarrow 4$.

Let us next review the holographic correspondence at $b=0$, namely for linearized perturbations around the AdS background. The mass of the vector field is such that it corresponds to a vector operator of dimension three in the dual conformal field theory. Solving the linearized equations of motion for the vector around AdS results in an asymptotic expansion of the vector fluctuations of the form:

$$
\mathcal{A}_{i}=\frac{a_{i}(u, v)}{\rho}(1+\cdots)+\tilde{a}_{i}(u, v) \rho(1+\cdots),
$$

where $i=(u, v)$ and the ellipses denote subleading terms in $\rho$. Then $a_{i}$ is a source for the dual vector operator $X_{i}$ whilst its expectation value is related to the normalizable mode $\tilde{a}_{i}$. Comparing with (8.31) we note that:

$$
a_{v}=-16 g_{\left(2-\Delta_{2}\right)} ; \quad a_{u}=4 g_{\left(2-\Delta_{1}\right)} ; \quad \tilde{a}_{v}=4 g_{\left(\Delta_{1}\right)} ; \quad \tilde{a}_{u}=4 g_{\left(\Delta_{2}\right)},
$$

and thus the data $\left(g_{\left(2-\Delta_{1}\right)}, g_{\left(\Delta_{1}\right)}\right)$ is associated with the operator $X_{v}$ whilst the data $\left(g_{\left(2-\Delta_{2}\right)}, g_{\left(\Delta_{2}\right)}\right)$ is associated with the operator $X_{u}$. 


\subsection{Interpretation}

From the general linearized solution of the massive vector equations one can compute the two point functions of the dual stress energy tensor and of the deforming vector operator. This calculation however requires systematic holographic renormalization, which is rather complex and will be discussed elsewhere. Focusing on the ' $\mathrm{X}$ ' modes one can however make a number of interesting preliminary observations. Let us first recall what happens at $b=0$ in the AdS background; recall that the scaling dimensions with respect to the Schrödinger symmetry are such that $\Delta_{s}\left(X_{v}\right)=2$ and $\Delta_{s}\left(X_{u}\right)=4$, according to (4.62). From the asymptotics of the bulk vector field one sees that $a_{u}$ acts as a source for the $(2,1)$ operator $X_{v}$ whilst $a_{v}$ acts as a source for the $(1,2)$ operator $X_{u}$.

Working now to leading order in $b$, the field theory is deformed by a source for the operator $X_{v}$. The general arguments made in section 4 indicate that both operators, $X_{u}$ and $X_{v}$, would be expected to acquire lightcone momentum dependent anomalous dimensions, when $k_{v} \neq 0$. Looking at (8.30), if one continues to interpret $g_{\left(2-\Delta_{2}\right)}$ as a source for the deformed operator $X_{u}^{b}$ away from $b=0$, and similarly interprets $g_{\left(2-\Delta_{1}\right)}$ as a source for the deformed operator $X_{v}^{b}$, then

$$
\Delta_{s}\left(X_{v}^{b}\right)=\Delta_{1}=1+\sqrt{1+b^{2} k_{v}^{2}} ; \quad \Delta_{s}\left(X_{u}^{b}\right)=\Delta_{2}=1+\sqrt{9+b^{2} k_{v}^{2}} .
$$

These expressions are in agreement with (4.62) at $b=0$, and indicate that the anomalous dimensions at finite $b^{2}$ take this closed form. Given that the coefficients $g_{\left(\Delta_{1}\right)}$ and $g_{\left(\Delta_{2}\right)}$ have dilatation weights $\left(\Delta_{1}, \Delta_{2}\right)$ respectively, the vevs of the operators are expected to be of the form:

$$
\left\langle X_{u}\right\rangle \sim g_{\left(\Delta_{2}\right)} ; \quad\left\langle X_{v}\right\rangle \sim g_{\left(\Delta_{1}\right)},
$$

since for generic non-rational values of $\Delta_{i}$ no other terms in the asymptotic expansion of $g(\rho)$ have this dilatation weight. Using (8.27), we can immediately infer that

$$
\left\langle X_{u}(k) X_{u}(-k)\right\rangle \sim k_{u}^{\Delta_{2}-1} ; \quad\left\langle X_{v}(k) X_{v}(-k)\right\rangle \sim k_{u}^{\Delta_{1}-1},
$$

which is indeed of the form expected for operators of these scaling weights in a Schrödinger invariant theory. The $k_{v}$ dependent normalization can however only be determined using exact regular solutions of the linearized field equations together with holographic renormalization.

\section{Conclusions}

In this paper we have considered holography for $d+1$ dimensional Schrödinger backgrounds and argued that they are dual to $d$ dimensional theories on Minkowski spacetime obtained by deforming conformal field theories by irrelevant operators, which are however exactly marginal from the perspective of the non-relativistic conformal group. In other words, they describe a continuous deformation from a relativistic fixed point to a non-relativistic fixed point. On the field theory side, we used conformal perturbation theory to study this system (so the undeformed CFT can be either weakly or strongly coupled) and showed that 
the deforming operator is indeed exactly marginal. We also studied how the dimensions of operators change, to leading order in the deformation parameter $b^{2}$, when we deform the theory. These results are in agreement with the results obtained using the gravity dual, when linearized in $b^{2}$. The gravitational result, however, is valid for any $b^{2}$ and resums the corrections into a closed, squared root form. An important result is that the boundary counterterms obtained by holographic renormalization are are non-local in the lightcone $v$ direction, implying that the boundary theory is not a local QFT.

Working at the linearized level in the metric sector, we saw that the general solution of the equations of motion blows up faster at the boundary than the background Schrödinger solution. This was not unexpected, since the linearized solutions correspond to operators that are irrelevant with respect to the non-relativistic scaling symmetry, and therefore a finite source for this operator would be expected to change the asymptotic structure of the spacetime. Similarly components of the stress energy tensor are also irrelevant with respect the Schrödinger dilatation symmetry. This feature is responsible for many subtleties in setting up holographic renormalization for Schrödinger, and needs to be understood better before generalizing the analysis to the non-linear level. The conserved stress energy tensor of the dual theory couples to the vielbein, rather than the metric, and hence the correct holographic setup is to fix boundary conditions for the vielbein, rather than the metric. The holographic dictionary for the gravitational sector is thus rather technically involved, even at the linearized level, and it will be discussed elsewhere.

Throughout this paper we have noted a number of interesting open questions. The Schrödinger invariant theory should admit a realization as a null dipole theory; for each "ordinary" CFT one would obtain the null dipole theory by replacing ordinary products with null dipole products. Such a realization should allow one to compute the scaling dimensions $\Delta_{s}$ in the deformed theory in terms of the conformal dimensions $\Delta$ in the ordinary CFT. Moreover the analog of the Seiberg-Witten map [32] between the null dipole and ordinary theories should allow one to understand the detailed structure of the counterterms. In particular, we noticed that in the holographic realization the boundary counterterms for a scalar field in Schrödinger were simply related to those of a scalar field in AdS, and this may well be a consequence of an underlying null dipole structure. It would also be interesting to understand the counterterms better from the field theory perspective; working perturbatively in $b^{2}$ we expect a series of counterterms involving increasing numbers of lightcone derivatives to be induced. This series of terms would then be expected to resum into the structure obtained in the null dipole theory.

\section{Acknowledgments}

We would like to thank A. Adams, A. Dabholkar, A. Sen and A. Strominger for discussions. KS and MMT would especially like to thank Allan Adams for stimulating their interest in Schrödinger holography. KS and MMT would like to thank the Aspen Center of Physics for hospitality during the initial stages of this work and the Simons Workshop in Mathematics and Physics 2010 for hospitality during the final stages of this work. This work is part of the research program of the 'Stichting voor Fundamenteel Onderzoek der Materie (FOM)', 
which is financially supported by the 'Nederlandse Organisatie voor Wetenschappelijk Onderzoek (NWO)'. The authors acknowledge support from NWO, KS via a Vici grant, MMT via the Vidi grant "Holography, duality and time dependence in string theory" and BvR via an NWO Spinoza grant.

Open Access. This article is distributed under the terms of the Creative Commons Attribution Noncommercial License which permits any noncommercial use, distribution, and reproduction in any medium, provided the original author(s) and source are credited.

\section{References}

[1] D.T. Son, Toward an AdS/cold atoms correspondence: a geometric realization of the Schroedinger symmetry, Phys. Rev. D 78 (2008) 046003 [arXiv:0804.3972] [SPIRES].

[2] K. Balasubramanian and J. McGreevy, Gravity duals for non-relativistic CFTs, Phys. Rev. Lett. 101 (2008) 061601 [arXiv:0804.4053] [SPIRES].

[3] C. Duval, G.W. Gibbons and P. Horvathy, Celestial mechanics, conformal structures and gravitational waves, Phys. Rev. D 43 (1991) 3907 [hep-th/0512188] [SPIRES].

[4] C. Duval, M. Hassaine and P.A. Horvathy, The geometry of Schrödinger symmetry in gravity background/non-relativistic CFT, Annals Phys. 324 (2009) 1158 [arXiv:0809.3128] [SPIRES].

[5] J. Maldacena, D. Martelli and Y. Tachikawa, Comments on string theory backgrounds with non-relativistic conformal symmetry, JHEP 10 (2008) 072 [arXiv:0807.1100] [SPIRES].

[6] K. Balasubramanian and J. McGreevy, The particle number in Galilean holography, JHEP 01 (2011) 137 [arXiv:1007.2184] [SPIRES].

[7] G. Burdet, M. Perrin and P. Sorba, About the non-relativistic structure of the conformal algebra, Commun. Math. Phys, 34 (1973) 85.

[8] E. Barnes, D. Vaman and C. Wu, Holographic real-time non-relativistic correlators at zero and finite temperature, Phys. Rev. D 82 (2010) 125042 [arXiv:1007.1644] [SPIRES].

[9] M. Henkel, Schrödinger invariance in strongly anisotropic critical systems, J. Stat. Phys. 75 (1994) 1023 [hep-th/9310081] [SPIRES].

[10] K. Skenderis, M. Taylor and B.C. van Rees, Topologically massive gravity and the AdS/CFT correspondence, JHEP 09 (2009) 045 [arXiv:0906.4926] [SPIRES].

[11] K. Skenderis, M. Taylor and B.C. van Rees, AdS boundary conditions and the topologically massive gravity/CFT correspondence, arXiv:0909.5617 [SPIRES].

[12] K. Skenderis, Lecture notes on holographic renormalization, Class. Quant. Grav. 19 (2002) 5849 [hep-th/0209067] [SPIRES].

[13] S.S. Gubser, I.R. Klebanov and A.M. Polyakov, Gauge theory correlators from non-critical string theory, Phys. Lett. B 428 (1998) 105 [hep-th/9802109] [SPIRES].

[14] E. Witten, Anti-de Sitter space and holography, Adv. Theor. Math. Phys. 2 (1998) 253 [hep-th/9802150] [SPIRES].

[15] W.D. Goldberger, AdS/CFT duality for non-relativistic field theory, JHEP 03 (2009) 069 [arXiv:0806.2867] [SPIRES]. 
[16] P. Hořava and C.M. Melby-Thompson, Anisotropic conformal infinity, arXiv:0909.3841 [SPIRES].

[17] C.P. Herzog, M. Rangamani and S.F. Ross, Heating up galilean holography, JHEP 11 (2008) 080 [arXiv:0807.1099] [SPIRES].

[18] S.F. Ross and O. Saremi, Holographic stress tensor for non-relativistic theories, JHEP 09 (2009) 009 [arXiv: 0907.1846] [SPIRES].

[19] A. Adams, K. Balasubramanian and J. McGreevy, Hot spacetimes for cold atoms, JHEP 11 (2008) 059 [arXiv:0807.1111] [SPIRES].

[20] A. Bergman and O.J. Ganor, Dipoles, twists and noncommutative gauge theory, JHEP 10 (2000) 018 [hep-th/0008030] [SPIRES].

[21] K. Dasgupta, O.J. Ganor and G. Rajesh, Vector deformations of $N=4$ super-Yang-Mills theory, pinned branes and arched strings, JHEP 04 (2001) 034 [hep-th/0010072] [SPIRES].

[22] A. Bergman, K. Dasgupta, O.J. Ganor, J.L. Karczmarek and G. Rajesh, Nonlocal field theories and their gravity duals, Phys. Rev. D 65 (2002) 066005 [hep-th/0103090] [SPIRES].

[23] D. Anninos, W. Li, M. Padi, W. Song and A. Strominger, Warped AdS $S_{3}$ black holes, JHEP 03 (2009) 130 [arXiv:0807.3040] [SPIRES].

[24] M. Blau, J. Hartong and B. Rollier, Geometry of Schrödinger space-times, global coordinates and harmonic trapping, JHEP 07 (2009) 027 [arXiv: 0904.3304] [SPIRES].

[25] S.A. Hartnoll and K. Yoshida, Families of IIB duals for nonrelativistic CFTs, JHEP 12 (2008) 071 [arXiv:0810.0298] [SPIRES].

[26] J.P. Gauntlett, S. Kim, O. Varela and D. Waldram, Consistent supersymmetric Kaluza-Klein truncations with massive modes, JHEP 04 (2009) 102 [arXiv:0901.0676] [SPIRES].

[27] A. Donos and J.P. Gauntlett, Supersymmetric solutions for non-relativistic holography, JHEP 03 (2009) 138 [arXiv:0901.0818] [SPIRES].

[28] E. O Colgain and H. Yavartanoo, NR CFT 3 duals in M-theory, JHEP 09 (2009) 002 [arXiv:0904.0588] [SPIRES].

[29] N. Bobev, A. Kundu and K. Pilch, Supersymmetric IIB solutions with Schrödinger symmetry, JHEP 07 (2009) 107 [arXiv:0905.0673] [SPIRES].

[30] A. Donos and J.P. Gauntlett, Solutions of type IIB and D=11 supergravity with Schrödinger(z) symmetry, JHEP 07 (2009) 042 [arXiv: 0905.1098] [SPIRES].

[31] A. Donos and J.P. Gauntlett, Schrödinger invariant solutions of type IIB with enhanced supersymmetry, JHEP 10 (2009) 073 [arXiv:0907.1761] [SPIRES].

[32] N. Seiberg and E. Witten, String theory and noncommutative geometry, JHEP 09 (1999) 032 [hep-th/9908142] [SPIRES].

[33] S. Minwalla, M. Van Raamsdonk and N. Seiberg, Noncommutative perturbative dynamics, JHEP 02 (2000) 020 [hep-th/9912072] [SPIRES].

[34] O. Aharony, J. Gomis and T. Mehen, On theories with light-like noncommutativity, JHEP 09 (2000) 023 [hep-th/0006236] [SPIRES].

[35] S. de Haro, S.N. Solodukhin and K. Skenderis, Holographic reconstruction of spacetime and renormalization in the AdS/CFT correspondence, Commun. Math. Phys. 217 (2001) 595 [hep-th/0002230] [SPIRES]. 
[36] M. Bianchi, D.Z. Freedman and K. Skenderis, Holographic renormalization, Nucl. Phys. B 631 (2002) 159 [hep-th/0112119] [SPIRES].

[37] I. Papadimitriou and K. Skenderis, AdS/CFT correspondence and geometry, hep-th/0404176 [SPIRES].

[38] I. Papadimitriou and K. Skenderis, Correlation functions in holographic RG flows, JHEP 10 (2004) 075 [hep-th/0407071] [SPIRES].

[39] D.Z. Freedman, K. Johnson and J.I. Latorre, Differential regularization and renormalization: a new method of calculation in quantum field theory, Nucl. Phys. B 371 (1992) 353 [SPIRES].

[40] A. Karch, A. O'Bannon and K. Skenderis, Holographic renormalization of probe D-branes in AdS/CFT, JHEP 04 (2006) 015 [hep-th/0512125] [SPIRES].

[41] R.N.C. Costa and M. Taylor, Holography for chiral scale-invariant models, arXiv:1010.4800 [SPIRES].

[42] S. Olmez, O. Sarioglu and B. Tekin, Mass and angular momentum of asymptotically AdS or flat solutions in the topologically massive gravity, Class. Quant. Grav. 22 (2005) 4355 [gr-qc/0507003] [SPIRES].

[43] D.D.K. Chow, C.N. Pope and E. Sezgin, Classification of solutions in topologically massive gravity, Class. Quant. Grav. 27 (2010) 105001 [arXiv:0906.3559] [SPIRES].

[44] D. Anninos, G. Compere, S. de Buyl, S. Detournay and M. Guica, The curious case of null warped space, JHEP 11 (2010) 119 [arXiv:1005.4072] [SPIRES].

[45] K. Skenderis and M. Taylor, Kaluza-Klein holography, JHEP 05 (2006) 057 [hep-th/0603016] [SPIRES].

[46] K. Skenderis and M. Taylor, Holographic coulomb branch vevs, JHEP 08 (2006) 001 [hep-th/0604169] [SPIRES].

[47] G. Arutyunov and S. Frolov, Some cubic couplings in type IIB supergravity on $A d S_{5} \times S^{5}$ and three-point functions in $S Y M(4)$ at large-N, Phys. Rev. D 61 (2000) 064009 [hep-th/9907085] [SPIRES].

[48] S. Deser and X. Xiang, Canonical formulations of full nonlinear topologically massive gravity, Phys. Lett. B 263 (1991) 39 [SPIRES].

[49] I.L. Buchbinder, S.L. Lyahovich and V.A. Krychtin, Canonical quantization of topologically massive gravity, Class. Quant. Grav. 10 (1993) 2083 [SPIRES].

[50] K. Hotta, Y. Hyakutake, T. Kubota and H. Tanida, Brown-Henneaux's canonical approach to topologically massive gravity, JHEP 07 (2008) 066 [arXiv:0805.2005] [SPIRES].

[51] M. Blau, J. Hartong and B. Rollier, Geometry of Schrödinger space-times II: particle and field probes of the causal structure, JHEP 07 (2010) 069 [arXiv: 1005.0760] [SPIRES].

[52] K. Skenderis and B.C. van Rees, Real-time gauge/gravity duality, Phys. Rev. Lett. 101 (2008) 081601 [arXiv:0805.0150] [SPIRES].

[53] K. Skenderis and B.C. van Rees, Real-time gauge/gravity duality: prescription, renormalization and examples, JHEP 05 (2009) 085 [arXiv:0812.2909] [SPIRES].

[54] R.G. Leigh and N.N. Hoang, Real-time correlators and non-relativistic holography, JHEP 11 (2009) 010 [arXiv:0904.4270] [SPIRES]. 
[55] C.A. Fuertes and S. Moroz, Correlation functions in the non-relativistic AdS/CFT correspondence, Phys. Rev. D 79 (2009) 106004 [arXiv:0903.1844] [SPIRES].

[56] A. Volovich and C. Wen, Correlation functions in non-relativistic holography, JHEP 05 (2009) 087 [arXiv:0903.2455] [SPIRES].

[57] B. Chen and Z.-b. Xu, Quasi-normal modes of warped black holes and warped AdS/CFT correspondence, JHEP 11 (2009) 091 [arXiv:0908.0057] [SPIRES].

[58] B. Chen, B. Ning and Z.-b. Xu, Real-time correlators in warped AdS/CFT correspondence, JHEP 02 (2010) 031 [arXiv:0911.0167] [SPIRES].

[59] D.Z. Freedman, S.D. Mathur, A. Matusis and L. Rastelli, Correlation functions in the CFT $(d) / A d S(d+1)$ correspondence, Nucl. Phys. B 546 (1999) 96 [hep-th/9804058] [SPIRES].

[60] I. Papadimitriou and K. Skenderis, Thermodynamics of asymptotically locally AdS spacetimes, JHEP 08 (2005) 004 [hep-th/0505190] [SPIRES].

[61] I. Papadimitriou, Holographic renormalization as a canonical transformation, arXiv: 1007.4592 [SPIRES]. 